\title{
An Evidence-Based Multidisciplinary Approach Focused at Creating Algorithms for Targeted Therapy of BSIs, cUTIs, and clAls Caused by Enterobacterales in Critically III Adult Patients
}

\author{
Milo Gatti ${ }^{1,2}$ \\ Bruno Viaggi ${ }^{3}$ \\ Gian Maria Rossolini (iD) ${ }^{4-6}$ \\ Federico Pea (iD) ${ }^{1,2}$ \\ Pierluigi Viale ${ }^{1,7}$ \\ 'Department of Medical and Surgical \\ Sciences, Alma Mater Studiorum, \\ University of Bologna, Bologna, Italy; \\ ${ }^{2}$ SSD Clinical Pharmacology, IRCCS \\ Azienda Ospedaliero Universitaria \\ Sant'Orsola, Bologna, Italy; \\ ${ }^{3}$ Neurointensive Care Unit, Department \\ of Anesthesiology, Careggi, University \\ Hospital, Florence, Italy; ${ }^{4}$ Department of \\ Experimental and Clinical Medicine, \\ University of Florence, Florence, Italy; \\ ${ }^{5}$ Microbiology and Virology Unit, \\ Florence Careggi University Hospital, \\ Florence, Italy; 'IRCCS Fondazione Don \\ Carlo Gnocchi, Florence, Italy; \\ ${ }^{7}$ Infectious Diseases Unit, IRCCS Azienda \\ Ospedaliero Universitaria Sant'Orsola, \\ Bologna, Italy
}

\begin{abstract}
Prompt implementation of appropriate targeted antibiotic therapy represents a valuable approach in improving clinical and ecological outcome in critically septic patients. This multidisciplinary opinion article focused at developing evidence-based algorithms for targeted antibiotic therapy of bloodstream (BSIs), complicated urinary tract (cUTIs), and complicated intrabdominal infections (cIAIs) caused by Enterobacterales. The aim was to provide a guidance for intensive care physicians either in appropriately placing novel antibiotics or in considering strategies for sparing the broadest-spectrum antibiotics. A multidisciplinary team of experts (one intensive care physician, one infectious disease consultant, one clinical microbiologist and one MD clinical pharmacologist), performed several rounds of assessment to reach agreement in developing six different algorithms according to the susceptibility pattern (one each for multi-susceptible, extended-spectrum beta-lactamase-producing, AmpC beta-lactamase-producing, Klebsiella pneumoniae carbapenemase (KPC)-producing, OXA-48-producing, and Metallo-beta-lactamase (MBL)-producing Enterobacterales). Whenever multiple therapeutic options were feasible, a hierarchical scale was established. Recommendations on antibiotic dosing optimization were also provided. In order to retrieve evidence-based support for the therapeutic choices proposed in the algorithms, a comprehensive literature search was performed by a researcher on PubMedMEDLINE from inception until March 2021. Quality and strength of evidence was established according to a hierarchical scale of the study design. Only articles published in English were included. It is expected that these algorithms, by allowing prompt revision of antibiotic regimens whenever feasible, appropriate place in therapy of novel beta-lactams, implementation of strategies for sparing the broadest-spectrum antibiotics, and pharmacokinetic/pharmacodynamic optimization of antibiotic dosing regimens, may be helpful either in improving clinical outcome or in containing the spread of antimicrobial resistance.
\end{abstract}

Keywords: critically ill patients, targeted antibiotic therapy, antimicrobial stewardship, Enterobacterales, multidisciplinary taskforce, PK/PD dosing optimization

\section{Introduction}

Sepsis is a common occurrence in patients admitted to intensive care unit (ICU), accounting for high mortality and massive antibiotic consumption. ${ }^{1-3}$ Bloodstream infections (BSIs), complicated intra abdominal infections (cIAIs) and complicated urinary tract infections (cUTIs) are second only to pneumonia as sources of infections among ICU patients. ${ }^{4,5}$ Enterobacterales account for the most frequently
Department of Medical and Surgical

Sciences, Alma Mater Studiorum,

University of Bologna, Via Massarenti, 9,

Bologna, 40138 , Italy

Tel +39051 2143199

Email federico.pea@unibo.it 
isolated pathogens. ${ }^{5,6}$ Beta-lactams represent mainstay of treatment, and may have different roles according to the susceptibility pattern of clinical isolates. Previous antibiotic use, colonization by or ICU acquisition of MDREnterobacterales, prolonged hospitalisation, severity of acute illness are the major determinants of risk for developing infections caused by multidrug-resistant (MDR) Enterobacterales. ${ }^{7-9}$ Six different susceptibility patterns to beta-lactams may be identified among Enterobacterales: multi-susceptible, extended-spectrum beta-lactamase (ESBL)-producing, AmpC beta-lactamase (AmpC)-producing, Klebsiella pneumoniae carbapenemase (KPC)-producing, OXA-48-producing, and metallobeta-lactamase (MBL)- producing Enterobacterales. ${ }^{7-9}$

Early and appropriate antimicrobial treatment represents a cornerstone in the management of critically septic patient. ${ }^{10,11}$ The Surviving Sepsis Campaign guidelines recommend prompt implementation of targeted antibiotic therapy once that pathogen has been identified and antimicrobial susceptibility has been tested. ${ }^{12}$ A multidisciplinary team composed of the intensive care physician, the infectious disease consultant, the clinical microbiologist, and the MD clinical pharmacologist (Figure 1), could be helpful to pursuit this aim. Prompt implementation of appropriate definitive therapy according to the "antimicrobial puzzle" concepts ${ }^{13}$ could play a key role in improving clinical and ecological outcome in critical settings. ${ }^{14,15}$

This multidisciplinary opinion article aims to develop evidence-based algorithms for targeted antibiotic therapy of BSIs, cIAIs, and cUTIs caused by Enterobacterales in critically ill adult patients.
The aim was to provide a useful guidance for intensive care physicians either in appropriately placing novel antimicrobial agents in lack of definitive evidence or in considering antimicrobial stewardship strategies for sparing the broadest-spectrum antibiotics.

\section{Materials and Methods}

A multidisciplinary team composed by one intensive care physician (B.V.), one infectious disease consultant (P.V.), one clinical microbiologist (G.M.R.), and one MD clinical pharmacologist (F.P.) met virtually on several occasions to reach agreement in developing algorithms and specific recommendations for targeted antimicrobial therapy of BSIs, cIAIs, and cUTIs caused by Enterobacterales in ICU critically ill patients. The definitive agreement for each therapeutic algorithm was reached by the multidisciplinary team after thoroughly discussion based on specific long-standing experience and on the specific expertise of each single member. The rationale for considering common algorithms for these infection sites is based on the fact that cIAIs and cUTIs were investigated together in the last pivotal trials concerning novel antibiotics. ${ }^{16}$ Additionally, bacteraemic and non-bacteraemic cUTIs and cIAIs are commonly considered as relatively benign infection sources showing no high-inoculum effect, differently from that occurs in severe nosocomial pneumonia. ${ }^{17,18}$ Consequently, we believe that algorithms for targeted therapy of infection-related ventilator associated complications (IVACs) must be considered apart. Six different scenarios were structured according to the pattern of antibiotic susceptibility of the pathogens and/or of the genotype of

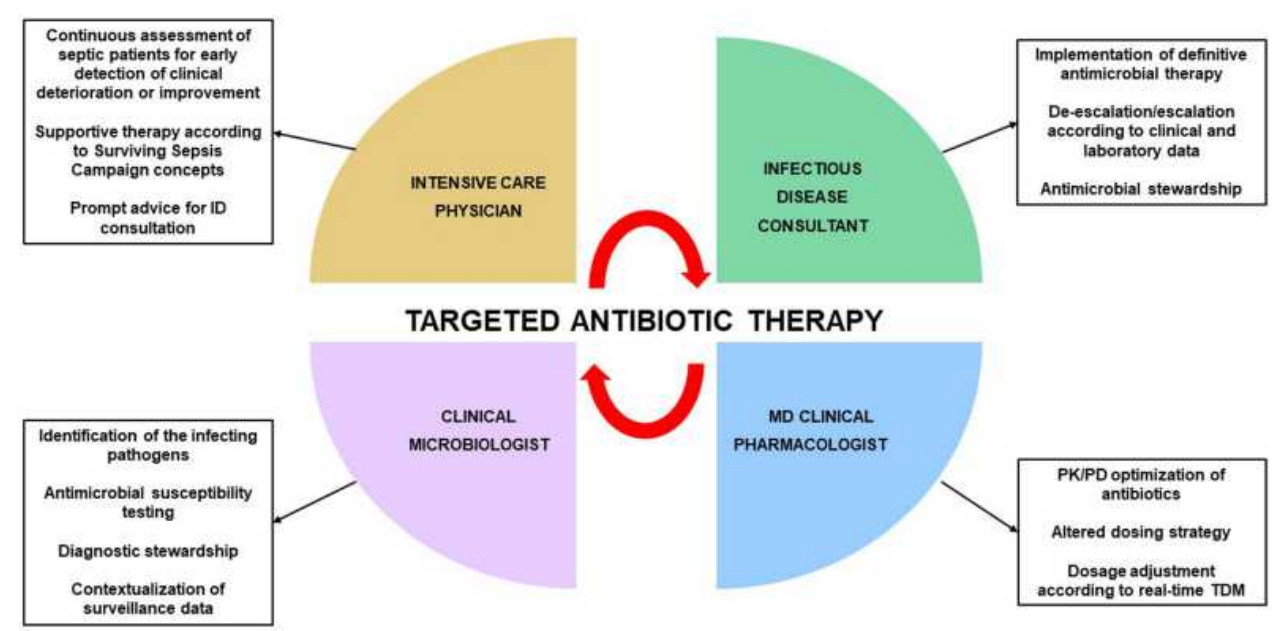

Figure I Features of multidisciplinary taskforce involved in implementation of targeted antimicrobial therapy in critically ill patients.

Abbreviations: ID, infectious disease; PCR, polymerase chain reaction; PK/PD; pharmacokinetic/pharmacodynamic; TDM, therapeutic drug monitoring. 
resistance. Whenever multiple therapeutic options were feasible, a hierarchical scale was established. Recommendations on antibiotic dosing optimization were also provided.

Scientific evidence supporting the specific choices included in the algorithms was retrieved by means of a literature search conducted by a researcher (M.G.) on PubMed-MEDLINE (from inception until March 2021). Key terms for search included selected antibiotics, site of infections, and genotype of resistance and/or pattern of susceptibility of bacterial pathogens. Quality of evidence was established according to a hierarchical scale of the study design, as reported in the evidence pyramid: ${ }^{19}$ randomized controlled trials (RCTs); prospective observational studies; retrospective observational studies; case series; case reports; in vitro studies. International guidelines issued by the Infectious Disease Society of America and/or by the European Society of Clinical Microbiology and Infectious Diseases, systematic reviews and meta-analyses were also consulted. Consistence between retrieved studies was also considered, by assessing the concordance in clinical outcome of the included studies at each level of the evidence pyramid. Only articles published in English were included, and search was focused mainly on the last ten years in order to provide an up-to-date overview on the scientific evidence that may support the therapeutic algorithms.

\section{Targeted Treatment of BSIs, cUTIs, and clAls Caused by Enterobacterales in Critically III Adult Patients}

Six different algorithms for targeted treatment of BSIs, cUTIs, cIAIs are depicted in Figure 2, one each for infections caused by multi-susceptible, ESBL-, AmpC-, KPC-, OXA-48-, and MBL-producing Enterobacterales.

\section{Multi-Susceptible Enterobacterales}

Recommendations are depicted in Figure 2, panel A.1. Ampicillin-sulbactam [3g q6h over $6 \mathrm{~h}$ by continuous infusion $(\mathrm{CI})$ after $3 \mathrm{~g}$ loading dose [LD]] or ceftriaxone $(2 \mathrm{~g}$ q24h) are recommended for BSIs, cUTIs, and cIAIs caused by multi-susceptible Enterobacterales. Evidences supporting these choices are summarized in Table 1. Both the European $^{20}$ and the American ${ }^{21}$ guidelines recommended the use of ampicillin-sulbactam or ceftriaxone for the management of mild-to-moderate cIAIs/peritonitis. In regard to ampicillin-sulbactam, an $\mathrm{RCT}^{22}$ found no significant difference in clinical response rate between

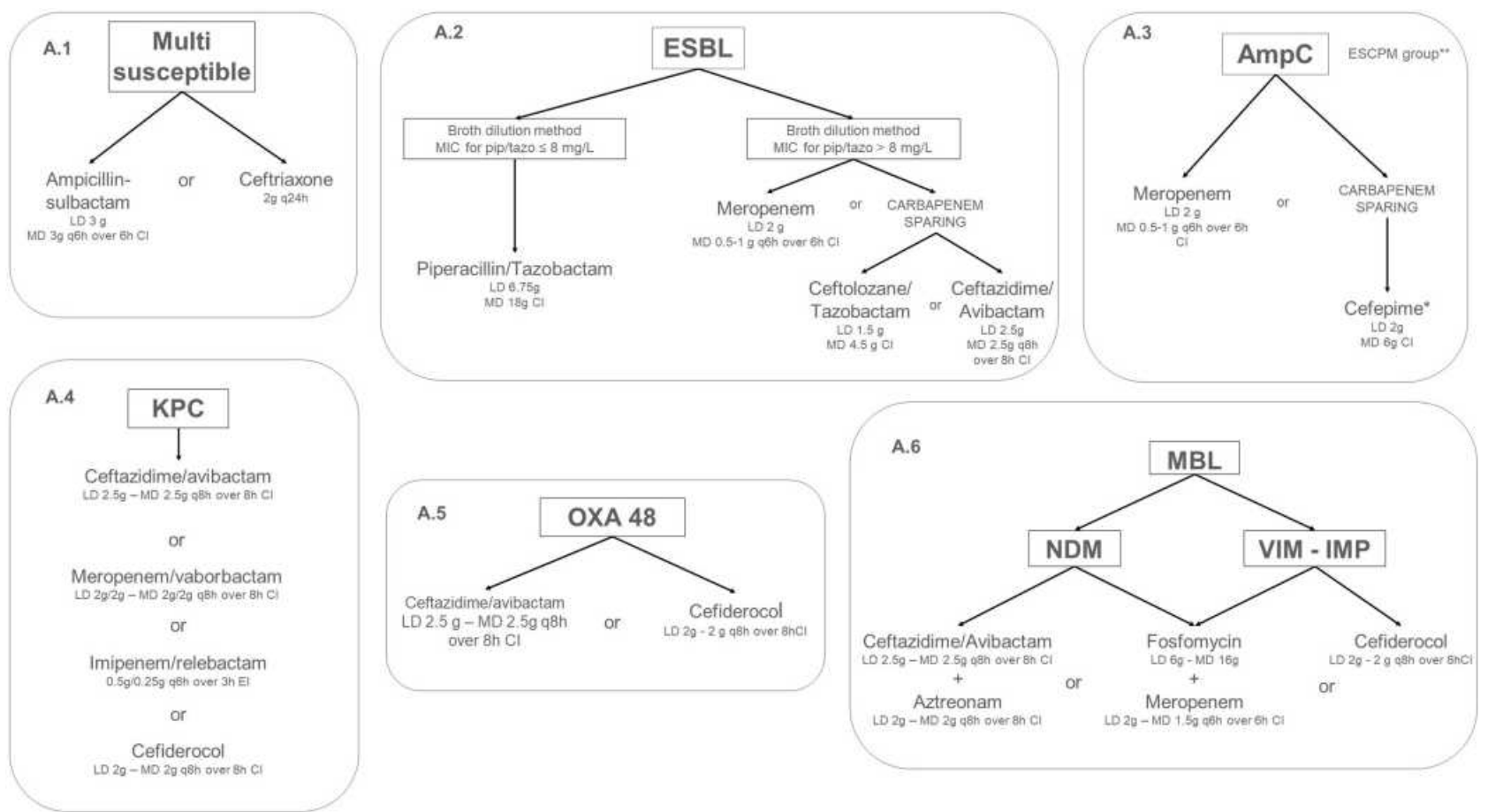

Figure 2 Algorithms for targeted treatment of BSI, cUTI and clAl, caused by Enterobacterales with different pattern of susceptibility in the ICU setting. *if MIC for cefepime $\leq \mathrm{Ig} / \mathrm{L}$. ** ESCPM group includes: Enterobacter (E. cloacae complex, E. aerogenes), Serratia marcescens, Citrobacter freundii, Providencia stuartii, and Morganella morganii. Abbreviations: $\mathrm{Cl}$, continuous infusion; $\mathrm{MIC}$, minimum inhibitory concentration. 


\begin{tabular}{|c|c|c|c|c|c|c|c|c|}
\hline 苞 & & 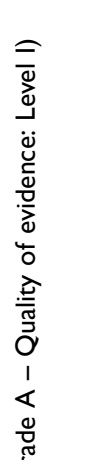 & 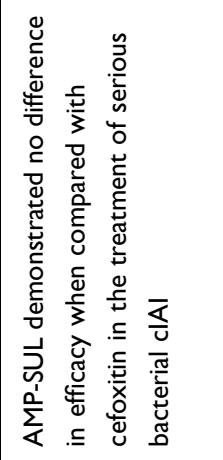 & 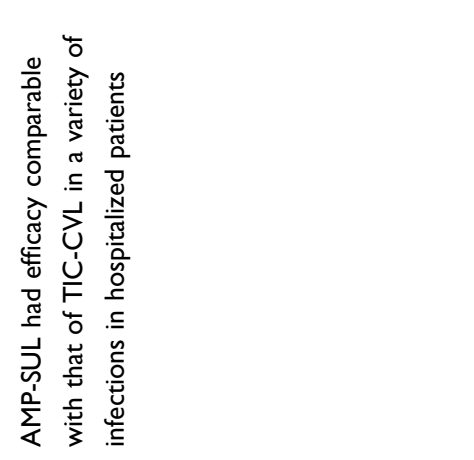 & & \multirow[t]{10}{*}{ 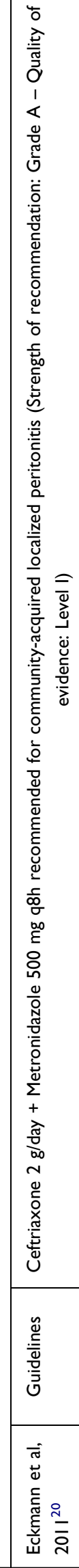 } & \multirow{10}{*}{ 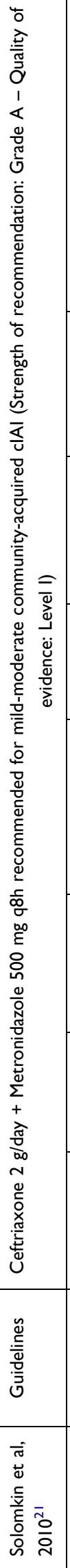 } & 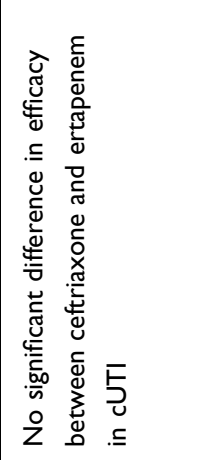 \\
\hline 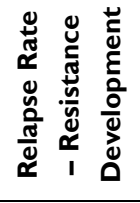 & & 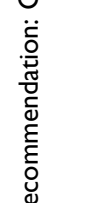 & 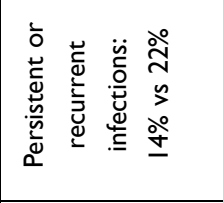 & 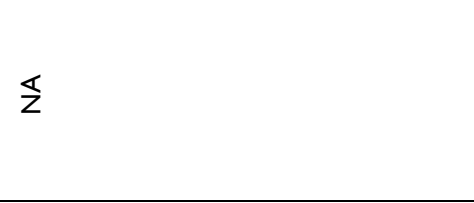 & & & & 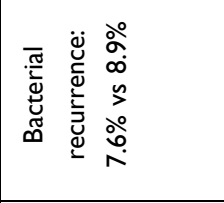 \\
\hline 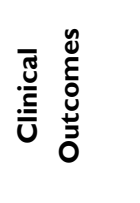 & & 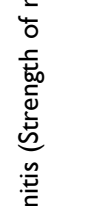 & 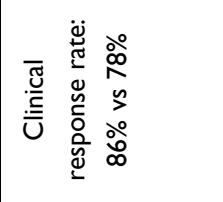 & 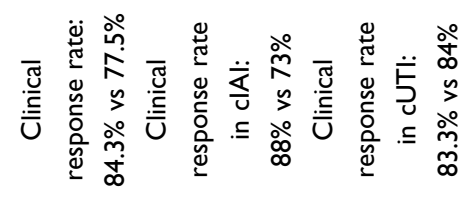 & & & & 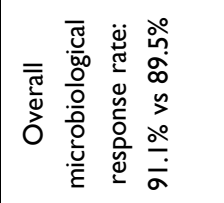 \\
\hline 离 & & 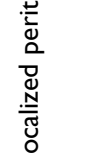 & 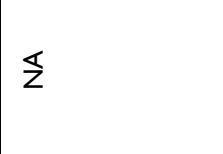 & 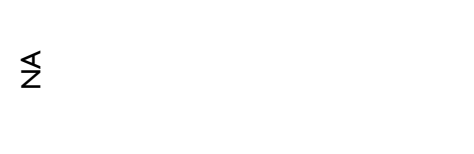 & & & & 竞 \\
\hline $\begin{array}{l}\underline{y} \\
\frac{ \pm}{0} \\
\underline{0}\end{array}$ & & 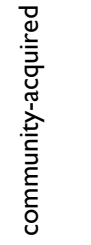 & 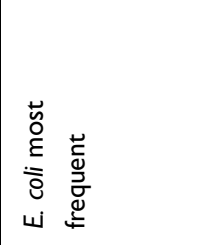 & 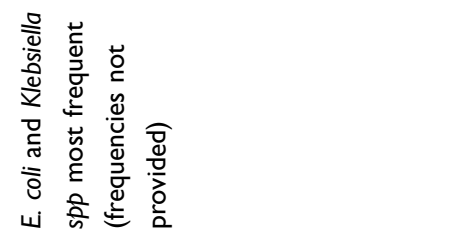 & & & & 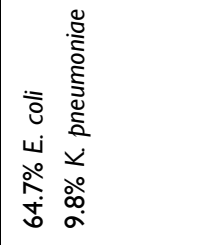 \\
\hline 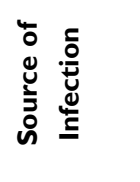 & & 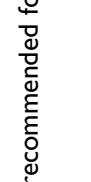 & $\overline{\mathrm{s}}$ & 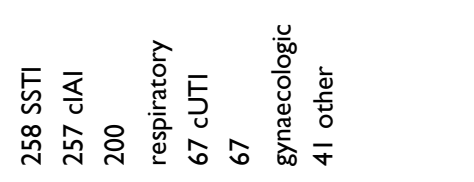 & & & & 5 \\
\hline 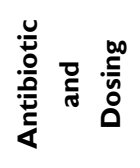 & & 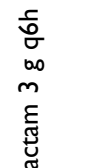 & 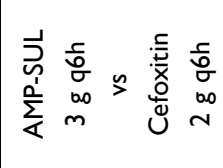 & 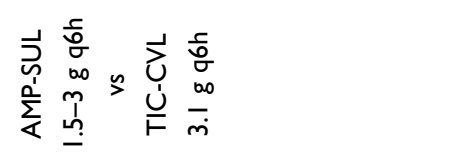 & & & & 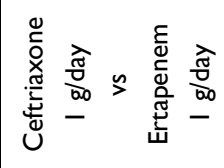 \\
\hline 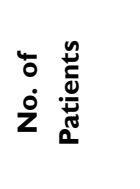 & & 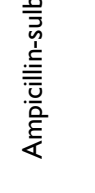 & 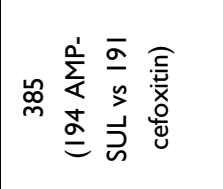 & 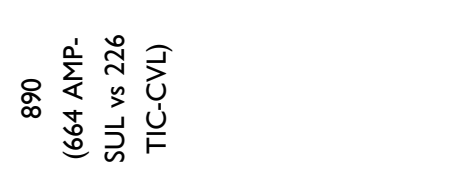 & & & & 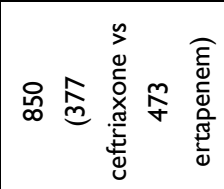 \\
\hline 㤹点 & & 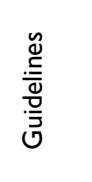 & 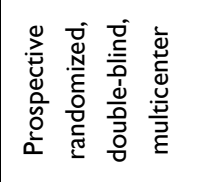 & 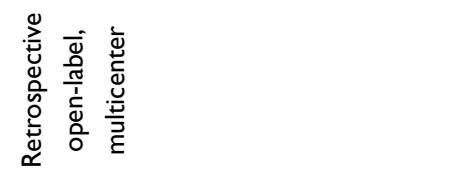 & & & & 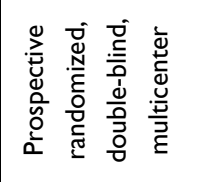 \\
\hline 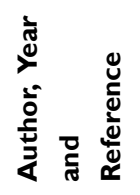 & 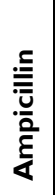 & 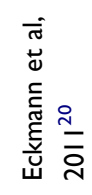 & 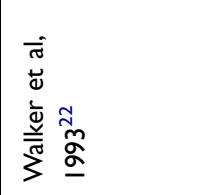 & 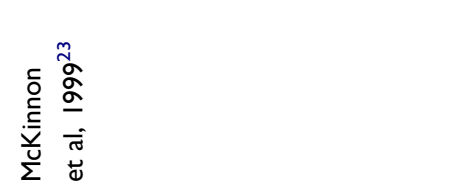 & 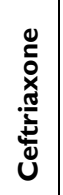 & & & 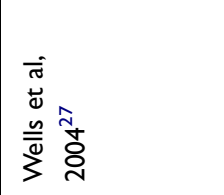 \\
\hline
\end{tabular}




\begin{tabular}{|c|c|c|c|}
\hline 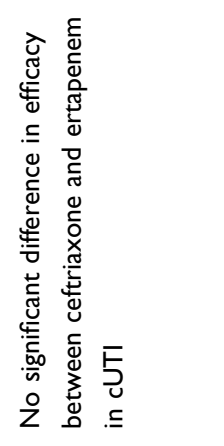 & 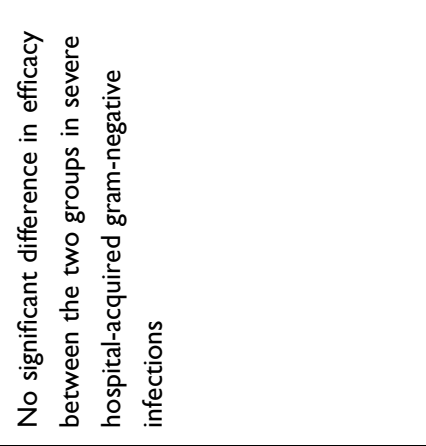 & 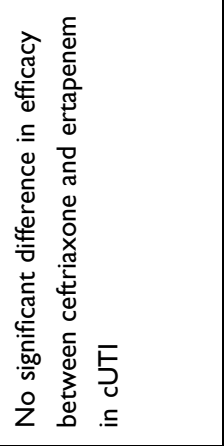 & 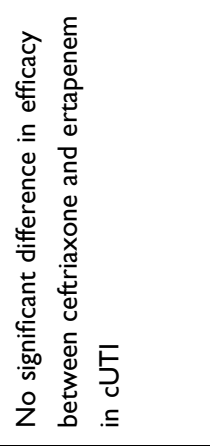 \\
\hline 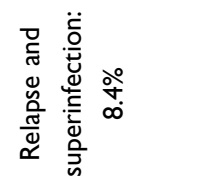 & 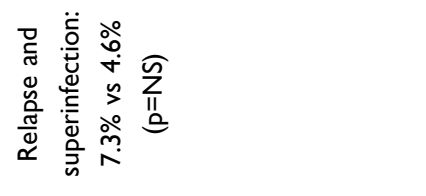 & $\S$ & 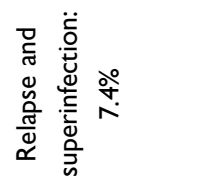 \\
\hline 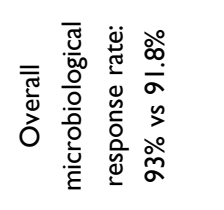 & 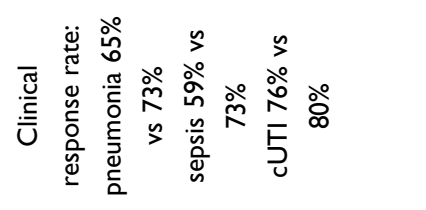 & 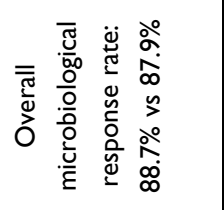 & 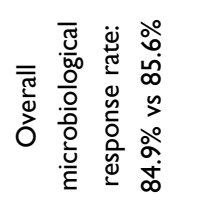 \\
\hline 怘 & 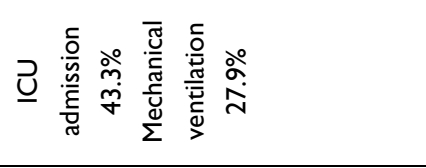 & 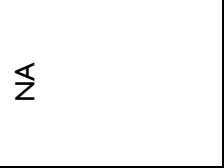 & 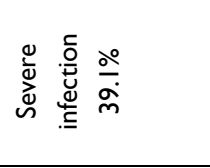 \\
\hline 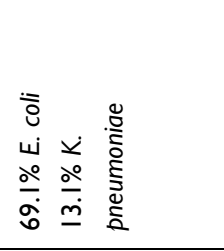 & 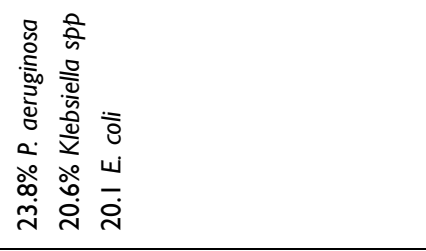 & 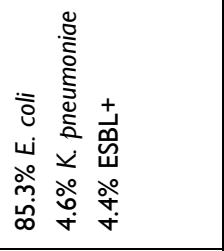 & 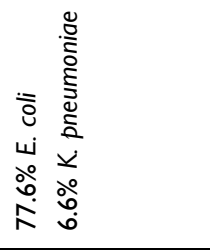 \\
\hline 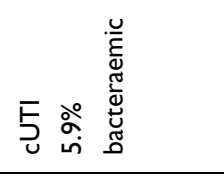 & 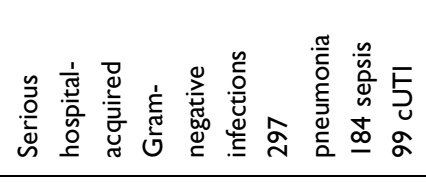 & F & క \\
\hline 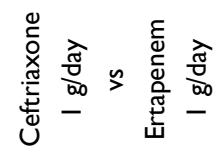 & 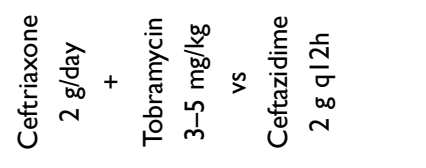 & 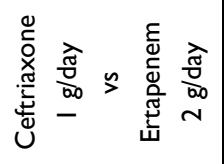 & 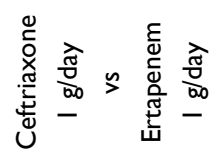 \\
\hline 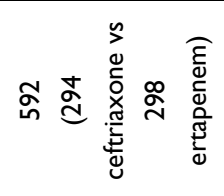 & 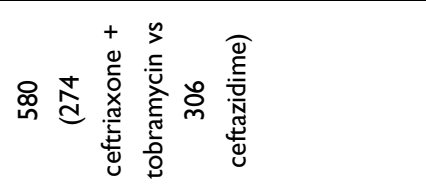 & 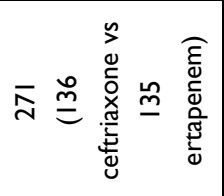 & 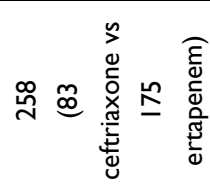 \\
\hline 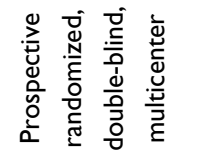 & 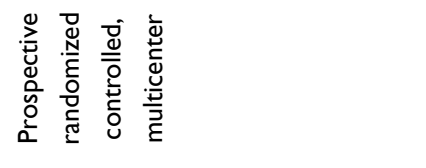 & 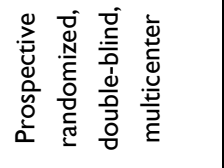 & 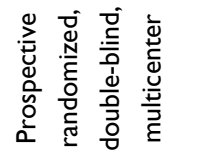 \\
\hline 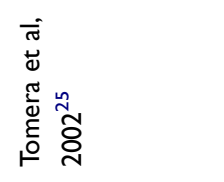 & 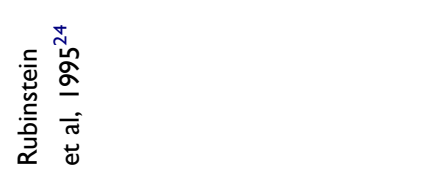 & 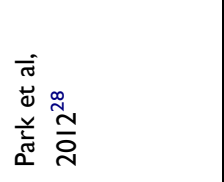 & 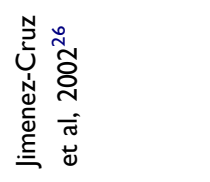 \\
\hline
\end{tabular}


ampicillin-sulbactam and cefoxitin (86\% vs 78\%) in patients affected by cIAIs mainly due to Escherichia coli, although features concerning infection severity were not provided. A retrospective observational study found comparable clinical response rate between ampicillin-sulbactam and ticarcillin-clavulanate $(84.3 \%$ vs $77.5 \%)$ among hospitalized patients with infections including cIAIs and cUTIs. ${ }^{23}$ Data on ICU admission and severity of infection were unavailable.

In regard to ceftriaxone, an RCT comparing ceftriaxone plus tobramycin versus ceftazidime in the treatment of severe hospital-acquired Gram-negative infections (including BSIs and cUTIs) showed no significant difference in clinical response rate among patients who required ICU admission in $43.3 \%$ of cases. ${ }^{24}$ Several RCTs ${ }^{25-28}$ showed no significant difference in terms of microbiological response rate between ceftriaxone and ertapenem for the management of cUTIs caused mainly by $E$. coli and $K$. pneumoniae. Almost $40 \%$ of patients had severe infection and relapse rate was $<10 \%$. cUTI were bacteraemic in 5.9$33.6 \%$ of cases may provide, and this may support the efficacy of ceftriaxone in BSIs as well.

\section{Extended-Spectrum Beta-Lactamase (ESBL)-Producing Enterobacterales}

Recommentations are depicted in Figure 2, panel A.2. Piperacillin-tazobactam (18g CI after $6.75 \mathrm{~g}$ LD) is recommended for the management of infections caused by ESBL-producing Enterobacterales with a piperacillintazobactam $\mathrm{MIC} \leq 8 \mathrm{mg} / \mathrm{L}$ (according to the EUCAST breakpoint) tested by broth microdilution (the reference method for piperacillin-tazobactam susceptibility testing). Conversely, meropenem (0.5-1g q6h over 6h CI after $2 \mathrm{~g}$ LD) should be preferred whenever piperacillin/tazobactam $\mathrm{MIC}$ is $>8 \mathrm{mg} / \mathrm{L}$. Ceftolozane-tazobactam $(1.5 \mathrm{~g} \mathrm{LD}$ followed by $4.5 \mathrm{~g} \mathrm{CI}$ ) and ceftazidime-avibactam $(2.5 \mathrm{~g} \mathrm{LD}$ followed by $2.5 \mathrm{~g}$ q $8 \mathrm{~h}$ over $8 \mathrm{~h} \mathrm{CI}$ ) may represent alternative options when focusing at a "carbapenem-sparing" approach. $^{29}$ In settings with high prevalence of carbapenem-resistant Enterobacterales (CRE), ceftazidime-avibactam should be reserved for CRE treatment to avoid epidemiological shift of carbapenemase producers to metallo-beta-lactamases (MBLs). ${ }^{29,30}$ Evidences supporting these choices are summarized in Table 2. Several studies compared piperacillin-tazobactam with carbapenems in the treatment of BSIs, cUTIs, and cIAIs caused by ESBL-producing Enterobacterales. Overall, an inconsistence emerged from the available evidence. The MERINO trial ${ }^{31}$ was the first large RCT that assessed the efficacy of a carbapenem-sparing strategy by comparing piperacillin-tazobactam vs meropenem in the treatment of ceftriaxone-resistant Escherichia coli or Klebsiella pneumoniae BSIs. Non-inferiority was not achieved in the piperacillin-tazobactam arm, as an overall 30-day allcause mortality rate 3 -fold higher than in the meropenem arm was observed $(12.3 \%$ vs $3.7 \%, \mathrm{p}=0.90)$. This arose concerns regarding the use of piperacillin-tazobactam as empirical or definitive therapy of ESBL-producing Enterobacterales BSIs. ${ }^{17}$ Indeed, it should be mentioned that a number of issues affected trial conclusions. ${ }^{17,32}$ The low mortality rate in the meropenem group was an unexpected finding; the primary source of BSI was imbalanced between arms (higher UTIs rate in the meropenem group); the number of neutropenic and immunocompromised patients was higher in the piperacillin-tazobactam group; sample size calculation was suboptimal; there was a high prevalence of $b l a_{\text {OXA-1 }}$ genes (67\%) strictly associated with high MICs for piperacillin-tazobactam (8-16 mg/L); ${ }^{33}$ pharmacokinetic/pharmacodynamic (PK/ PD) properties of piperacillin-tazobactam were not maximized (administration by intermittent rather than by prolonged infusion). Conversely, a large body of evidence coming from well-design observational studies and systematic review ${ }^{34-46}$ showed no significant difference in efficacy and mortality rate between piperacillin-tazobactam and carbapenems among patients with ESBL-producing primary or secondary BSI in settings with ICU admission rate up to $40 \%$. An RCT of piperacillin-tazobactam vs ertapenem found no significant difference in clinical cure and mortality rate among patients with bacteraemic cUTIs. ${ }^{47}$ Two studies showed a lower occurrence of MDR bacterial or fungal infections at 30-day from treatment with piperacillin-tazobactam compared to carbapenems $(7.4 \%$ vs $24.6 \% ; \mathrm{p}=0.01),{ }^{39}$ and a trend toward lower CRE isolation rate $(2 \%$ vs $8 \% ; \mathrm{p}=0.09) .{ }^{46}$ Conversely, two other observational studies showed significantly lower mortality rate in patients treated with carbapenems compared to piperacillin-tazobactam. ${ }^{48,49}$ However, in one of this ${ }^{48}$ some limits must be recognized, as $61 \%$ of patients received lower-than-desirable dosage of piperacillin-tazobactam $(3.375 \mathrm{~g} \mathrm{q} 6 \mathrm{~h})$ by intermittent infusion, and $60 \%$ of Enterobacterales isolates had piperacillin-tazobactam MIC $\geq 8 \mathrm{mg} / \mathrm{L}$. Overall, the available literature may provide support for considering piperacillin-tazobactam a valuable carbapenem-sparing agent in the 


\begin{tabular}{|c|c|c|c|}
\hline 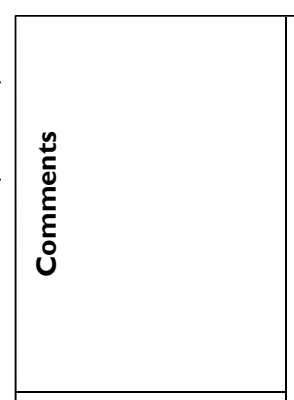 & 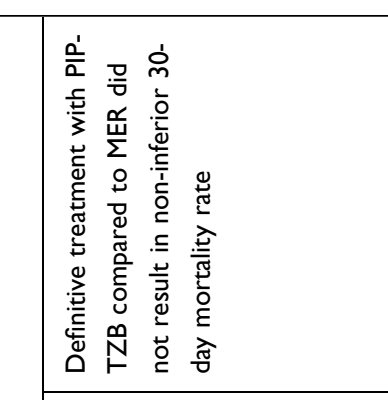 & 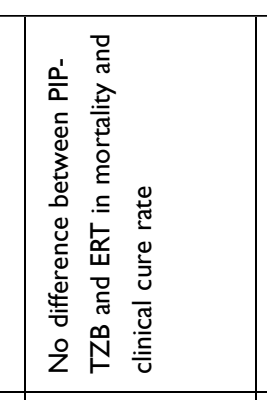 & 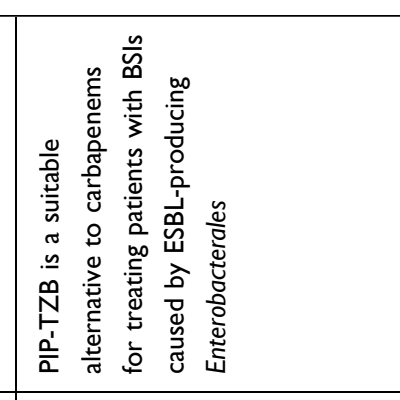 \\
\hline 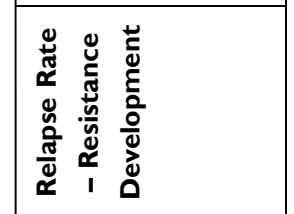 & $\frac{\pi}{z}$ & 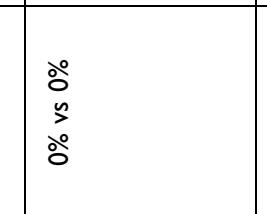 & $\frac{\pi}{z}$ \\
\hline 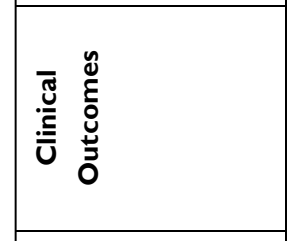 & 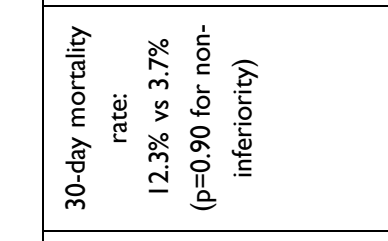 & 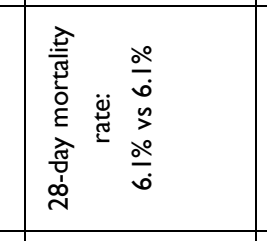 & 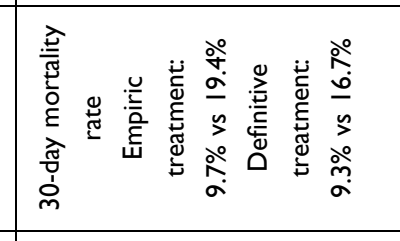 \\
\hline 言 & 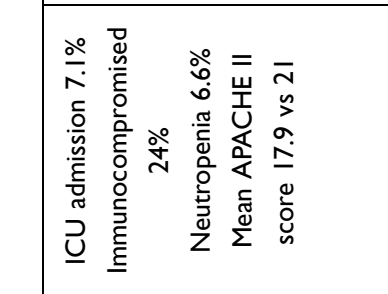 & 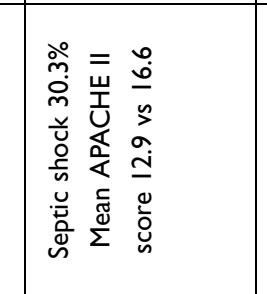 & 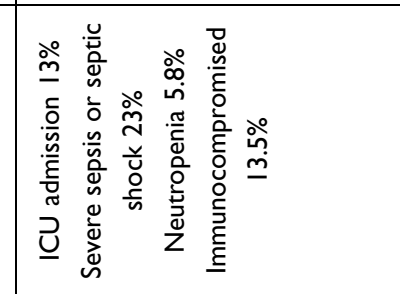 \\
\hline 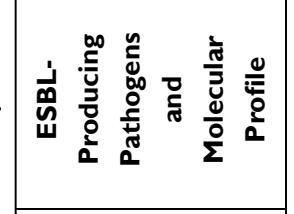 & 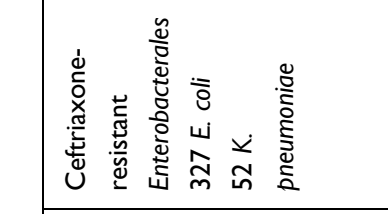 & 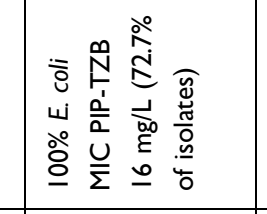 & 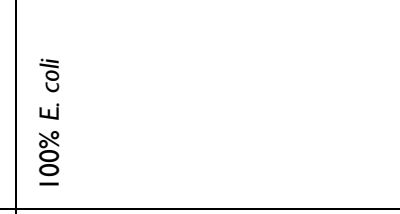 \\
\hline 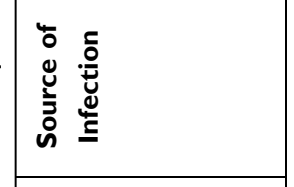 & 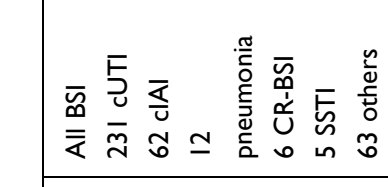 & 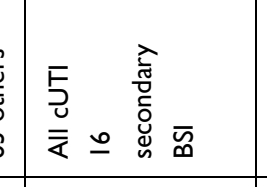 & 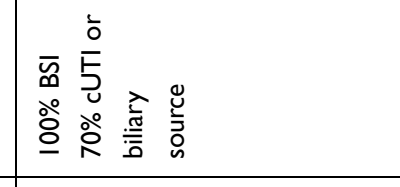 \\
\hline 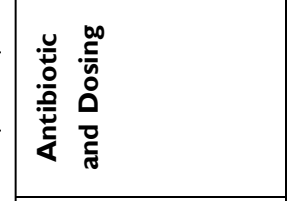 & 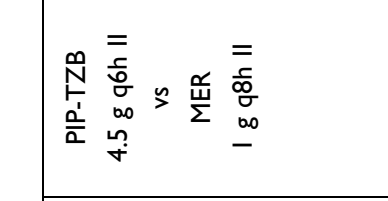 & 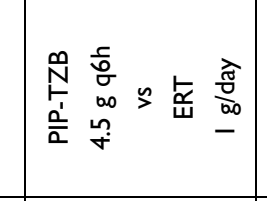 & 童点 \\
\hline 资 & 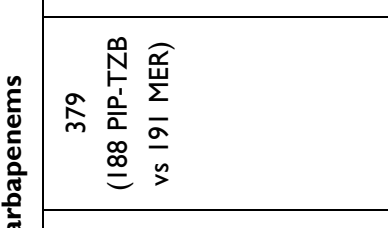 & 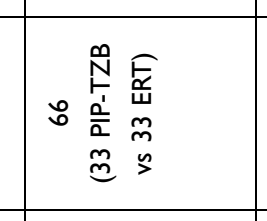 & 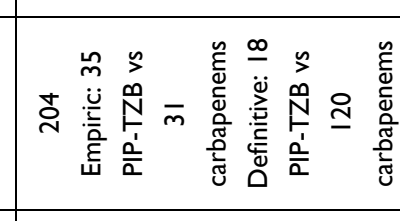 \\
\hline 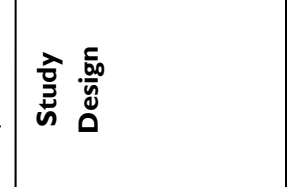 & 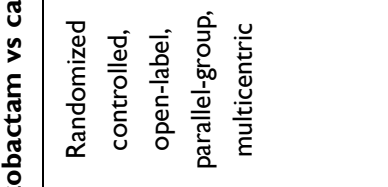 & 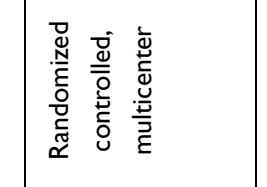 & 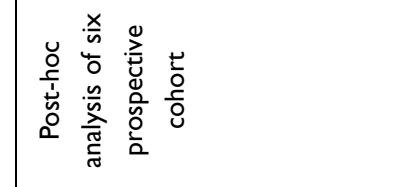 \\
\hline 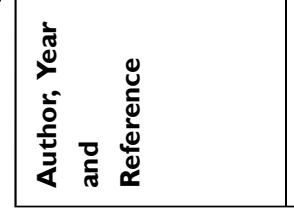 & 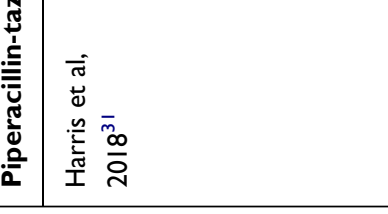 & 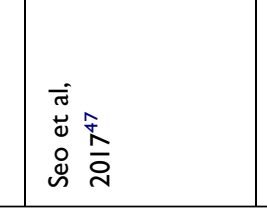 & 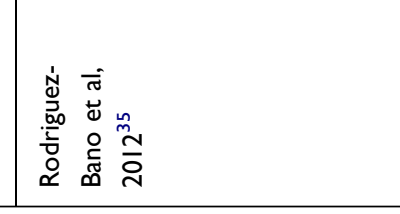 \\
\hline
\end{tabular}




\begin{tabular}{|c|c|c|c|}
\hline 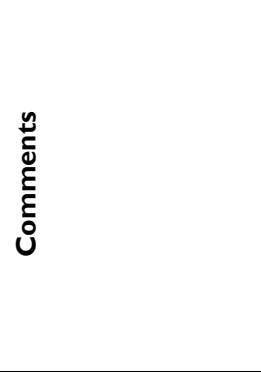 & 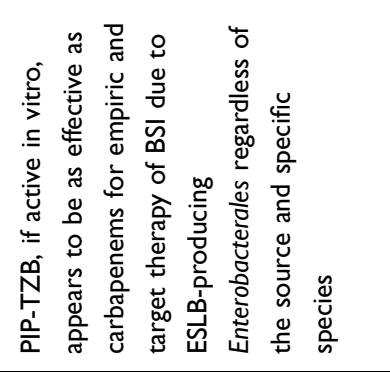 & 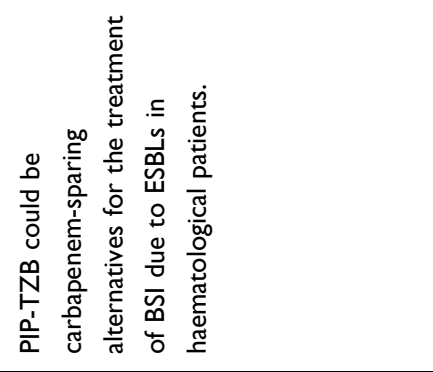 & 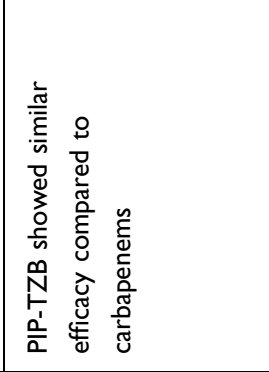 \\
\hline 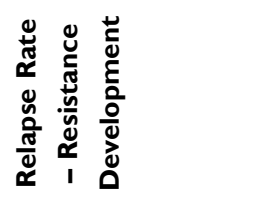 & $\mathbb{Z}$ & 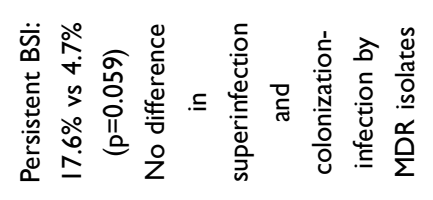 & $\mathbb{z}$ \\
\hline 氖 & 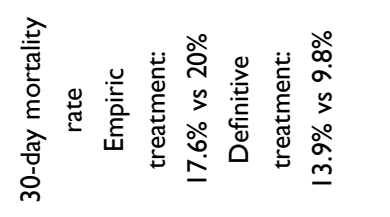 & 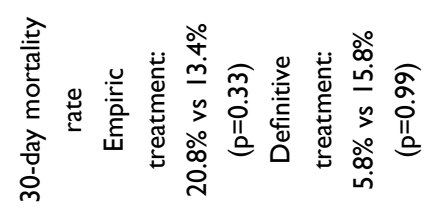 & 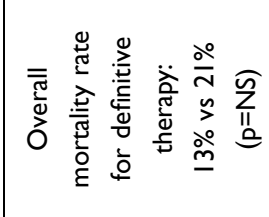 \\
\hline 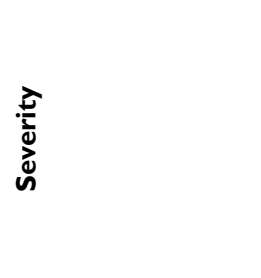 & 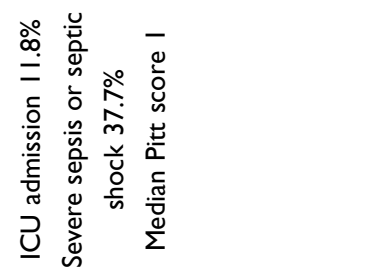 & 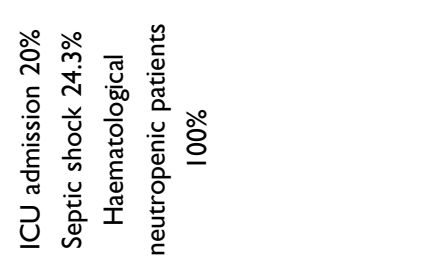 & 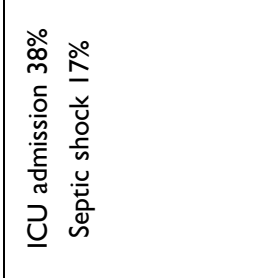 \\
\hline 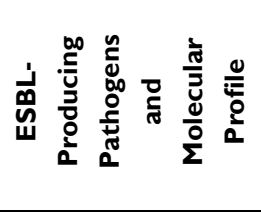 & 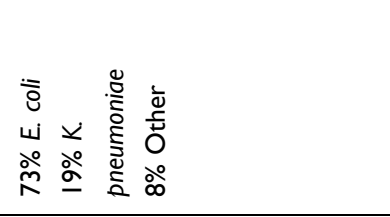 & 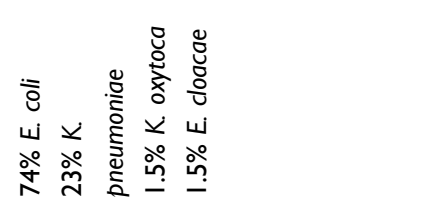 & 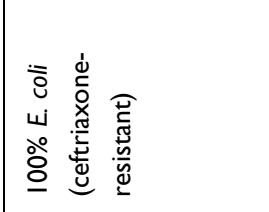 \\
\hline 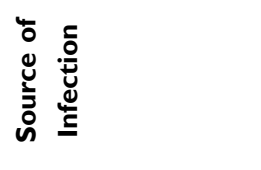 & 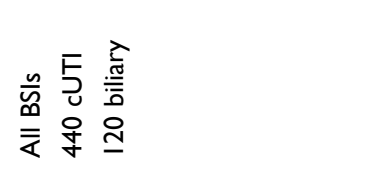 & 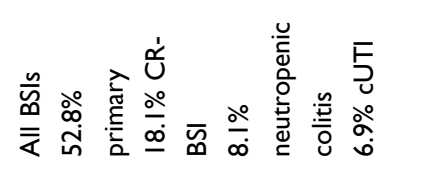 & 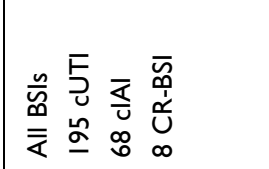 \\
\hline 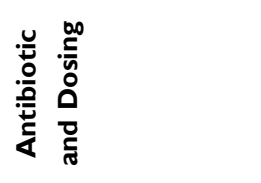 & 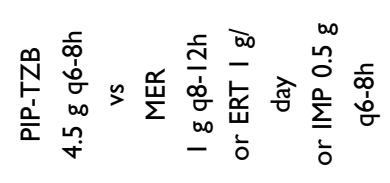 & 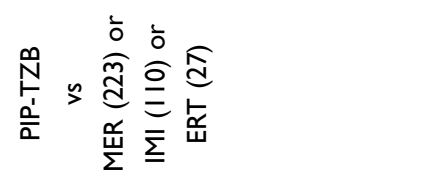 & 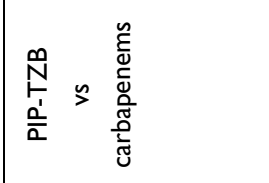 \\
\hline 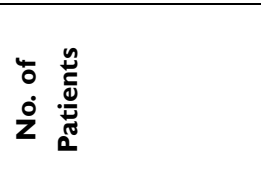 & 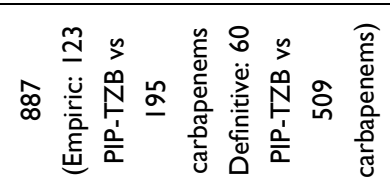 & 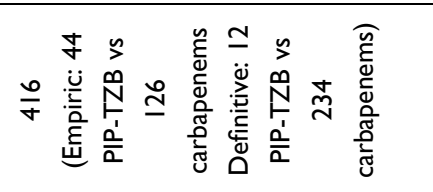 & 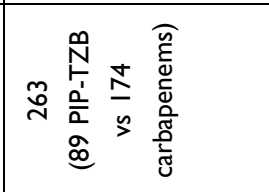 \\
\hline 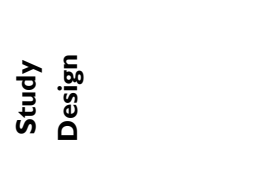 & 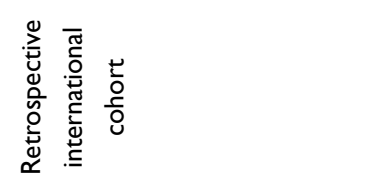 & 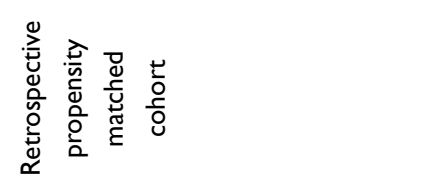 & 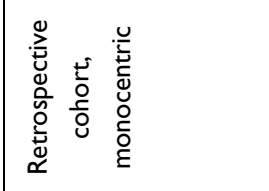 \\
\hline 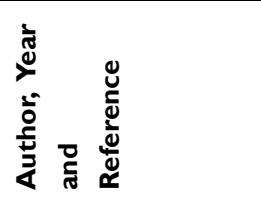 & 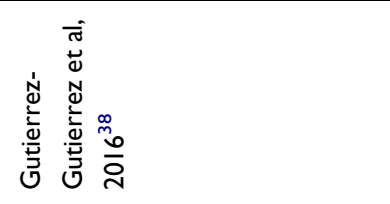 & 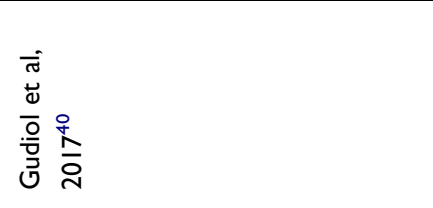 & 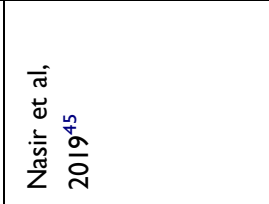 \\
\hline
\end{tabular}




\begin{tabular}{|c|c|c|}
\hline 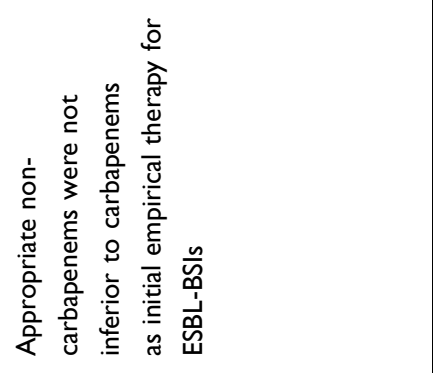 & 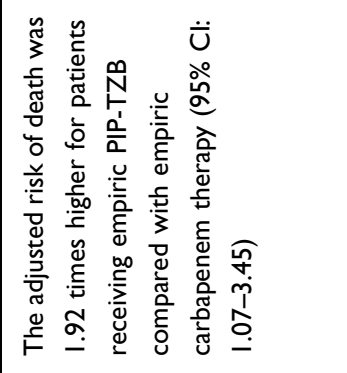 & 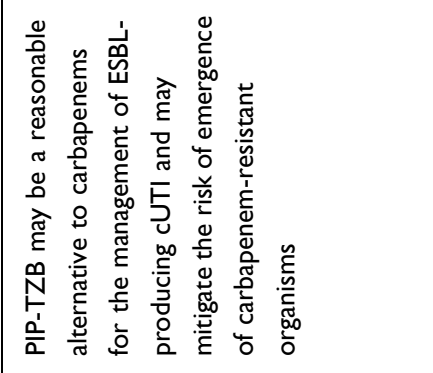 \\
\hline$\underline{z}$ & $\mathbb{z}$ & 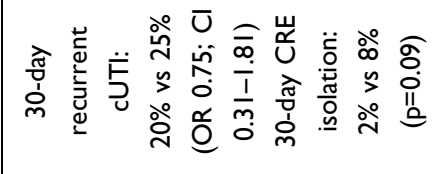 \\
\hline 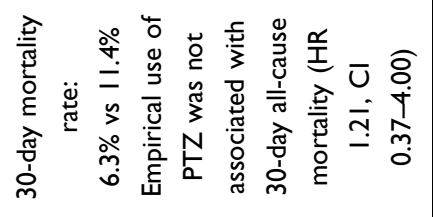 & 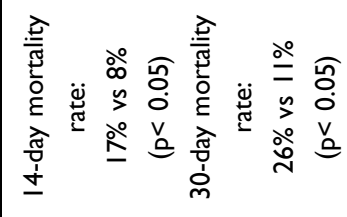 & 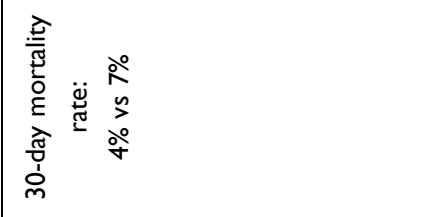 \\
\hline 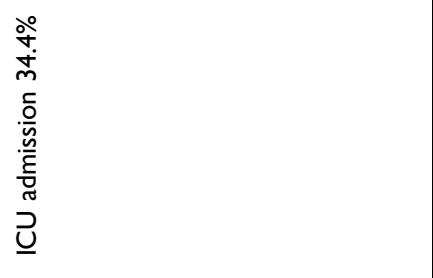 & 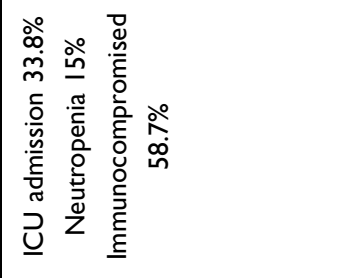 & 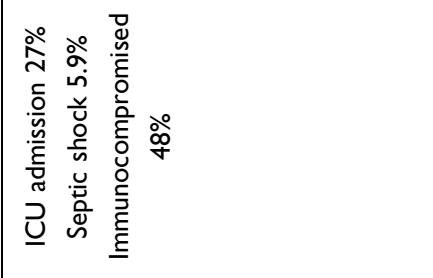 \\
\hline 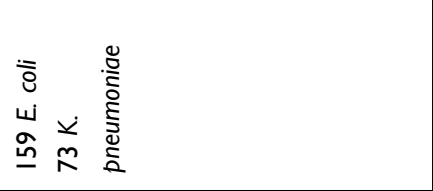 & 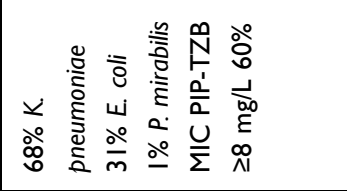 & 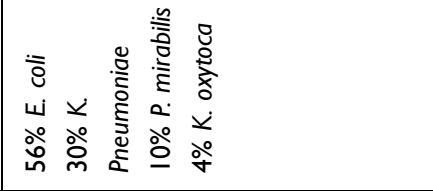 \\
\hline 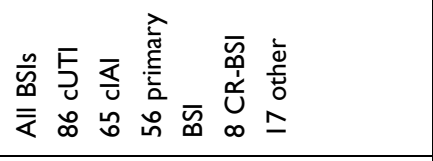 & 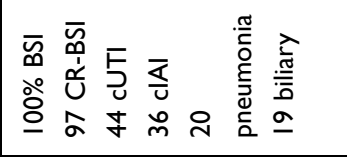 & 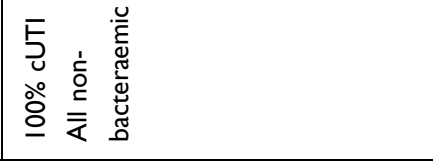 \\
\hline 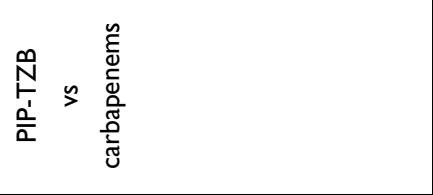 & 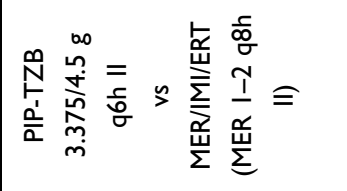 & 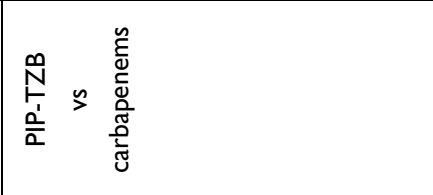 \\
\hline 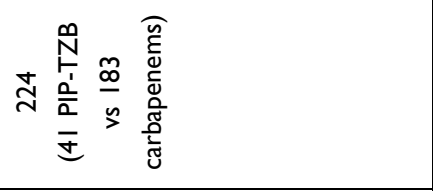 & 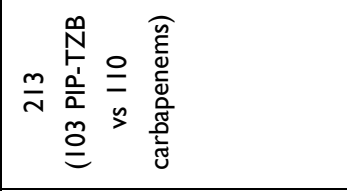 & 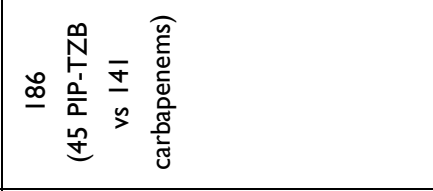 \\
\hline 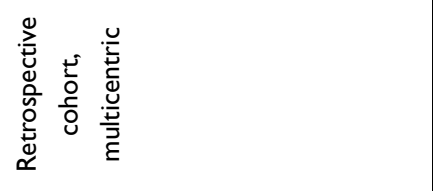 & 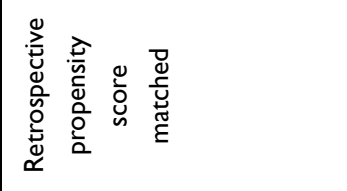 & 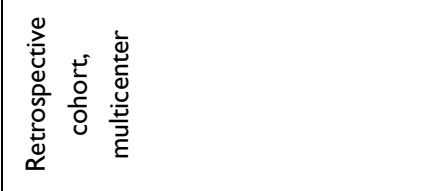 \\
\hline 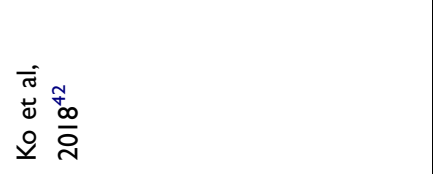 & 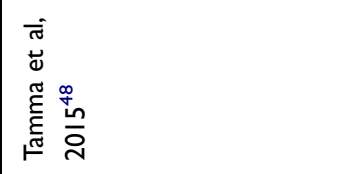 & 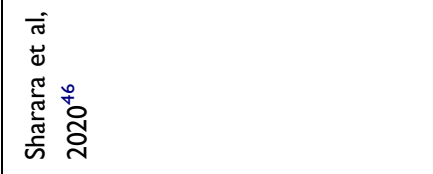 \\
\hline
\end{tabular}




\begin{tabular}{|c|c|c|c|c|}
\hline 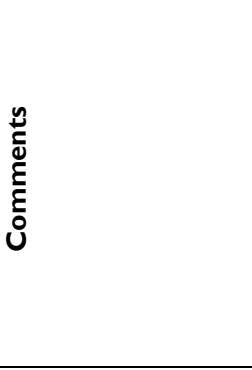 & 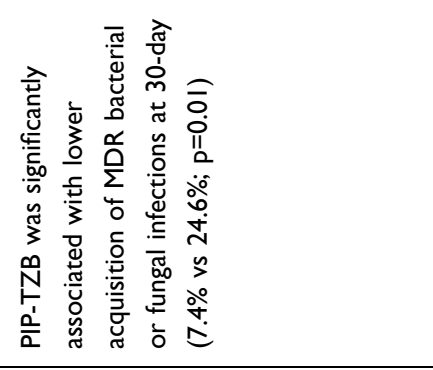 & 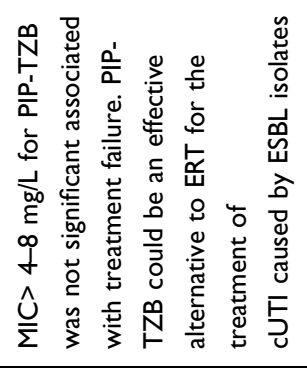 & 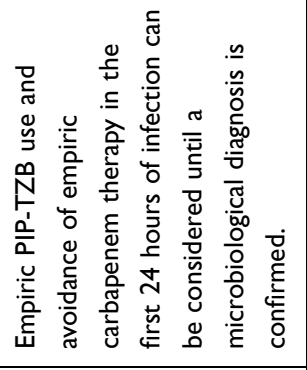 & 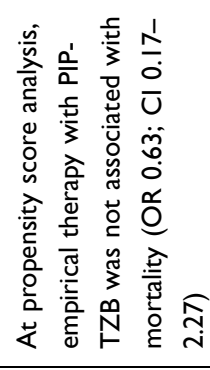 \\
\hline 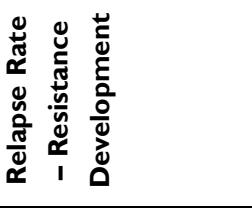 & 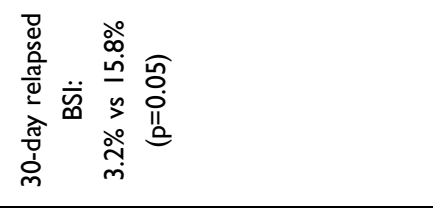 & $\S$ & 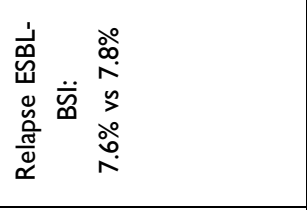 & $\S$ \\
\hline 胥 & 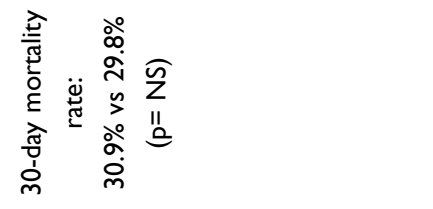 & 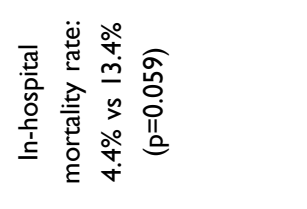 & 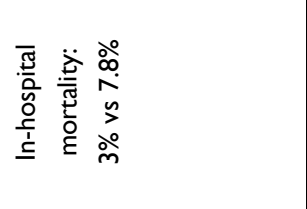 & 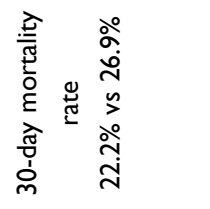 \\
\hline & 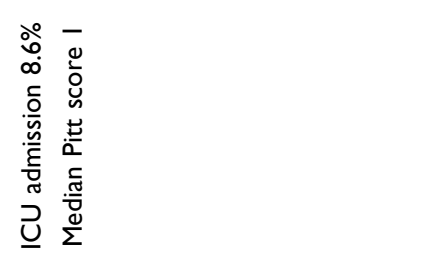 & 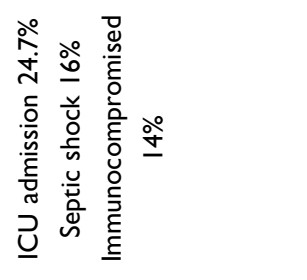 & 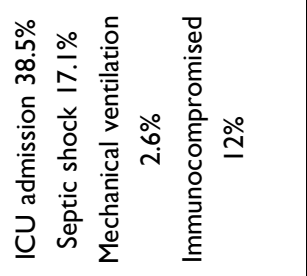 & 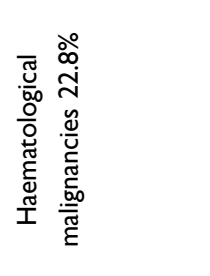 \\
\hline 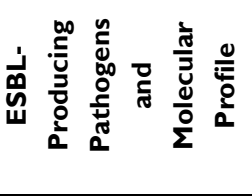 & 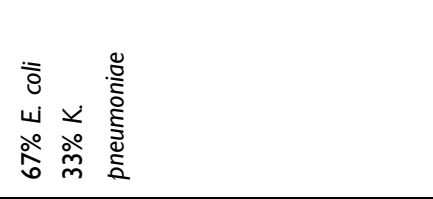 & $\begin{array}{l}\dot{\bar{c}} \\
\dot{j} \\
\stackrel{\circ}{\circ} \\
\underline{o}\end{array}$ & 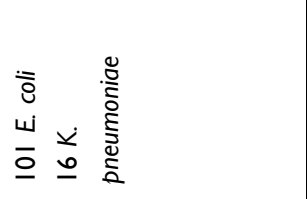 & 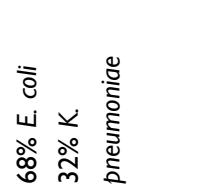 \\
\hline 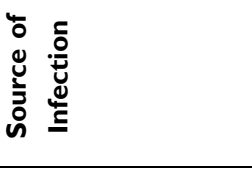 & 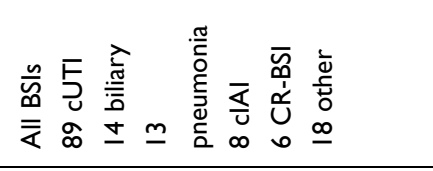 & 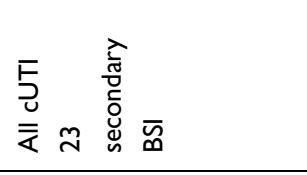 & 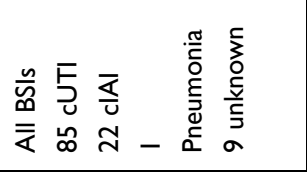 & 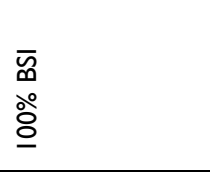 \\
\hline 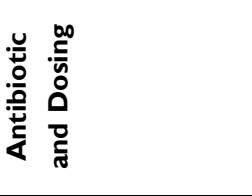 & 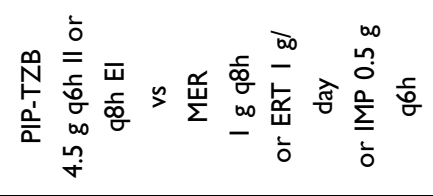 & 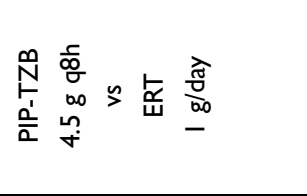 & 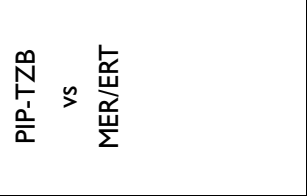 & 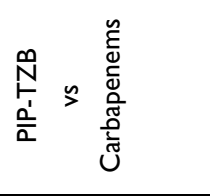 \\
\hline 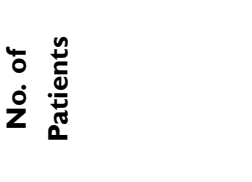 & 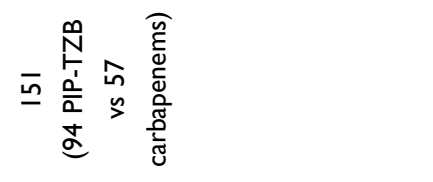 & 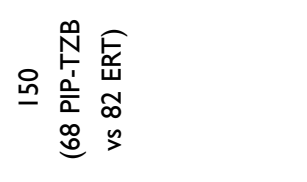 & 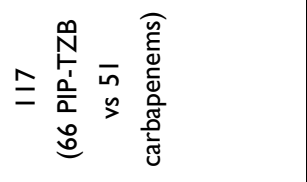 & 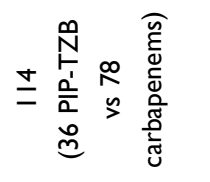 \\
\hline 昰高 & 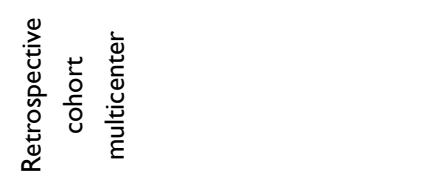 & 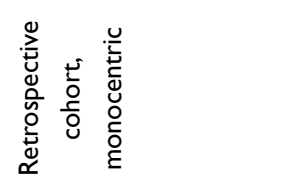 & 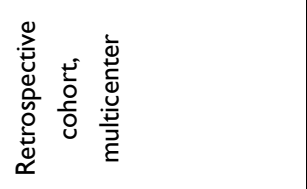 & 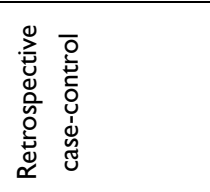 \\
\hline 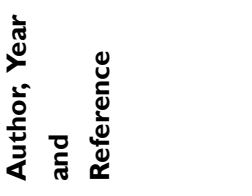 & 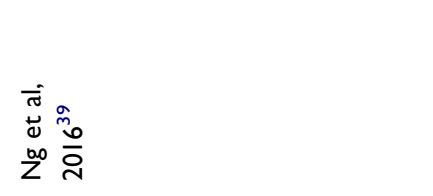 & 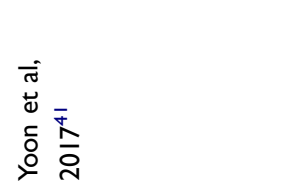 & 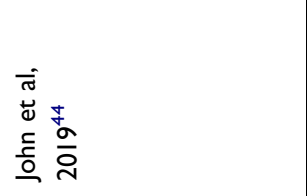 & 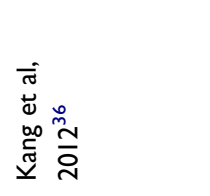 \\
\hline
\end{tabular}




\begin{tabular}{|c|c|c|}
\hline 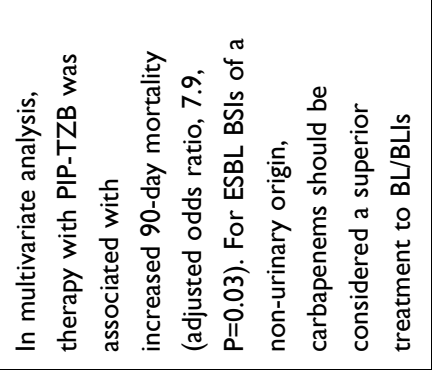 & 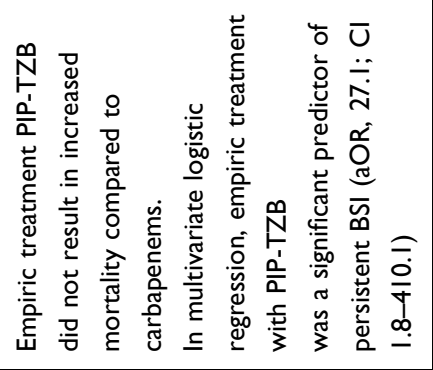 & 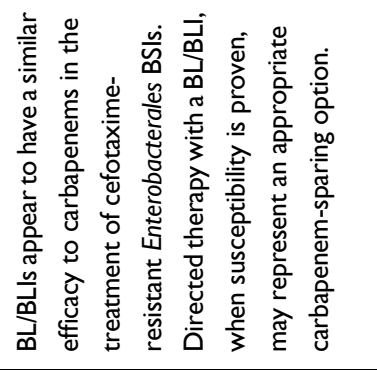 \\
\hline$\S$ & 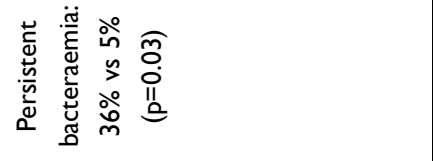 & $\begin{array}{l}\text { خे } \\
\text { 号 } \\
\text { ठे }\end{array}$ \\
\hline 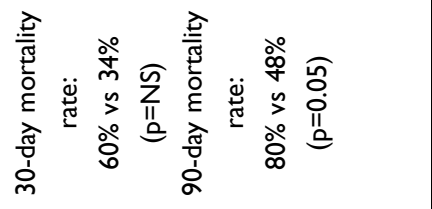 & 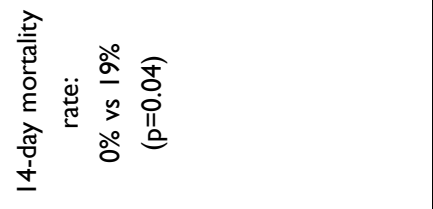 & 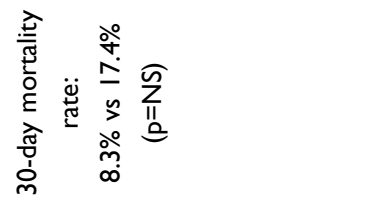 \\
\hline 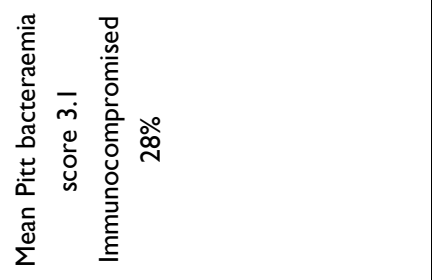 & 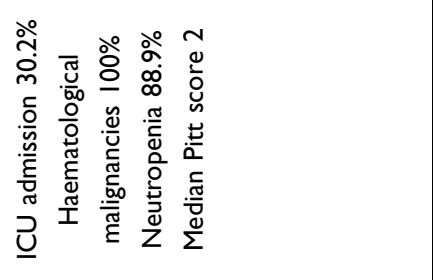 & 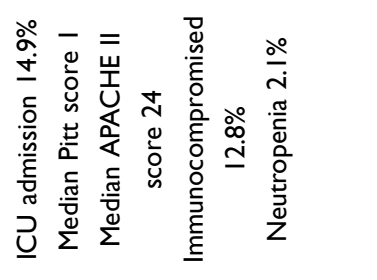 \\
\hline 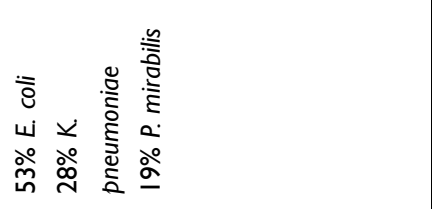 & 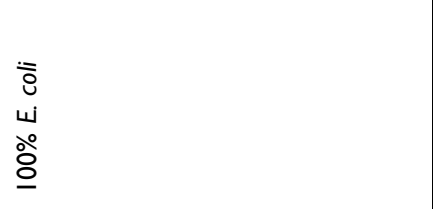 & 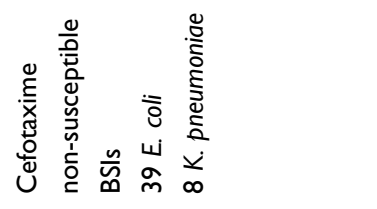 \\
\hline 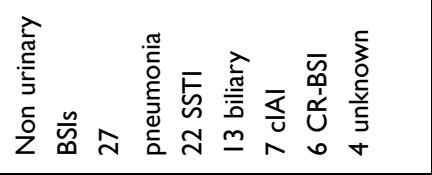 & 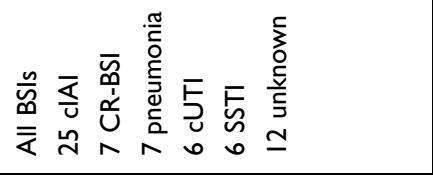 & 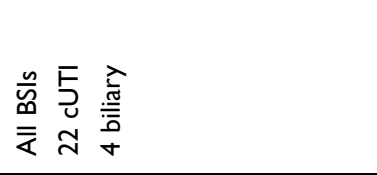 \\
\hline 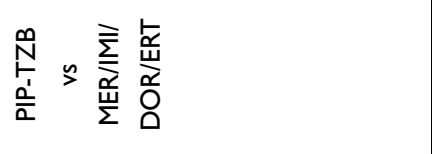 & 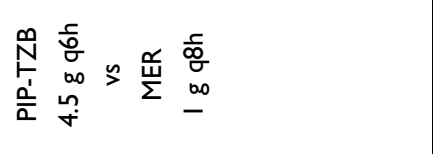 & 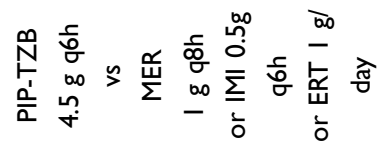 \\
\hline 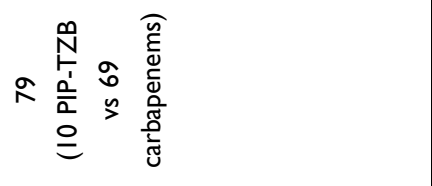 & 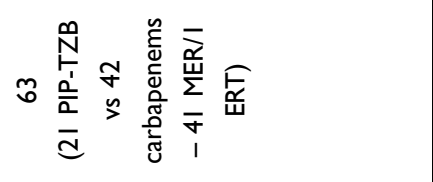 & 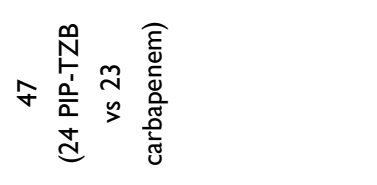 \\
\hline 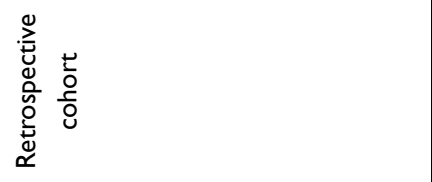 & 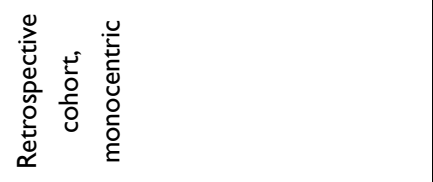 & 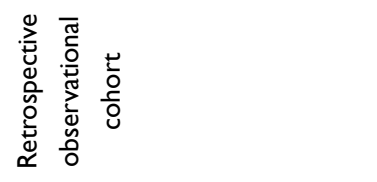 \\
\hline 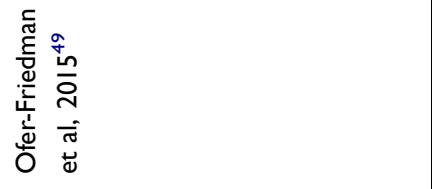 & 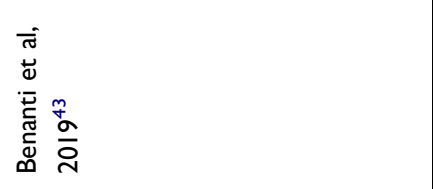 & 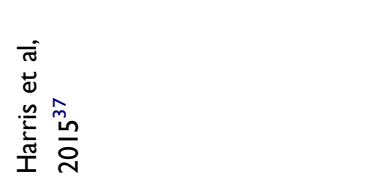 \\
\hline
\end{tabular}




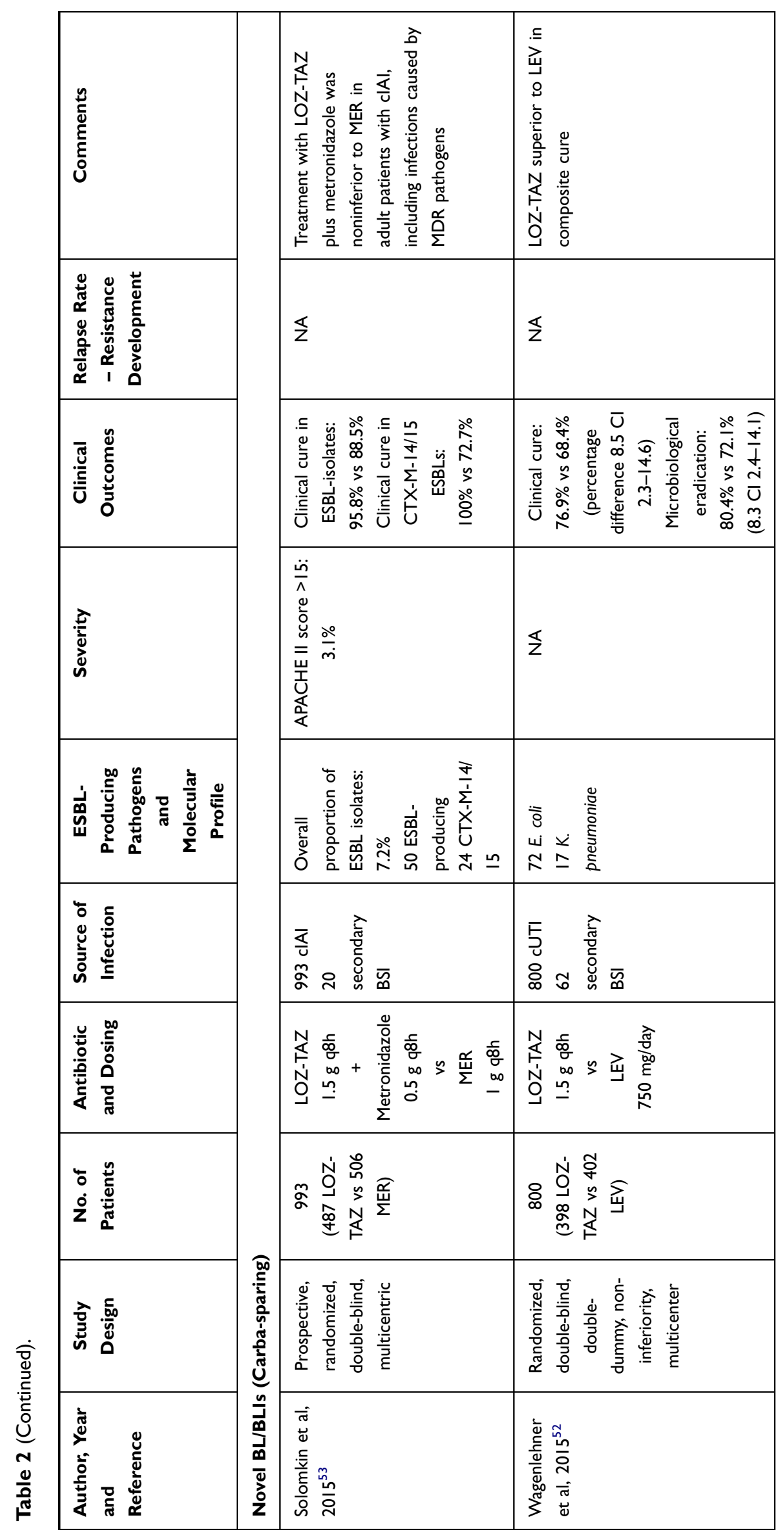




\begin{tabular}{|c|c|}
\hline 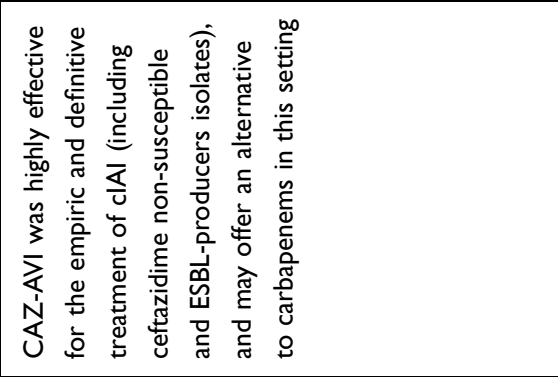 & 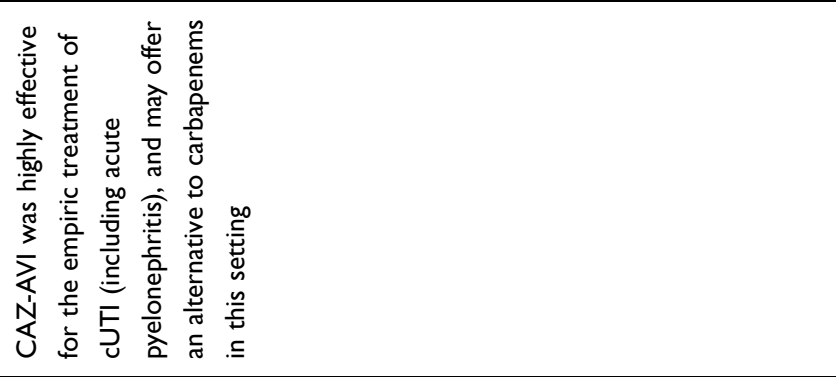 \\
\hline 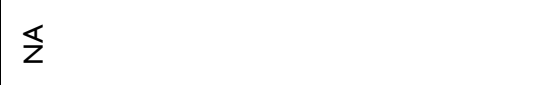 & $\mathbb{z}$ \\
\hline 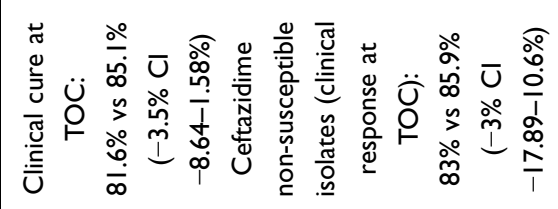 & 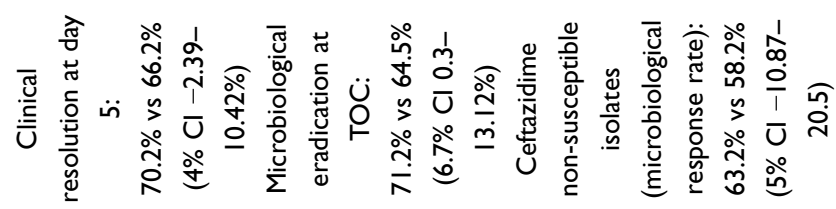 \\
\hline 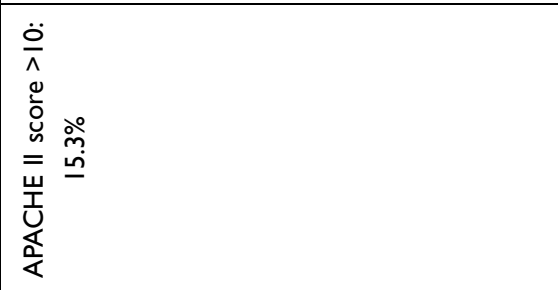 & 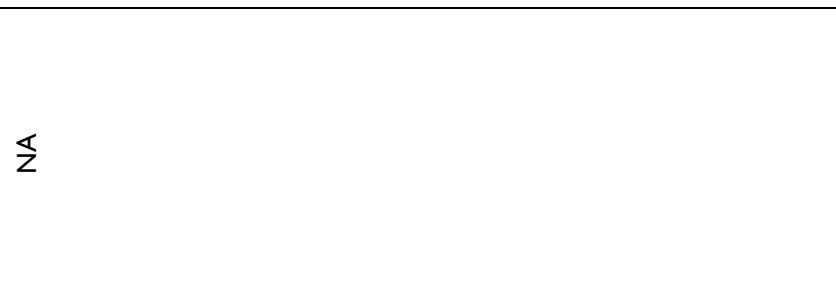 \\
\hline 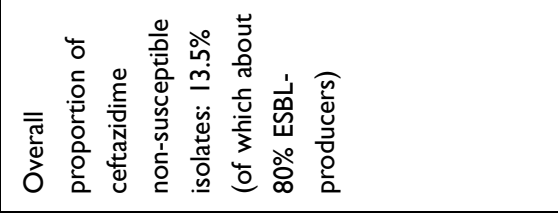 & 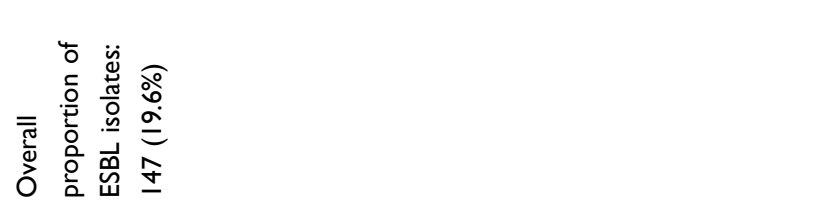 \\
\hline $\begin{array}{l}\bar{s} \\
\mathrm{u} \\
\mathrm{o} \\
\text { o }\end{array}$ & 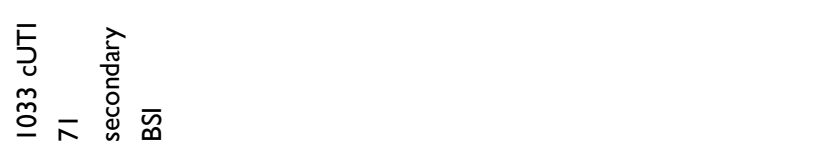 \\
\hline 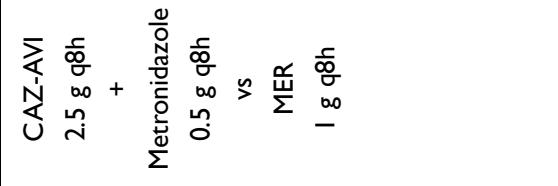 & 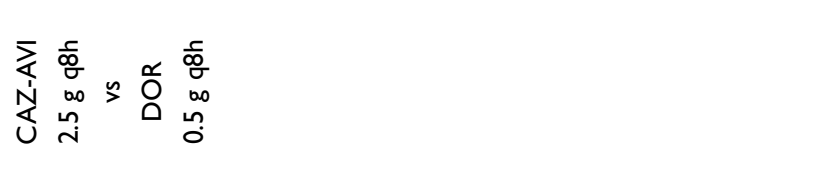 \\
\hline 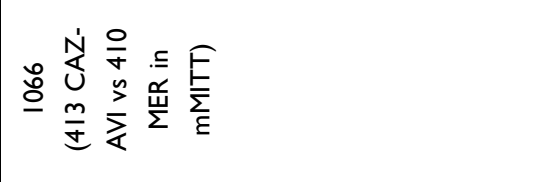 & 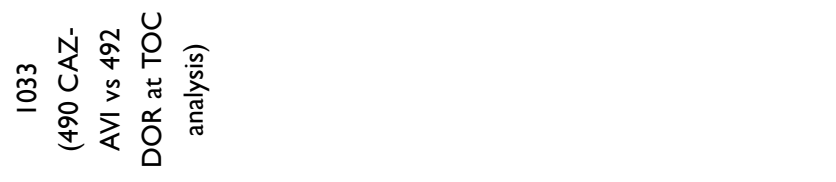 \\
\hline 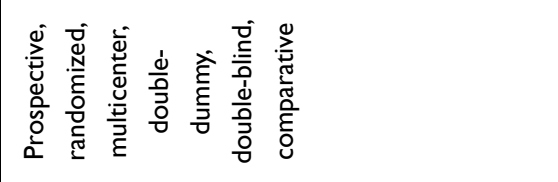 & 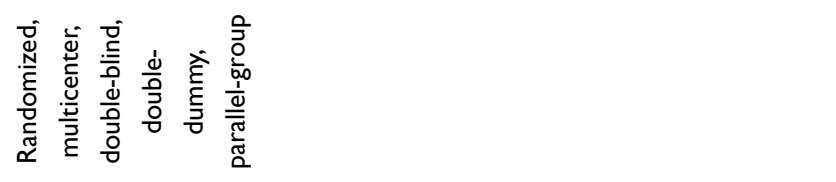 \\
\hline 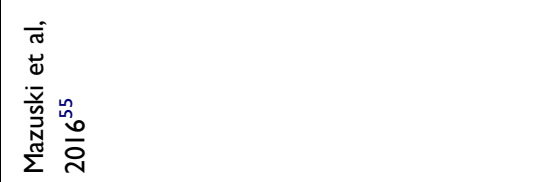 & 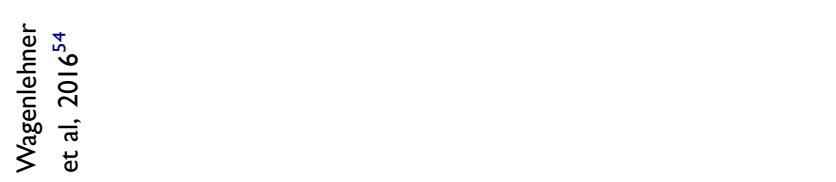 \\
\hline
\end{tabular}




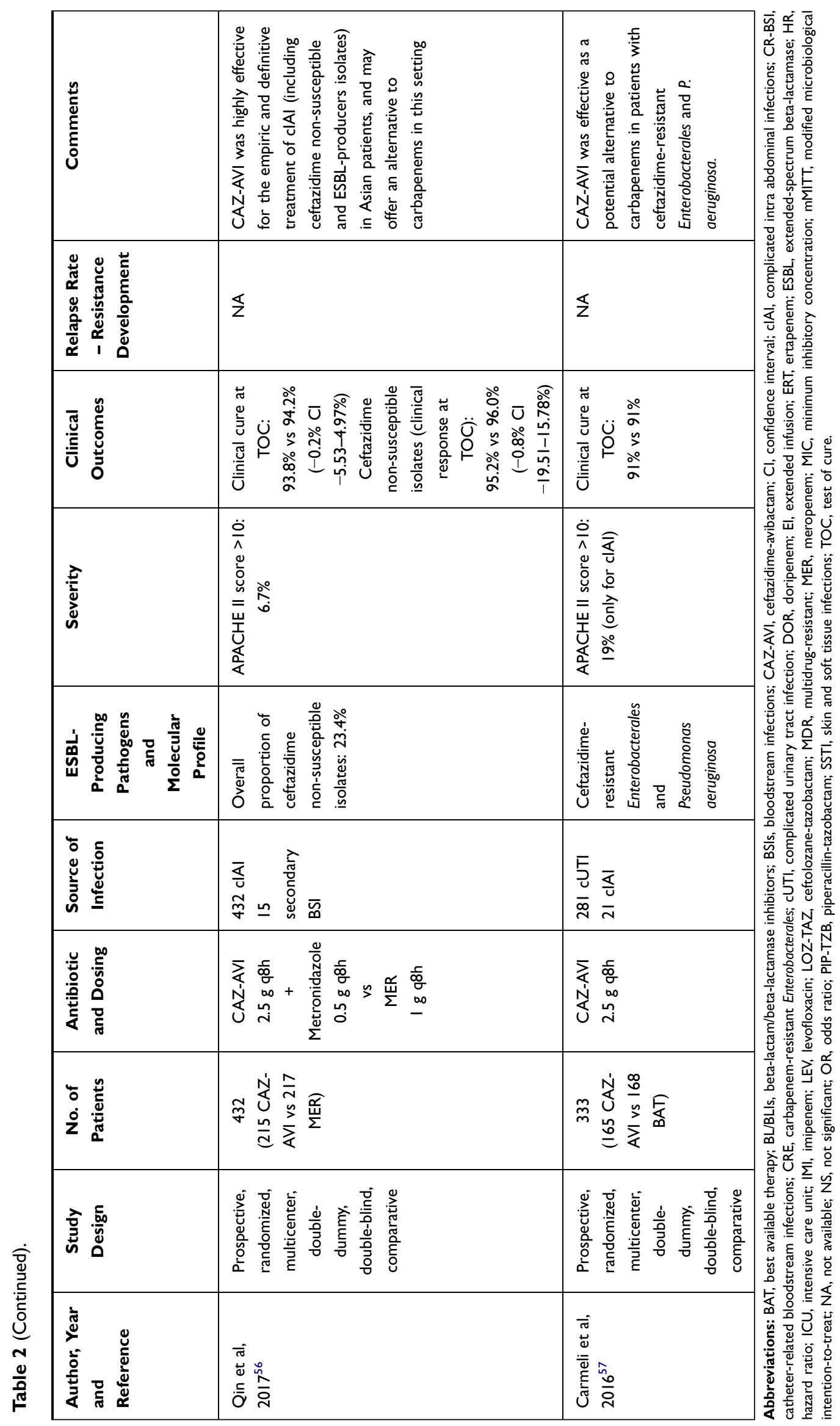


management of ESBL-related infections when dealing with fully susceptible pathogens (MIC $\leq 8 \mathrm{mg} / \mathrm{L}$ ). Notably, CI of high-dose piperacillin-tazobactam (18g) should be strongly recommended to achieve optimal PK/ PD target in critically ill patients affected by ESBL-producing Enterobacterales infections, especially when dealing with isolates exhibiting high MIC values. ${ }^{50,51}$ Evidence supporting ceftolozane-tazobactam and/or ceftazidime-avibactam as carbapenem-sparing options for the treatment of for BSIs, cUTIs, and cIAIs caused by ESBL-producing Enterobacterales came from the non-inferiority showed vs carbapenems in pivotal Phase III trials. ${ }^{52-57}$ Indeed, it should be recognized that the overall proportion of ESBL-producing isolates was $100 \%$ only in one study that enrolled exclusively patients with documented ceftazidime-resistant infections, ${ }^{57}$ whereas it was $<20 \%$ in all of the others.

\section{AmpC Beta-Lactamase-Producing Enterobacterales}

Recommendations are depicted in Figure 2, panel A.3. Meropenem (0.5-1g q6h over 6h CI after $2 \mathrm{~g}$ LD) is recommended as first-line treatment for BSIs, cUTIs, and cIAIs caused by AmpC-producing Enterobacterales. Cefepime (6g CI after 2g LD) may be a "carbapenemsparing" alternative option if the MIC is $\leq 1 \mathrm{mg} / \mathrm{L}$. AmpC beta-lactamases belong to the class $\mathrm{C}$ of the Ambler's classification, and genes encoding for them are usually located in the chromosome of bacteria belonging to the so-called ESCPM group (namely Enterobacter cloacae complex, Enterobacter aerogenes, Serratia marcescens, Citrobacter freundii, Providencia stuartii, and Morganella morganii), ${ }^{58}$ but can also be carried on transferable plasmids and found in isolates of other species (eg, E. coli, K. pneumoniae, P. mirabilis). AmpC may hydrolyse all the penicillins, the $1^{\text {st }}, 2^{\text {nd }}$ and $3^{\text {rd }}$ cephalosporins, and the monobactam aztreonam, but not the $4^{\text {th }}$ generation cephalosporins and the carbapenems. Furthermore, betalactamase inhibitors (tazobactam, sulbactam, clavulanate) exhibit no activity against AmpC-producing isolates. Evidences coming from comparative studies between carbapenems and cefepime in the treatment of BSIs, cUTIs, and cIAIs caused by AmpC-producing Enterobacterales is provided in Table 3. Overall, a large body of evidence obtained from well-design prospective and retrospective observational studies and from systematic reviews showed no significant difference in terms of clinical cure and mortality rate between cefepime and carbapenems in settings characterized by ICU admission up to $60 \%{ }^{59-66}$ However, it should not be overlooked that cefepime was less effective against strains with an MIC $\geq 2 \mathrm{mg} / \mathrm{L}$. One study showed significantly higher mortality rate in patients affected by cefepime-susceptible dose dependent (SDD) isolates (MIC 4-8 mg/L) who were treated with cefepime compared to those treated with carbapenems $(71.4 \%$ vs $18.2 \% ; \mathrm{p}=0.045)$, even if a full-dose cefepime $(6 \mathrm{~g} /$ day $)$ was administered only in $38.6 \%$ of cases. ${ }^{65}$ Likewise, higher rate of persistent bacteraemia was shown among patients affected with cefepime-SDD isolates who were treated with cefepime. ${ }^{62}$ However, only $16 \%$ of patients received full-dose cefepime.

\section{Klebsiella Pneumoniae Carbapenemase (KPC)-Producing Enterobacterales}

Recommentations are depicted in Figure 2, panel A.4. Ceftazidime-avibactam (2.5g LD followed by $2.5 \mathrm{~g}$ q $8 \mathrm{~h}$ over $8 \mathrm{~h} \mathrm{CI}$ ) is recommended as first-line therapy for the management of BSIs, cIAIs, and cUTIs caused by KPCproducing Enterobacterales. Meropenem-vaborbactam ( $2 \mathrm{~g} / 2 \mathrm{~g}$ q8h over $8 \mathrm{~h} \mathrm{CI}$ after $2 \mathrm{~g} / 2 \mathrm{~g}$ LD), imipenem-relebactam $(0.5 \mathrm{~g} / 0.25 \mathrm{~g}$ q6h over $3 \mathrm{~h})$, and cefiderocol $(2 \mathrm{~g}$ LD followed by $2 \mathrm{~g}$ q8h over $8 \mathrm{~h} \mathrm{CI}$ ) could be alternative options, and are listed in a hierarchical scale. A summary of scientific evidences is provided in Table 4. One prospective and eight retrospective observational studies support the role of ceftazidime-avibactam in the management of KPC-producing BSIs, cIAIs, and cUTIs in settings with an ICU admission up to $60 \% .{ }^{67-75}$ Van Duin et al assessed prospectively $\quad 137 \quad$ carbapenemase-producing Enterobacterales (CPE) infections (38 treated with ceftazidime-avibactam vs 99 with colistin-based regimens). ${ }^{67}$ Ceftazidime-avibactam showed a better adjusted probability of favourable outcome (64\%; $\mathrm{p}=0.0012)$, and a 3.5 -fold lower all-cause mortality rate than colistin-based regimens ( $8 \%$ vs $33 \% ; p=0.001$ ). Caston et al analysed retrospectively $31 \mathrm{CPE}$ infections among hematologic patients $(8$ treated with CAZ-AVI vs 23 treated with other antibiotic combinations, mainly carbapenems and aminoglycosides).$^{68}$ Patients treated with ceftazidime-avibactam showed significantly higher clinical cure rate ( $85.7 \%$ vs $34.8 \% ; \mathrm{p}=0.03)$. Tumbarello et al analysed 208 patients with KPC-producing Klebsiella pneumoniae BSIs (104 treated with ceftazidime-avibactam as second line therapy vs 104 treated with different rescue mono- or 


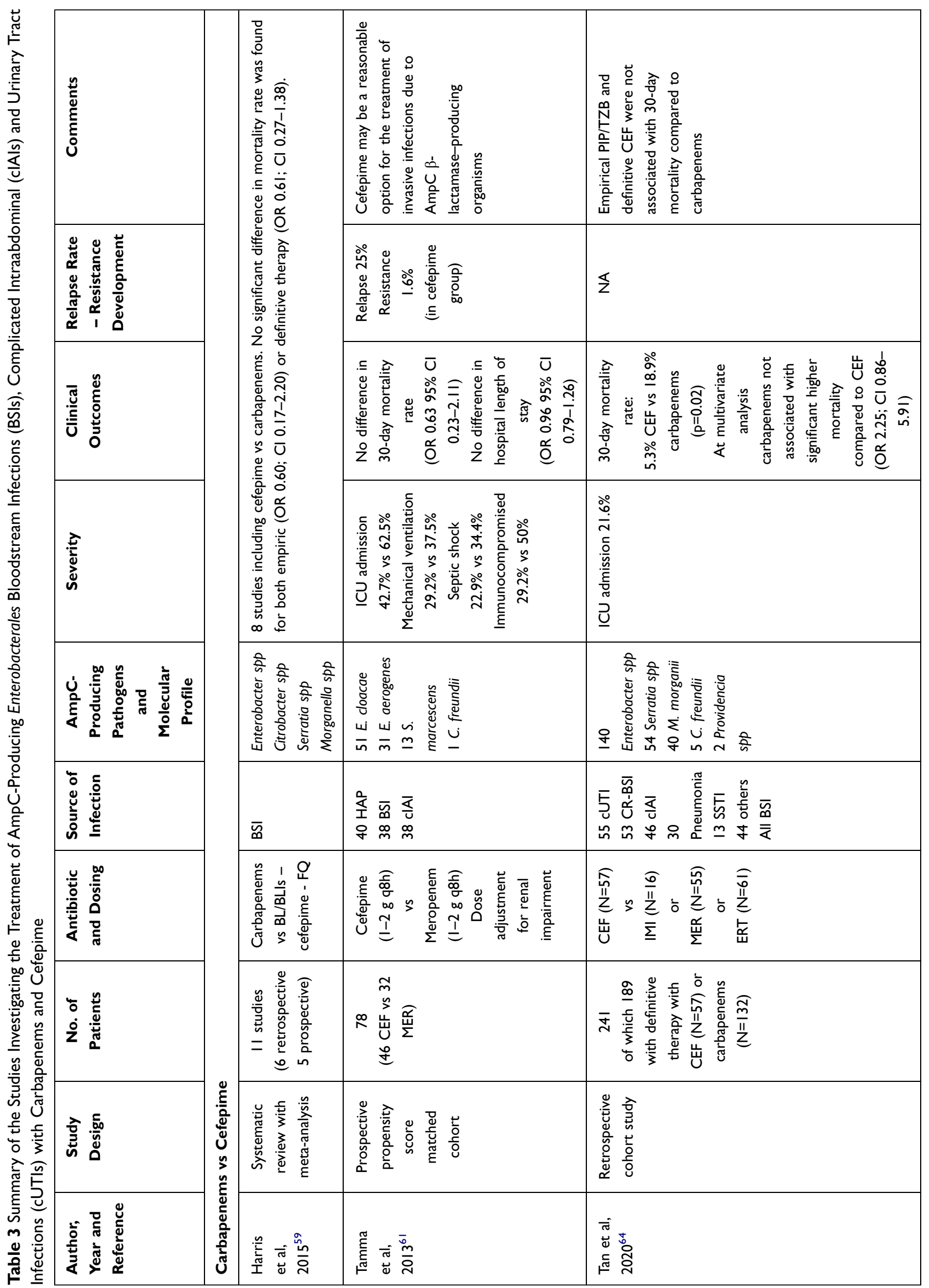




\begin{tabular}{|c|c|c|}
\hline 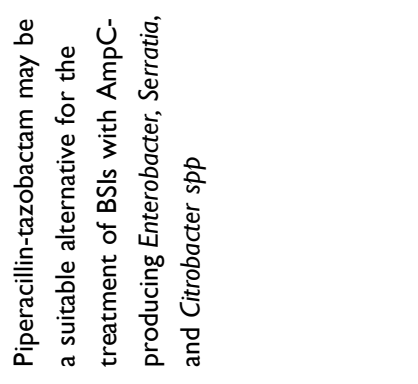 & 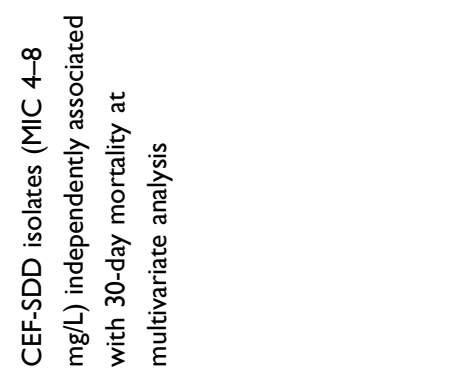 & 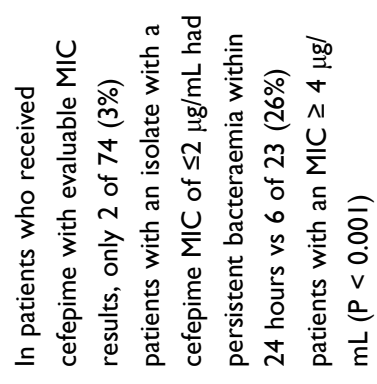 \\
\hline 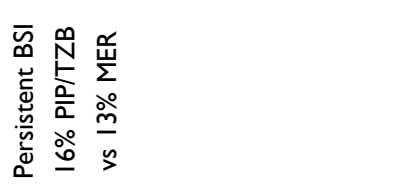 & $\S$ & 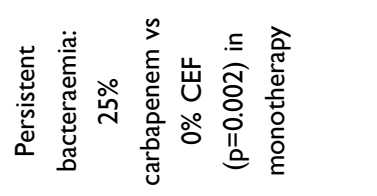 \\
\hline 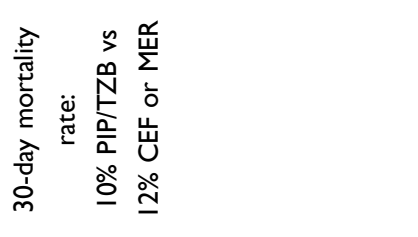 & 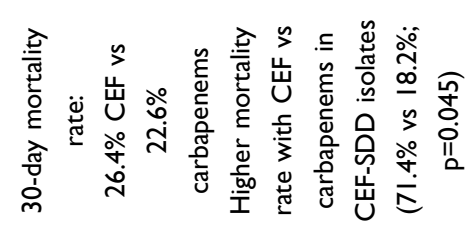 & 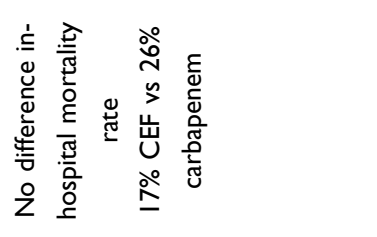 \\
\hline 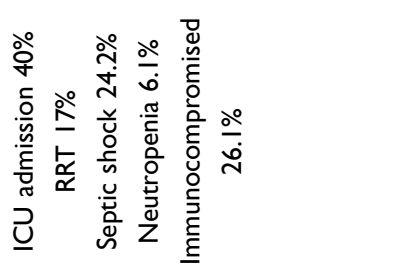 & 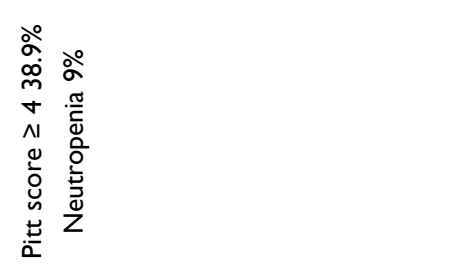 & 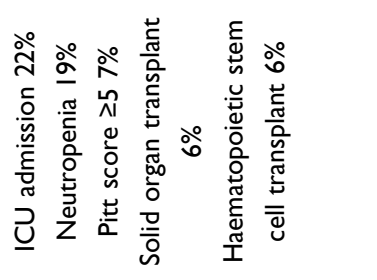 \\
\hline 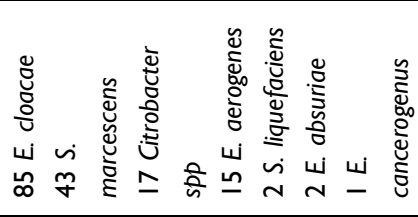 & 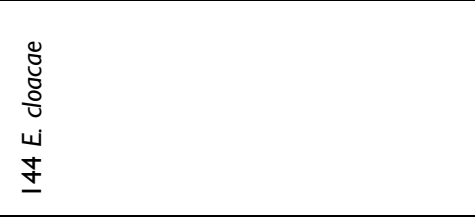 & 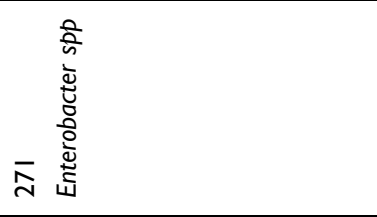 \\
\hline 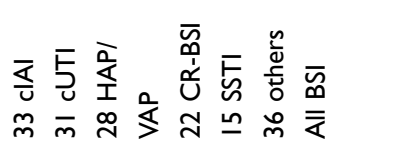 & 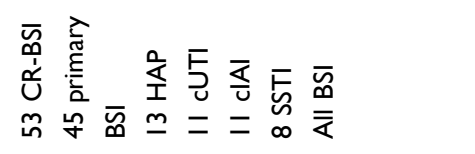 & 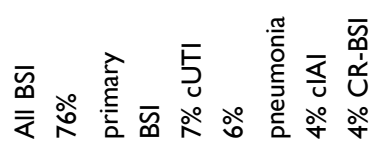 \\
\hline 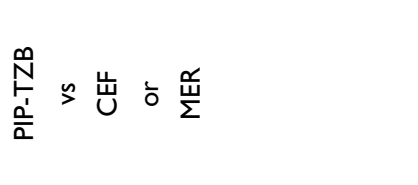 & 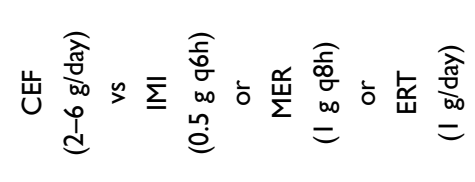 & 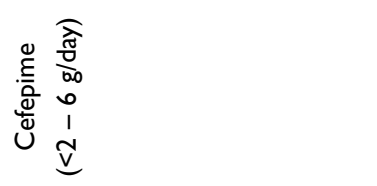 \\
\hline 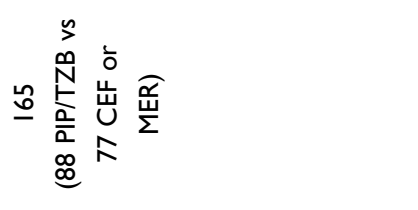 & 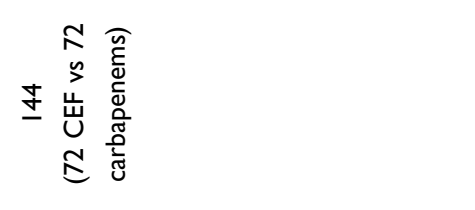 & 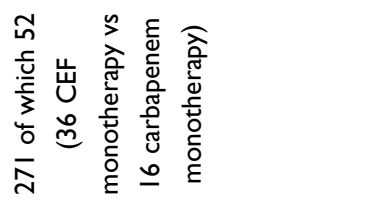 \\
\hline 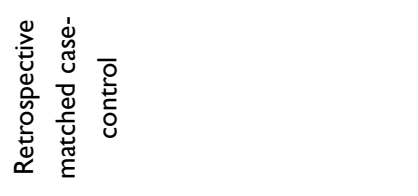 & 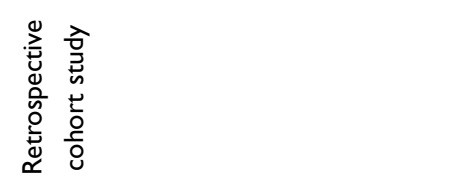 & 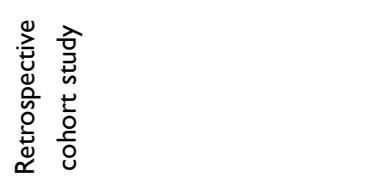 \\
\hline 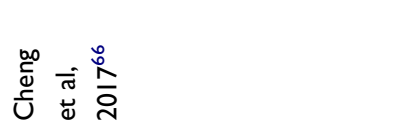 & 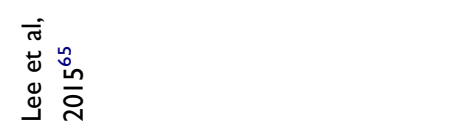 & 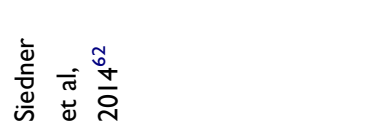 \\
\hline
\end{tabular}




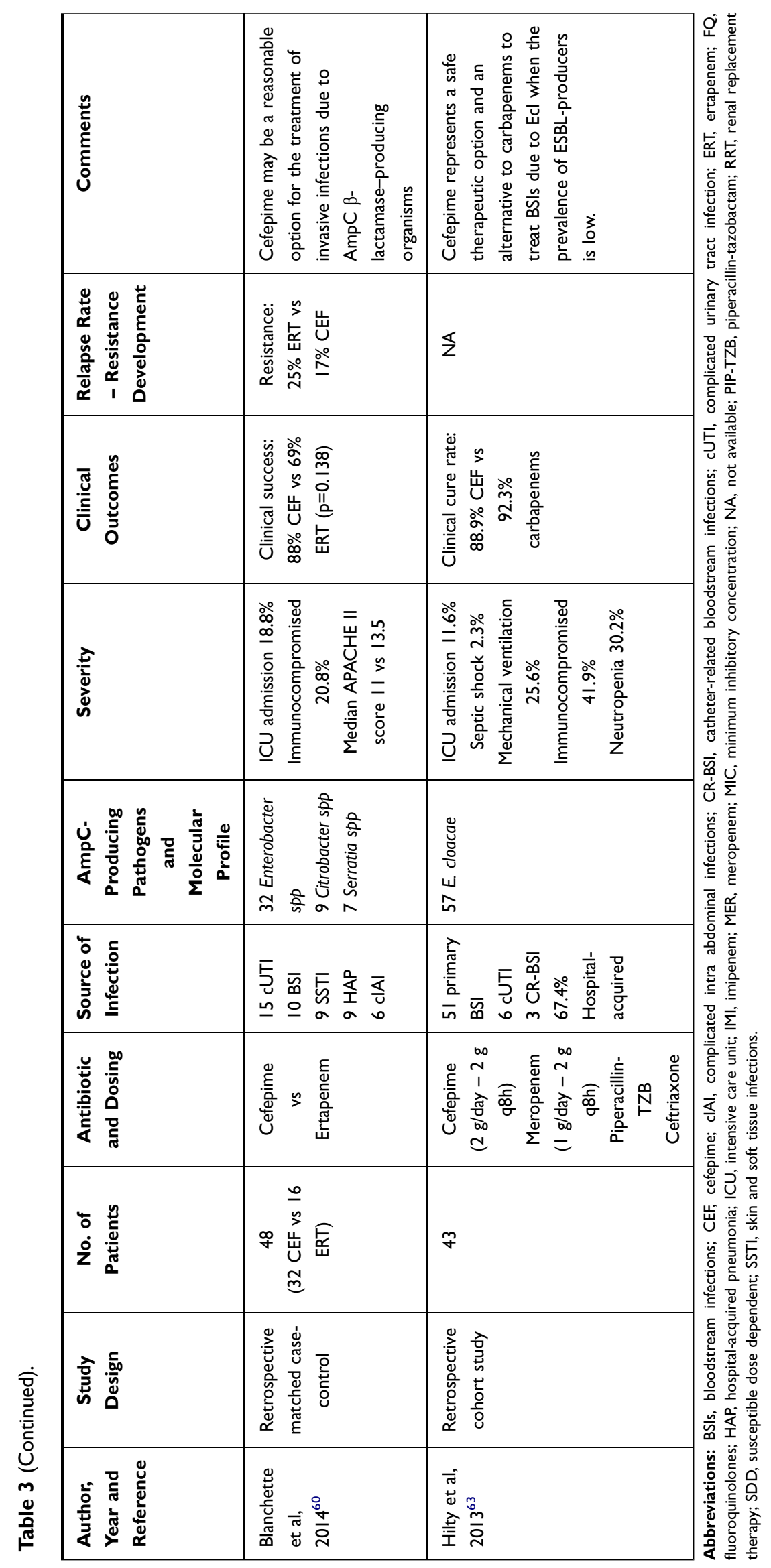




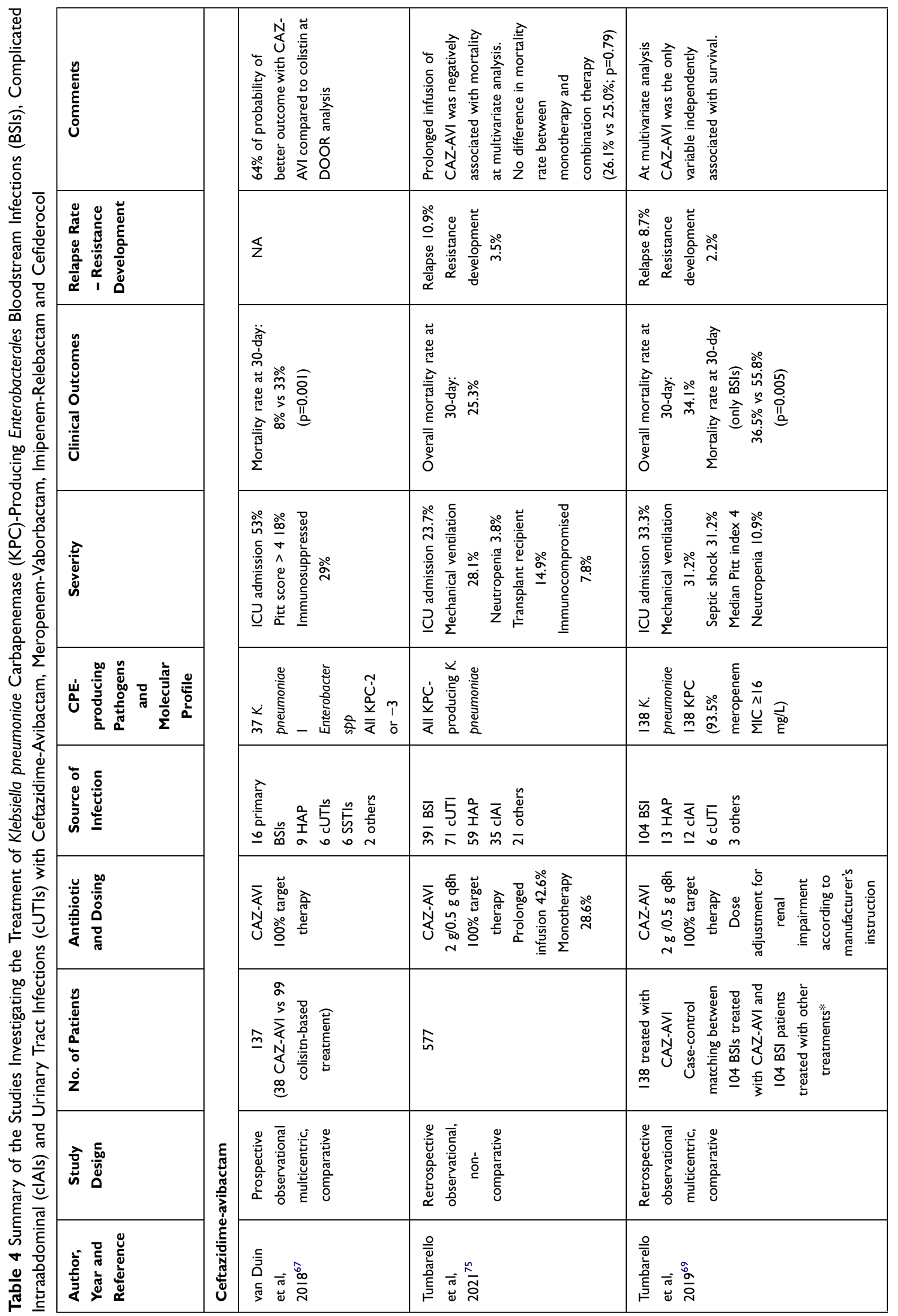




\begin{tabular}{|c|c|c|c|}
\hline 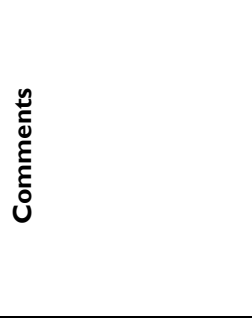 & 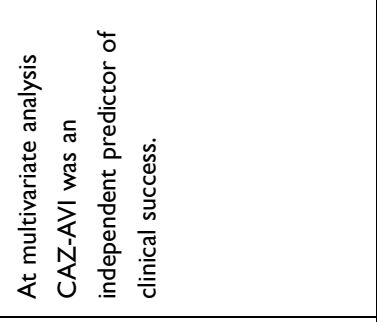 & 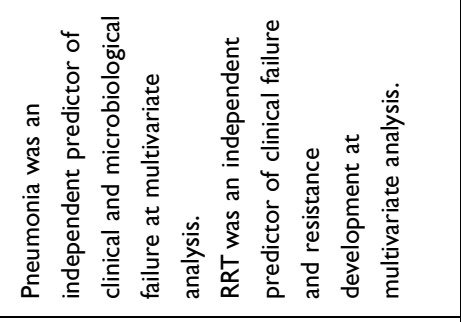 & 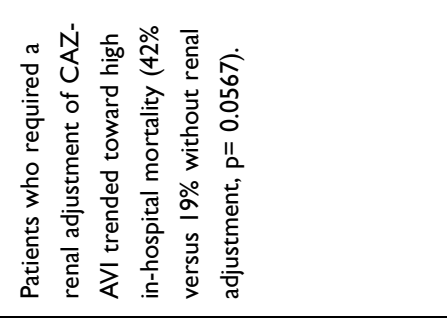 \\
\hline 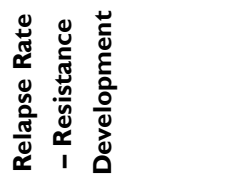 & 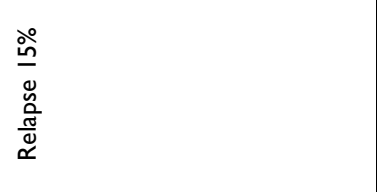 & 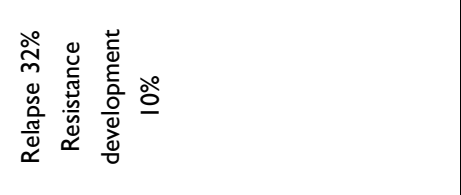 & 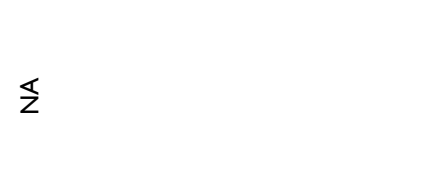 \\
\hline 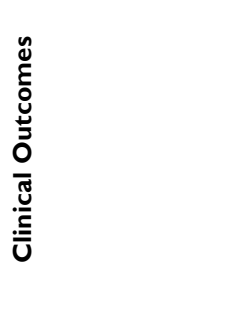 & 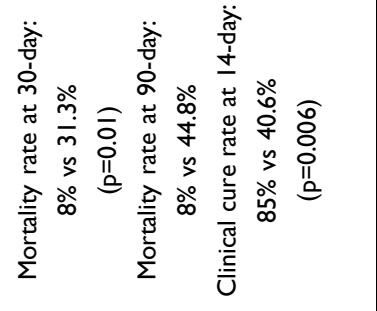 & 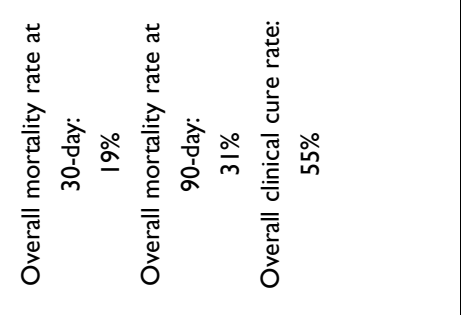 & 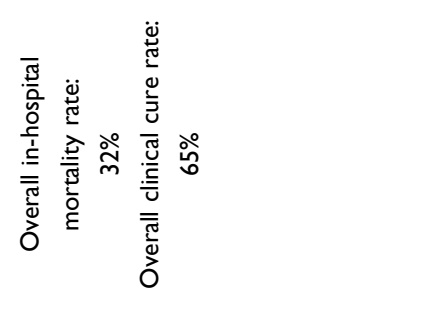 \\
\hline 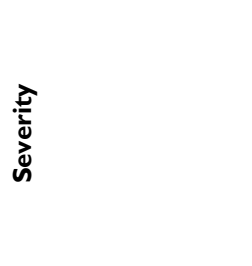 & 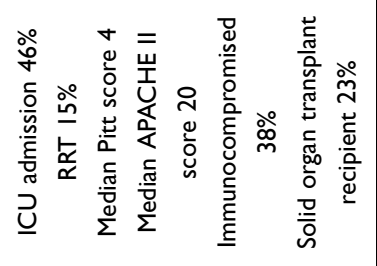 & 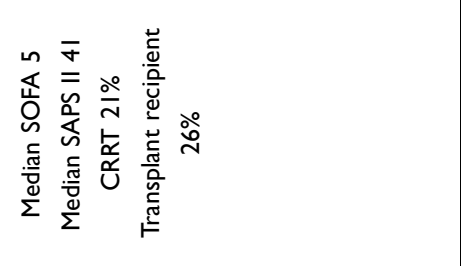 & 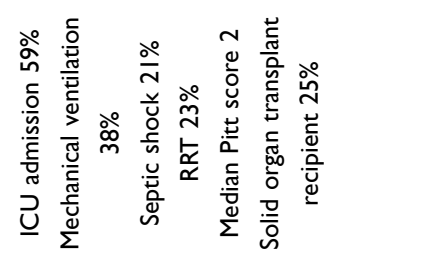 \\
\hline 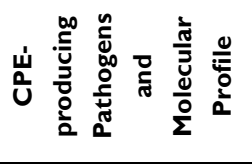 & 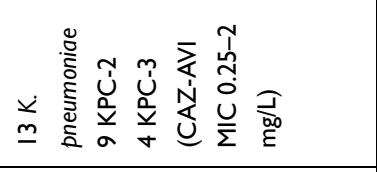 & 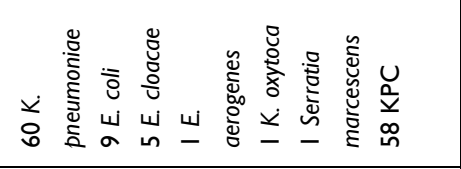 & 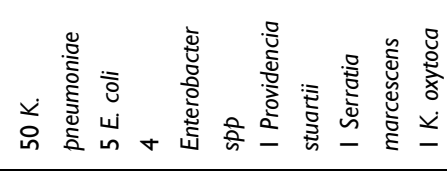 \\
\hline 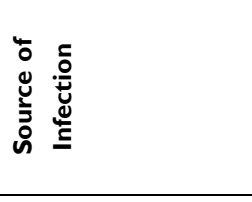 & 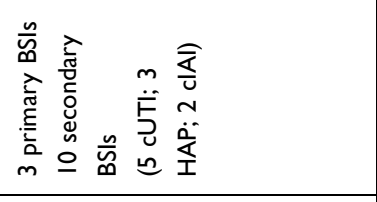 & 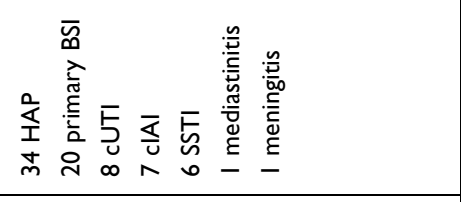 & 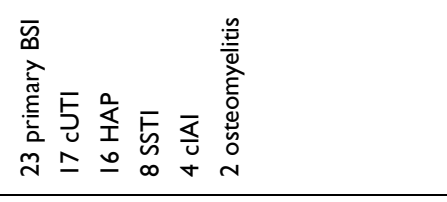 \\
\hline 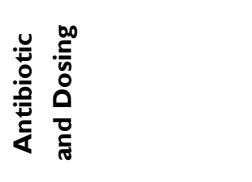 & 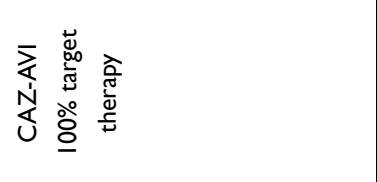 & 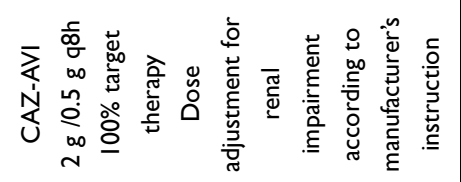 & 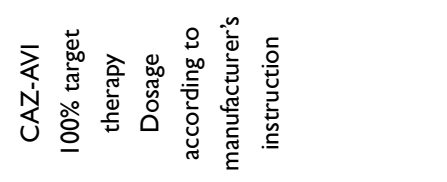 \\
\hline 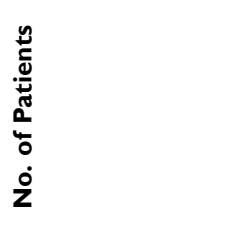 & 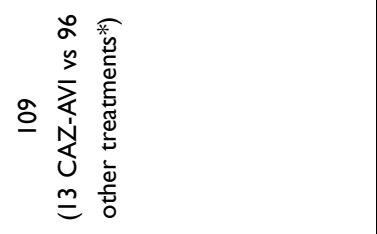 & $\kappa$ & 8 \\
\hline 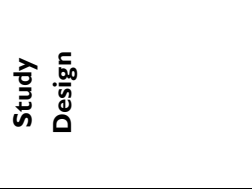 & 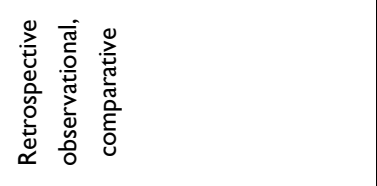 & 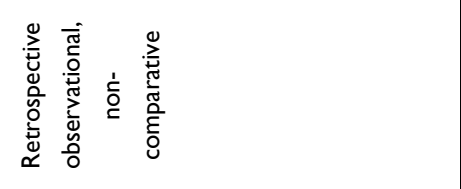 & 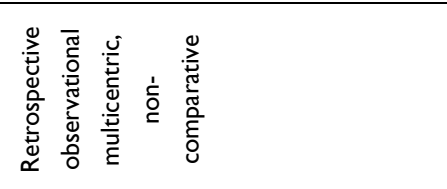 \\
\hline 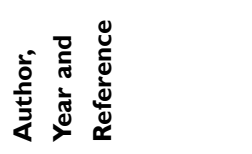 & 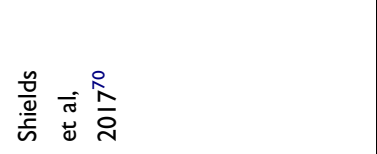 & 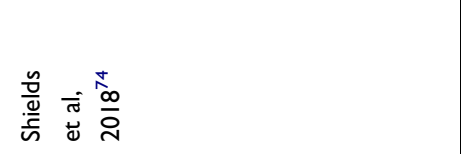 & 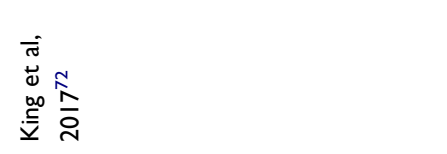 \\
\hline
\end{tabular}




\begin{tabular}{|c|c|c|}
\hline & 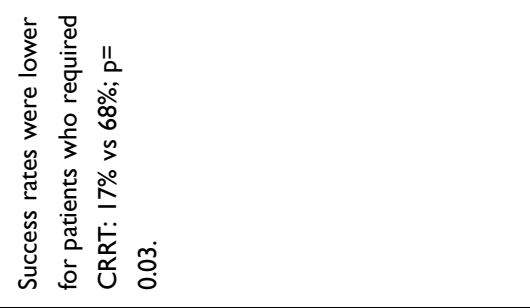 & 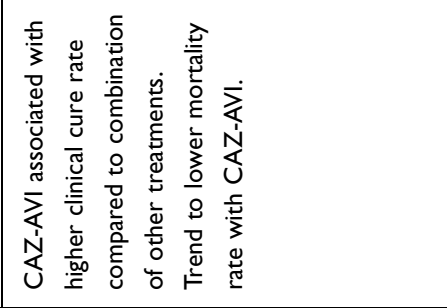 \\
\hline 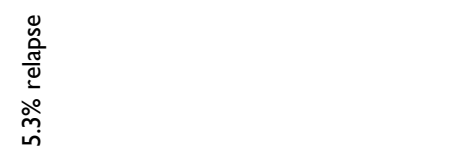 & 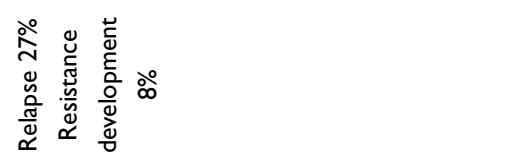 & $\overleftarrow{z}$ \\
\hline 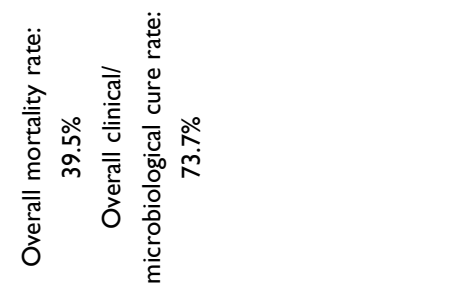 & 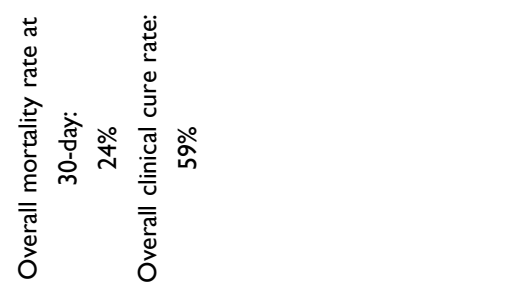 & 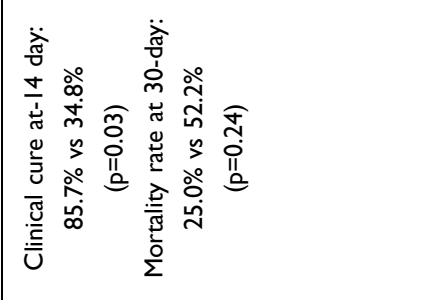 \\
\hline 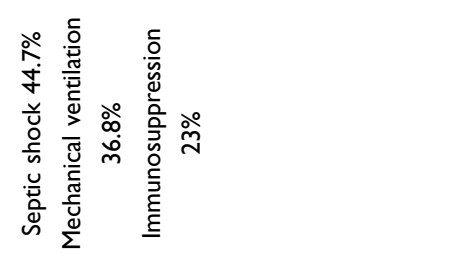 & 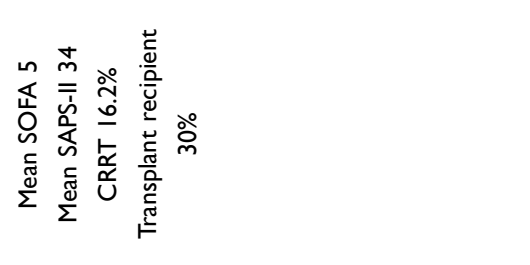 & 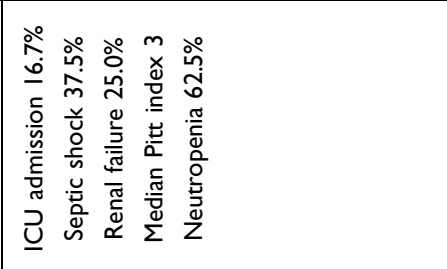 \\
\hline 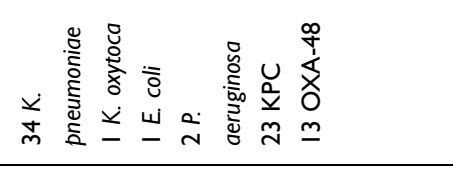 & 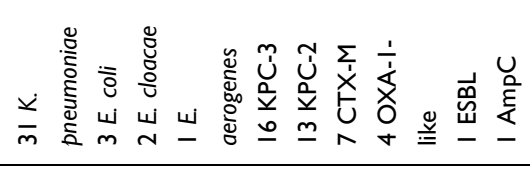 & 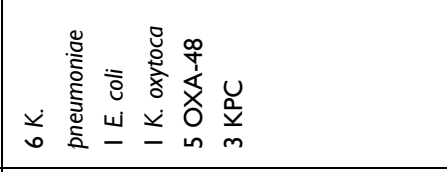 \\
\hline 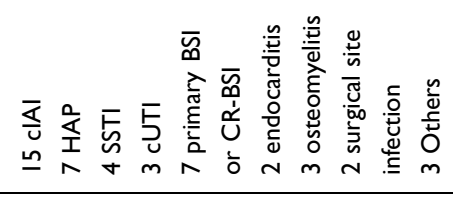 & 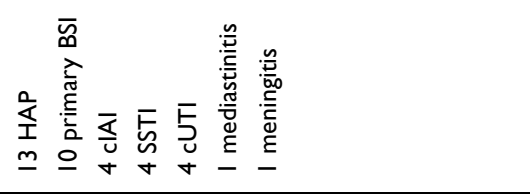 & 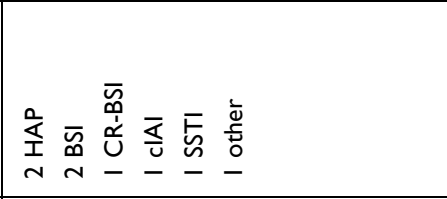 \\
\hline 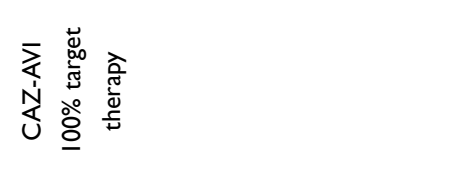 & 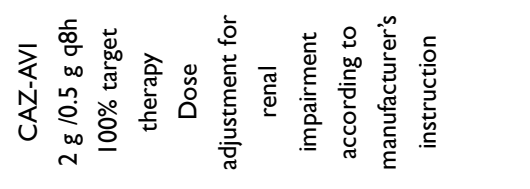 & 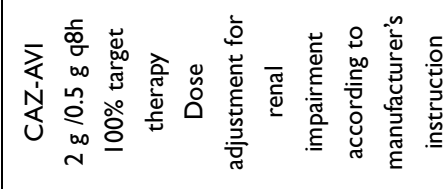 \\
\hline$\stackrel{\infty}{\infty}$ & $\hat{m}$ & 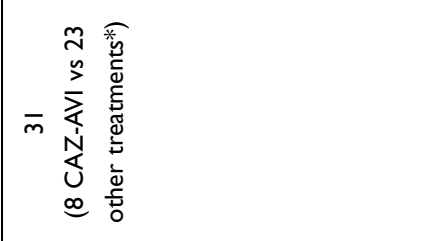 \\
\hline 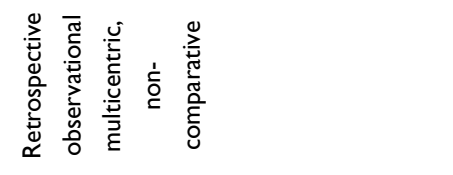 & 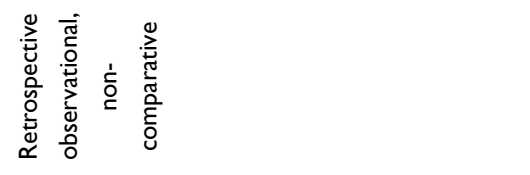 & 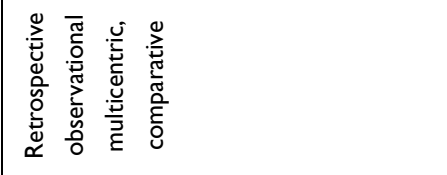 \\
\hline 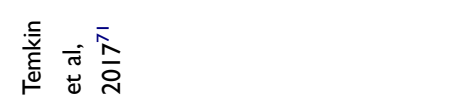 & 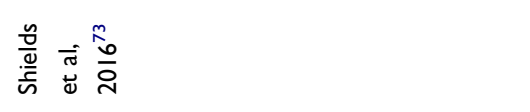 & 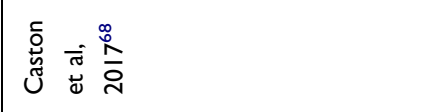 \\
\hline
\end{tabular}




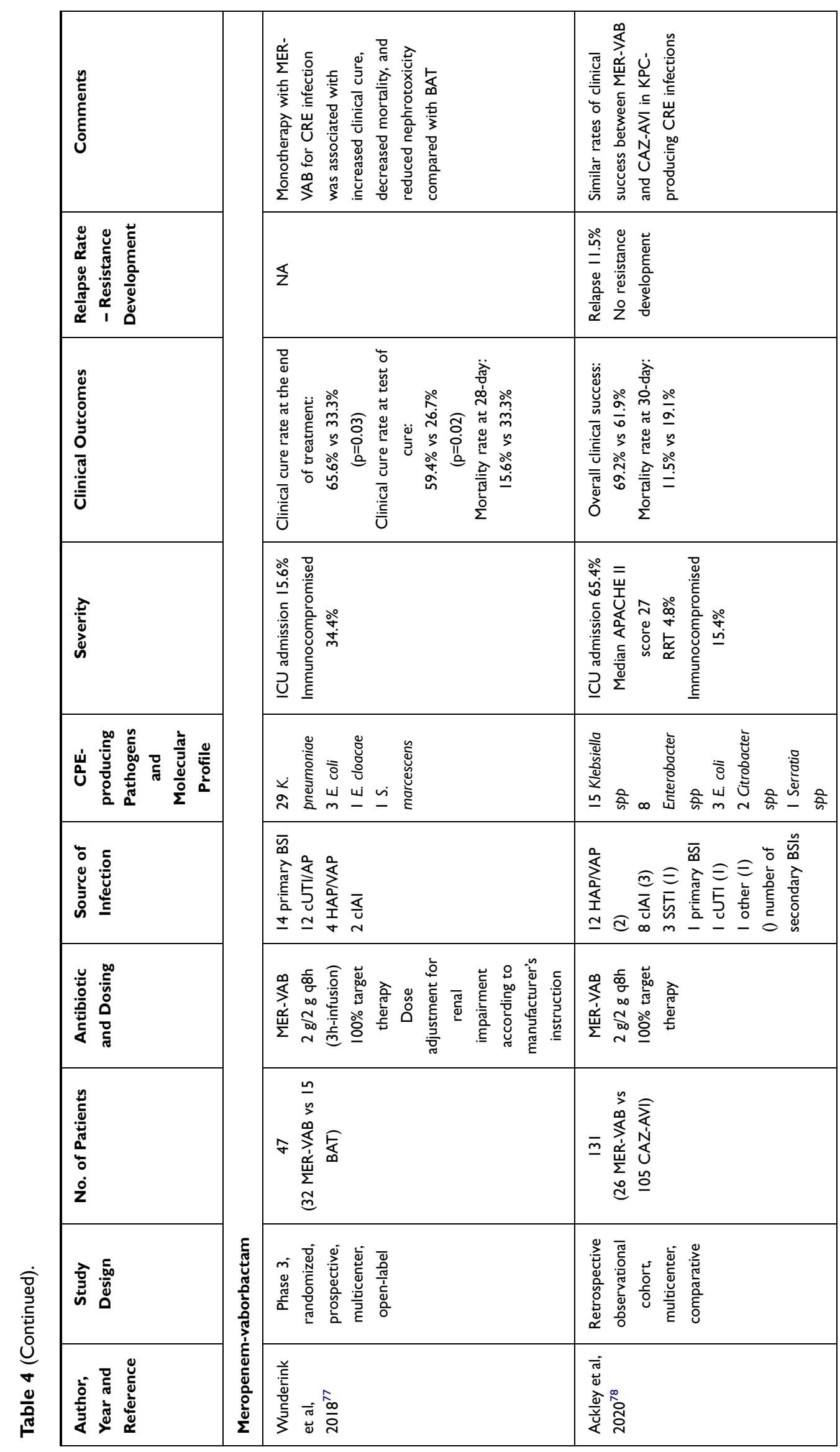




\begin{tabular}{|c|c|c|c|}
\hline & & & 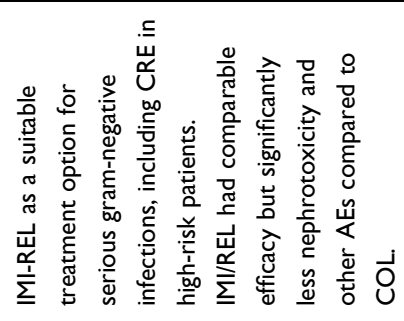 \\
\hline 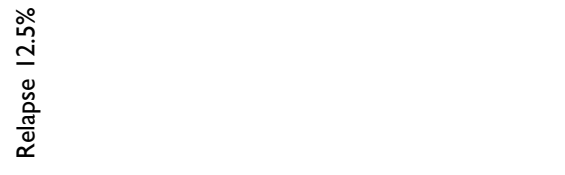 & 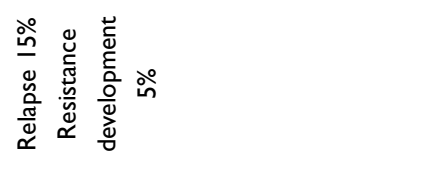 & & 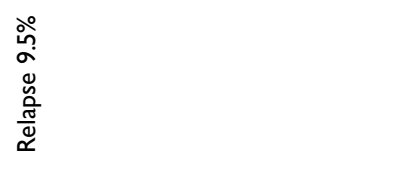 \\
\hline 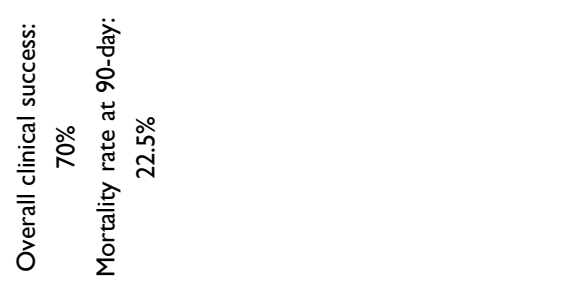 & 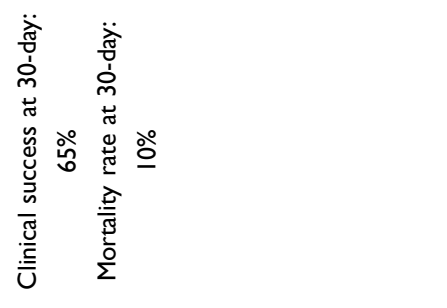 & & 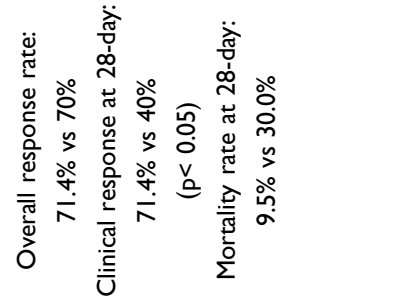 \\
\hline 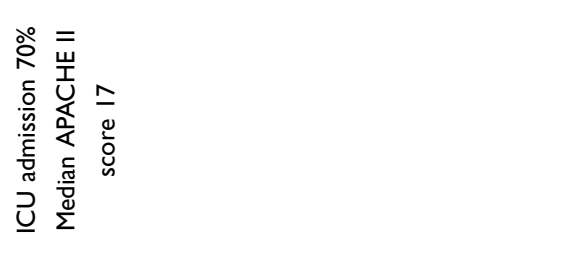 & 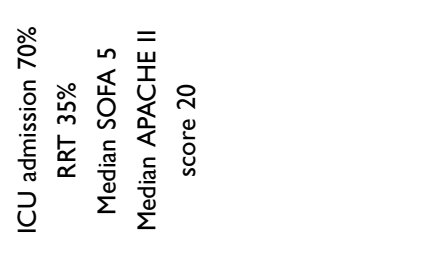 & & 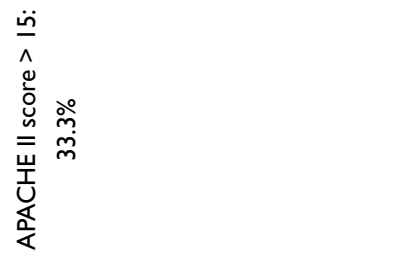 \\
\hline 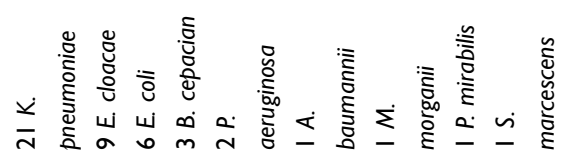 & 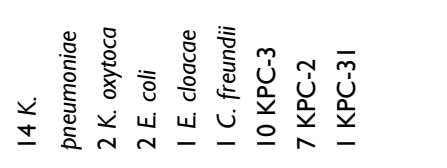 & & 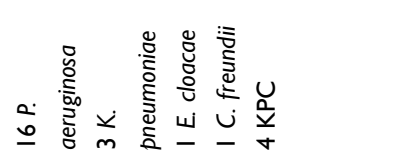 \\
\hline 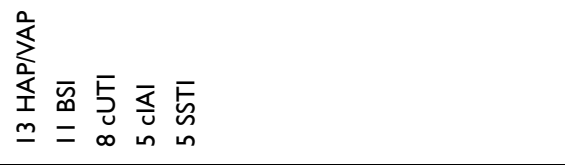 & 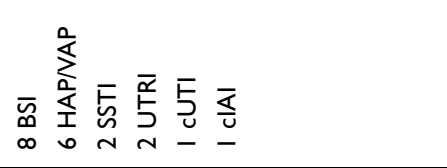 & & 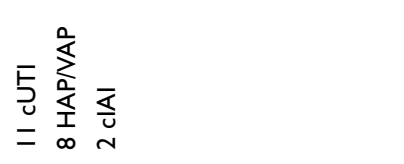 \\
\hline 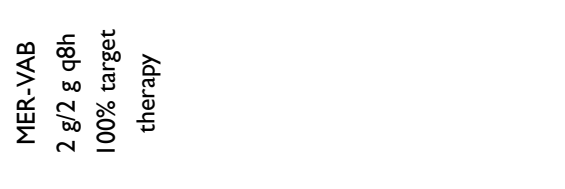 & 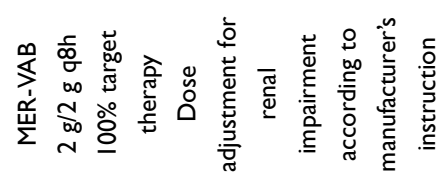 & & 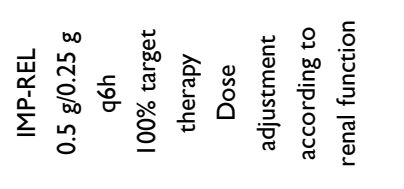 \\
\hline 우 & ని & & 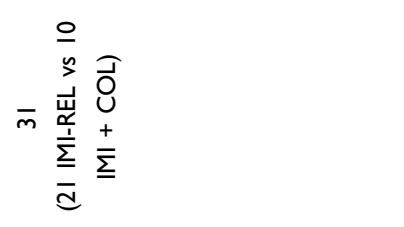 \\
\hline 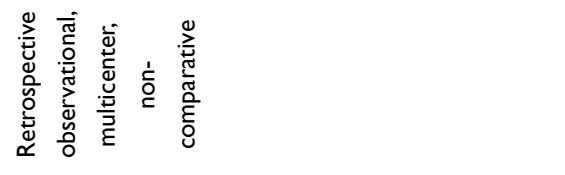 & 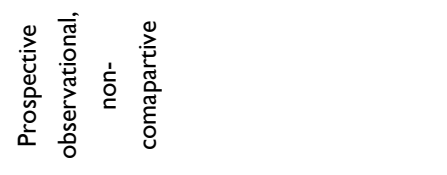 & 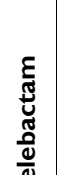 & 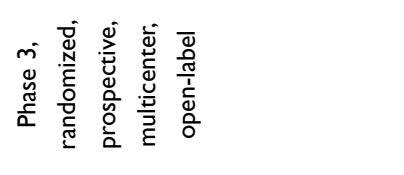 \\
\hline 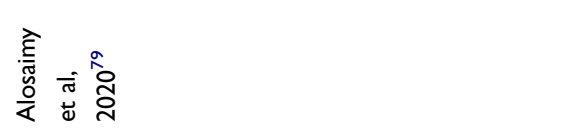 & 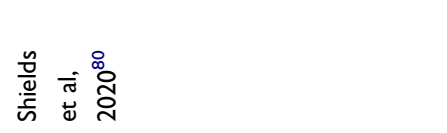 & 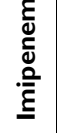 & 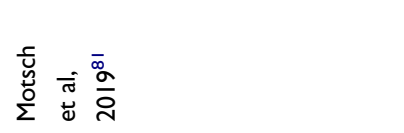 \\
\hline
\end{tabular}




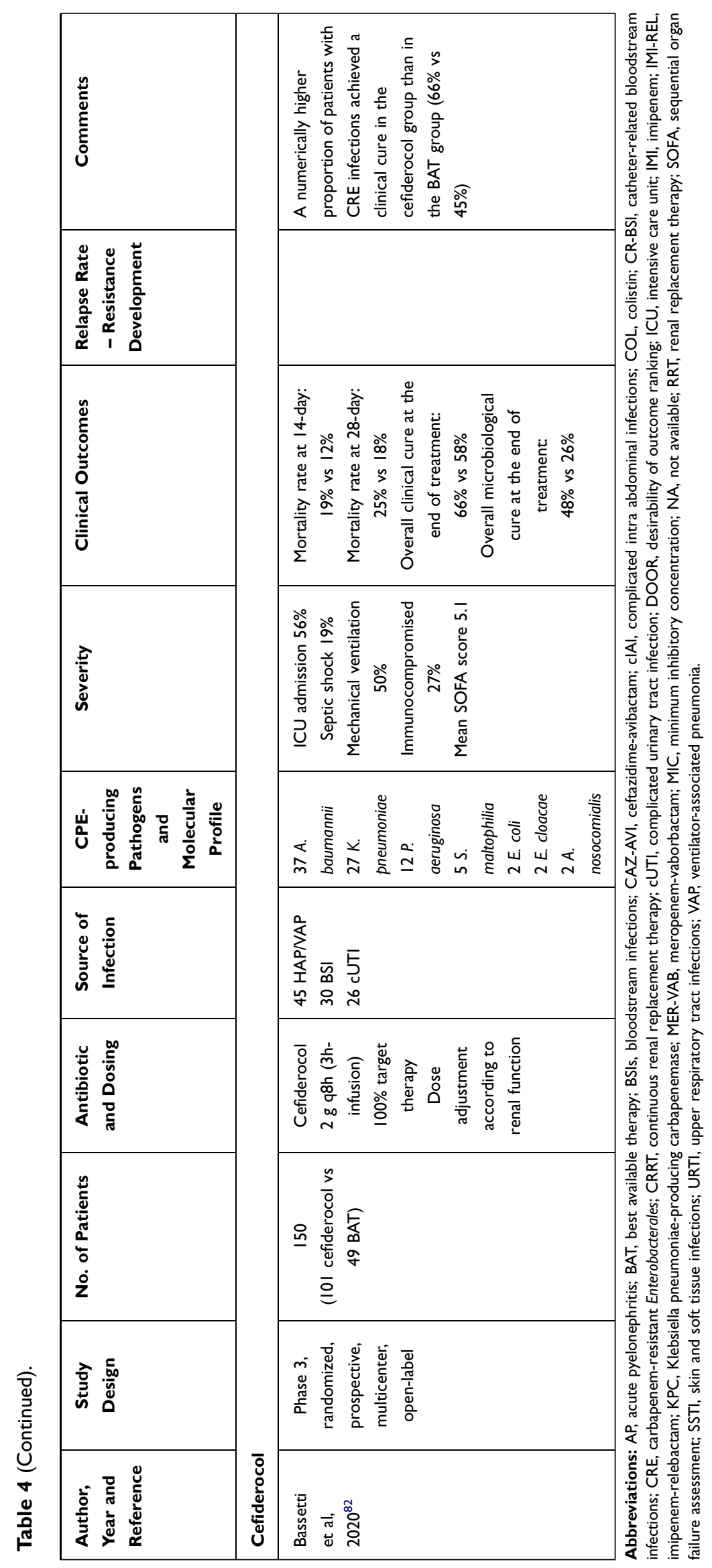


combo-treatments). ${ }^{69}$ Patients treated with ceftazidimeavibactam showed significantly lower 30-day mortality rate $(36.5 \%$ vs $55.8 \%$; $=0.005)$, and ceftazidime-avibactam was the only independent predictor of survival at multivariate analysis. Shields et al analysed 109 CPE infections, 13 of whom treated with ceftazidime-avibactam and the other 96 with other antimicrobials (mainly colistin, aminoglycosides and carbapenems). ${ }^{70}$ Patients receiving ceftazidime-avibactam showed significantly lower 30-day mortality rate ( $8 \%$ vs $31.3 \%$; $=0.01)$ and higher clinical success rate ( $85 \%$ vs $40.6 \%$; $=0.006)$. Very recently, Tumbarello et al analysed 577 patients with KPC-producing Klebsiella pneumoniae infections (67.8\% with BSIs) treated with ceftazidime-avibactam. ${ }^{75}$ No difference in mortality rate was found between ceftazidime-avibactam monotherapy vs combination therapy $(26.1 \%$ vs $25.0 \%$; $\mathrm{p}=0.79)$. Notably, ceftazidime-avibactam prolonged infusion resulted protective against mortality at multivariate analysis $(\mathrm{p}=0.006)$. In regard to meropenem-vaborbactam, it should be mentioned that vaborbactam was specifically developed to restore the activity of meropenem against KPCs. $^{76}$ A phase III RCT (TANGO II) assessed 47 patients affected by KPC-producing Enterobacterales infections, 32 of whom were treated with meropenemvaborbactam and the other 15 with best-available therapy (including mono/combination therapy with colistin, carbapenems, aminoglycosides, tigecycline, or ceftazidime-avibactam alone). Meropenem-vaborbactam showed better clinical cure rate $(65.6 \%$ vs $33.3 \% ; \mathrm{p}=0.03)$ and a trend toward lower mortality rate $(15.6 \%$ vs $33.3 \% ; \mathrm{p}=0.20)$ compared to best available therapy ${ }^{77}$ However, it should be recognized that patients enrolled in this RCT required ICU admission only in $15.6 \%$ of cases. More attractive evidence for meropenem-vaborbactam as targeted therapy for KPC-producing Enterobacterales infections in critically ill patients came from observational studies, in which ICU admission ranged from $65.4 \%$ to $70 \%{ }^{78-80}$ Clinical cure rate ranged $65-70 \%$, and mortality rate 10-22.5\%. Relapse rate of CPE infections ranged 11.5$15 \%$, and in up to $5 \%$ of patients was reported resistance development to meropenem-vaborbactam. One retrospective study ${ }^{78}$ reported no significant difference between 26 patients receiving meropenem-vaborbactam and 105 receiving ceftazidime-avibactam in terms of clinical cure rate ( 69.2 vs $61.9 \%)$ and mortality rate (11.5 vs $19.1 \%)$. In regard to imipenem-relebactam, it's worth mentioning that relebactam was combined to imipenem-cilastatin in order to restore activity against carbapenemase producing
Enterobacterales and Pseudomonas aeruginosa. ${ }^{76}$ In a phase III RCT of patients with severe Gram-negative infections, imipenem-relebactam demonstrated significantly better clinical cure rate compared to imipenem plus colistin $(71.4 \%$ vs $40 \%$; $<<0.05) .{ }^{81}$ However, infection by KPC-producing Enterobacterales was documented in only 4 out of the 21 patients enrolled in the imipenemrelebactam group. In regard to cefiderocol, in a phase III RCT 150 patients affected by carbapenem-resistant Gramnegative infections were randomized to cefiderocol $(n=101)$ or best available therapy (including combination of aminoglycoside, carbapenems, colistin, fosfomycin or tigecycline $)(\mathrm{n}=49) .{ }^{82}$ Clinical and microbiological cure rates between groups did not significantly differ. However, the number of documented KPC-producing Enterobacterales infections was quite limited.

\section{OXA-48-Producing Enterobacterales}

Recommendations are depicted in Figure 2, panel A.5. Ceftazidime-avibactam (2.5g LD followed by $2.5 \mathrm{~g}$ q $8 \mathrm{~h}$ over $8 \mathrm{~h} \mathrm{CI}$ ) is recommended as first-line therapy for the management of BSIs, cIAIs, and cUTIs caused by OXA48 and OXA-48-like-producing Enterobacterales. (avibactam inhibits OXA-48, and ceftazidime is stable to this enzyme) ${ }^{83}$ Cefiderocol ( $2 \mathrm{~g}$ LD followed by $2 \mathrm{~g}$ q8h over $8 \mathrm{~h} \mathrm{CI}$ ) could be an alternative option. A summary of the studies evaluating the efficacy of ceftazidime-avibactam and cefiderocol in this setting is provided in Table 5. Alraddadi et $\mathrm{al}^{84}$ compared retrospectively 10 patients treated with ceftazidime-avibactam with 28 treated with other mono- or combo-therapy (colistin, carbapenems, aminoglycosides, tigecycline, quinolone, cotrimoxazole, and aztreonam) for the management of CPE. After restricting analysis to OXA-48 infections, no difference in clinical cure $(75 \%$ vs $40 \% ; \mathrm{p}=0.21)$ and in mortality rate $(37.5 \%$ vs $50 \%$; $\mathrm{p}=0.69)$ were reported. In two observational studies concerning the treatment with ceftazidimeavibactam of infections caused by OXA-48-producing Enterobacterales, ${ }^{85,86}$ the clinical cure rate and mortality rate were similar to those found in other studies where it was used for the treatment of KPC infections [59-62]. Conversely, in one retrospective study assessing ceftazidime-avibactam as salvage therapy for infections caused by carbapenem-resistant organisms,${ }^{71}$ among the 13 patients who were affected by OXA-48 infections a trend toward lower microbiological cure ( $25 \%$ vs $75 \%$; $\mathrm{p}=0.07$ ) and survival to hospital discharge $(22.7 \%$ vs $77.3 \%$; $\mathrm{p}=0.07$ ) compared to the 23 who had KPC infections was 


\begin{tabular}{|c|c|c|c|c|}
\hline 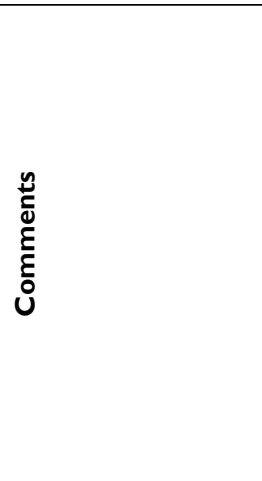 & & 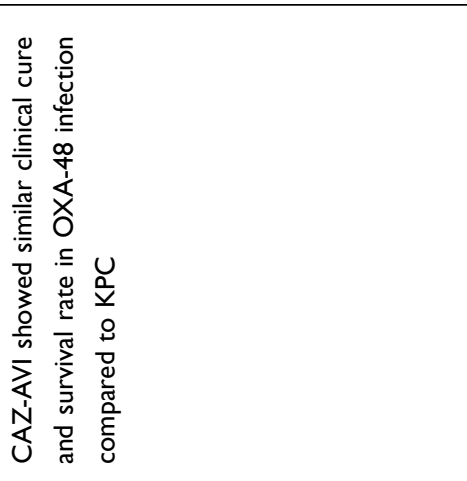 & 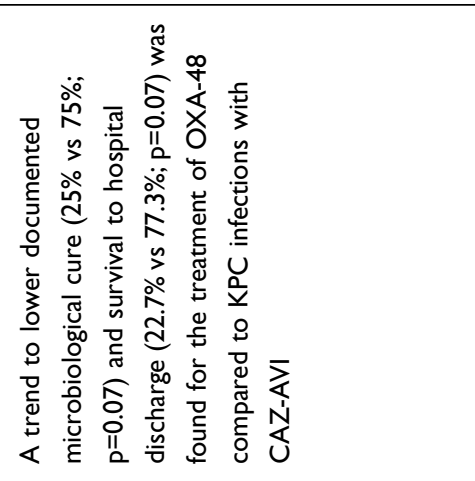 & 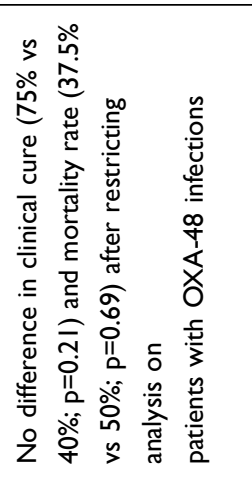 \\
\hline 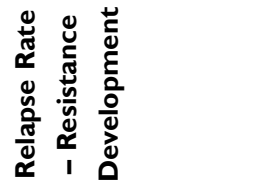 & & 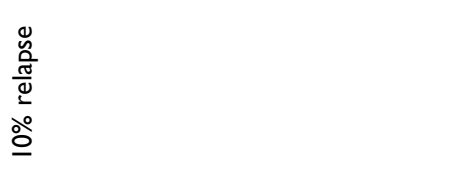 & 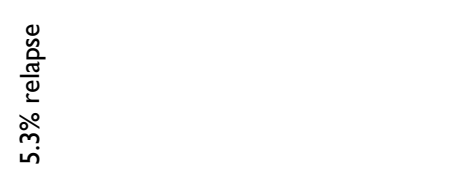 & 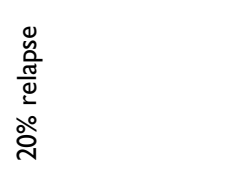 \\
\hline 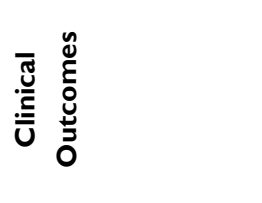 & & 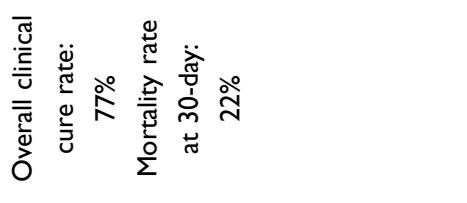 & 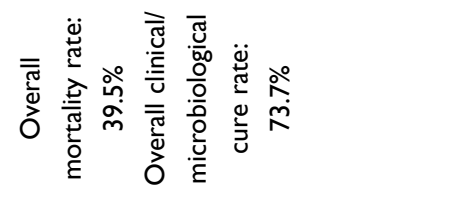 & 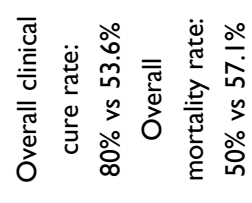 \\
\hline 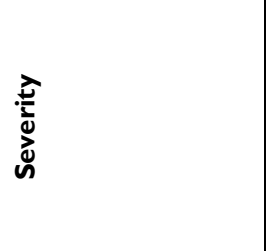 & & 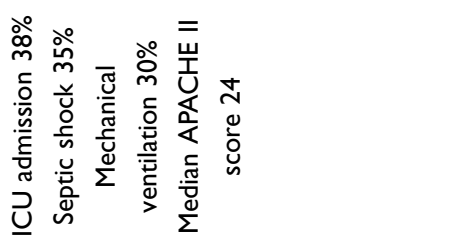 & 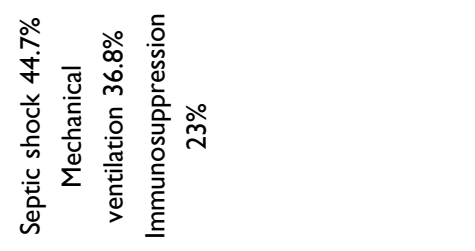 & 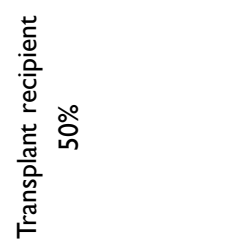 \\
\hline 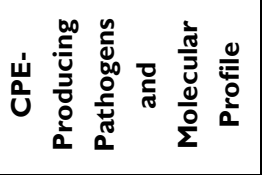 & & 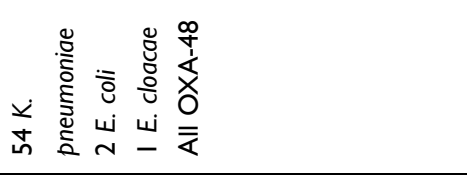 & 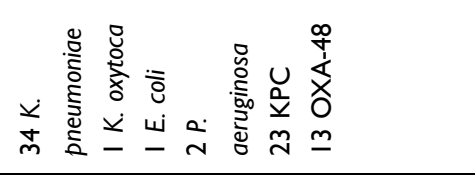 & 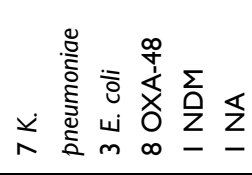 \\
\hline 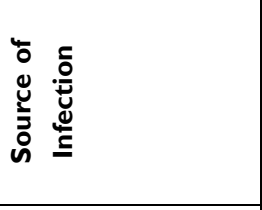 & & 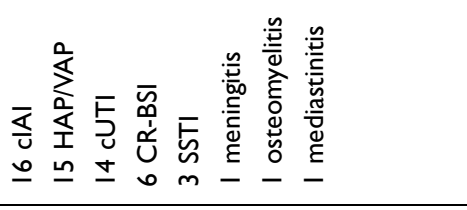 & 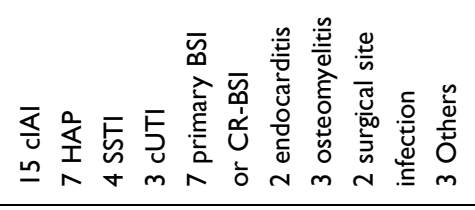 & 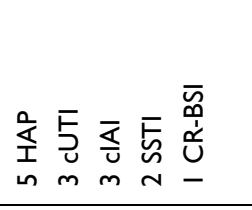 \\
\hline 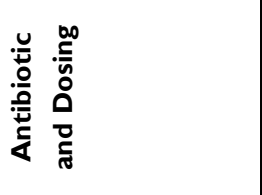 & & 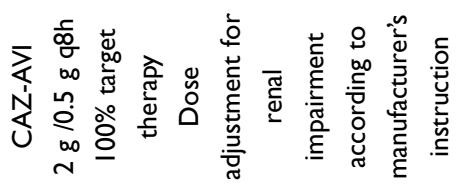 & 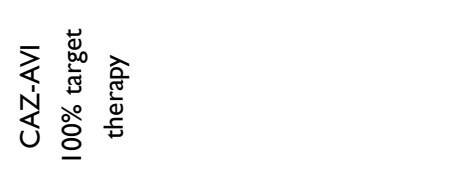 & 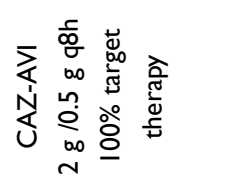 \\
\hline 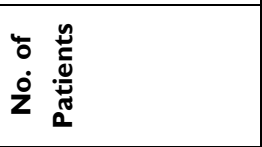 & & in & $\stackrel{\infty}{m}$ & 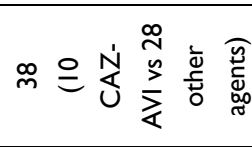 \\
\hline 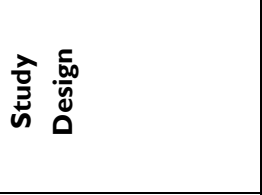 & 胥 & 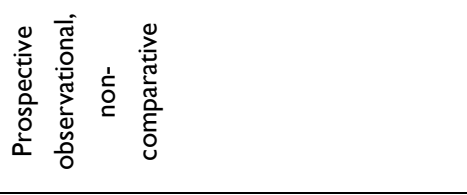 & 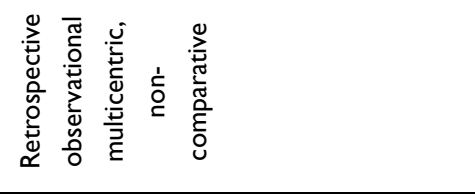 & 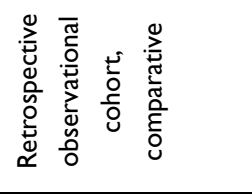 \\
\hline 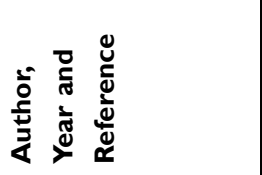 & 选 & 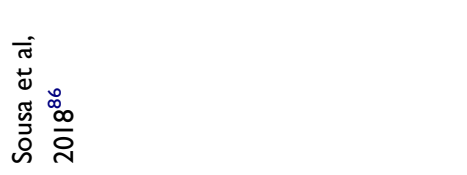 & 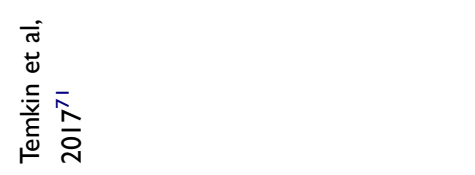 & 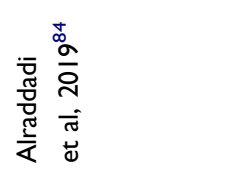 \\
\hline
\end{tabular}




\begin{tabular}{|c|c|c|c|c|c|c|}
\hline 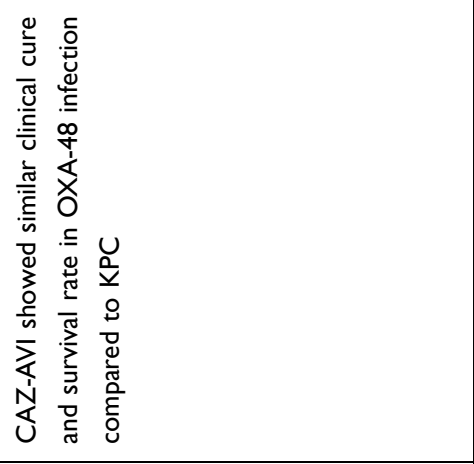 & & & $\stackrel{\partial}{\partial}$ & & 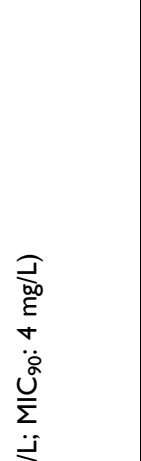 & \\
\hline 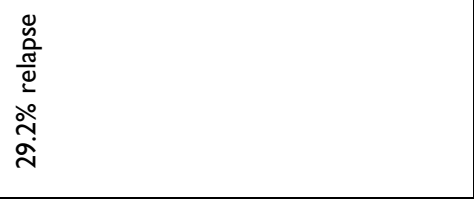 & & $\begin{array}{l}心 \\
\stackrel{0}{0} \\
\frac{\omega}{0} \\
\stackrel{0}{z}\end{array}$ & 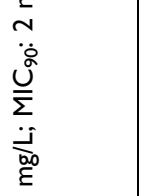 & 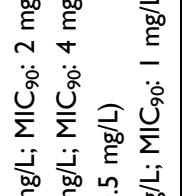 & 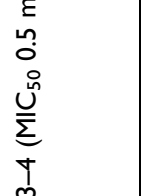 & \\
\hline 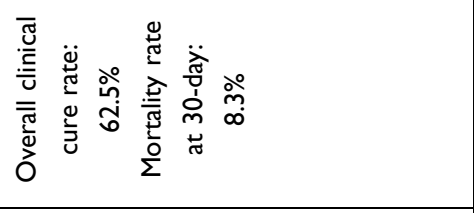 & & 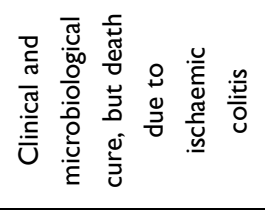 & 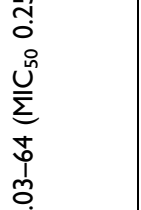 & 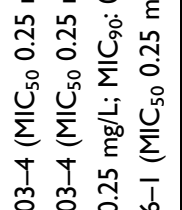 & 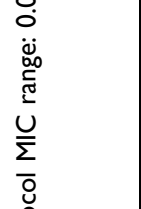 & 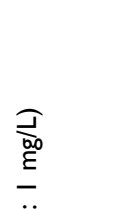 \\
\hline 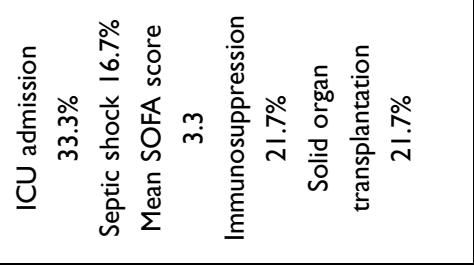 & & 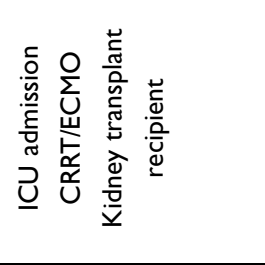 & 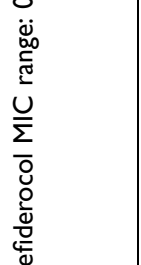 & 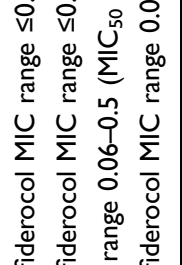 & 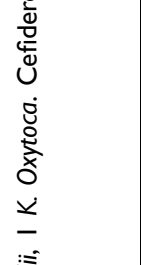 & 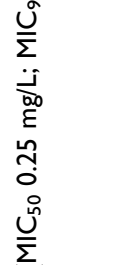 \\
\hline 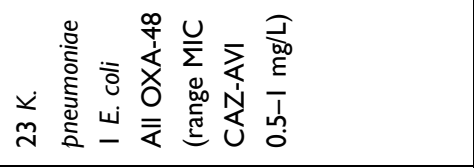 & & 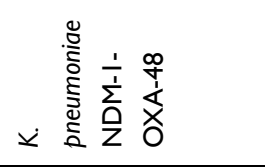 & 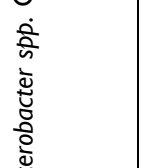 & 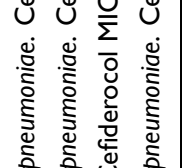 & 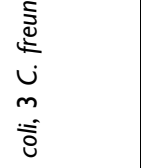 & 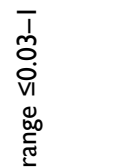 \\
\hline 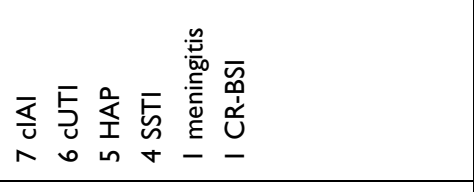 & & 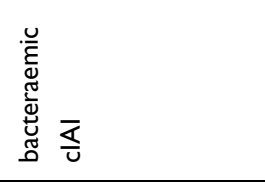 & 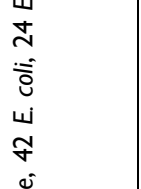 & 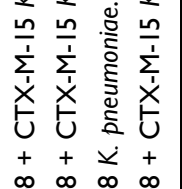 & 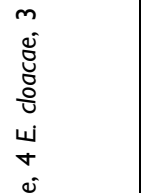 & 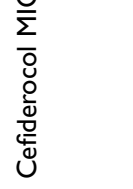 \\
\hline 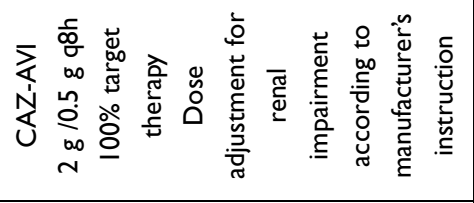 & & 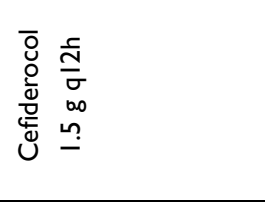 & 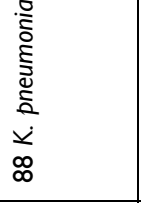 & 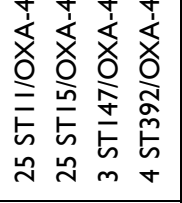 & 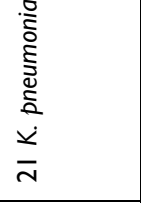 & 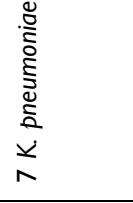 \\
\hline$\stackrel{ \pm}{\sim}$ & & - & 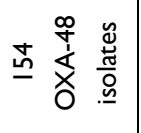 & 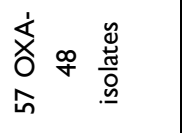 & 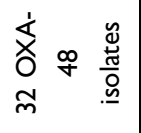 & 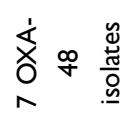 \\
\hline 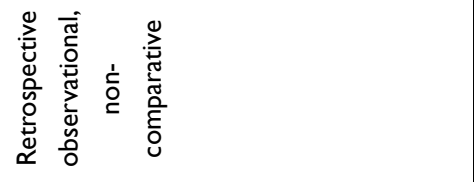 & & 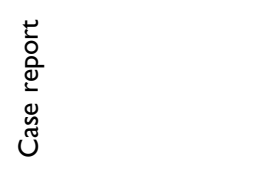 & 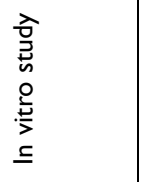 & 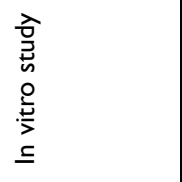 & 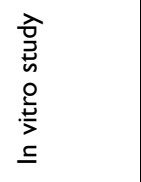 & 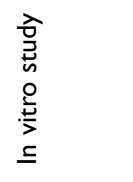 \\
\hline 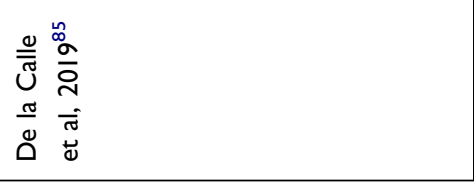 & 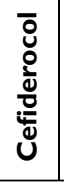 & 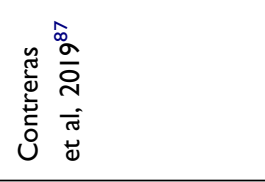 & 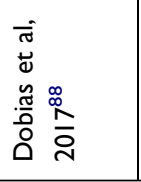 & 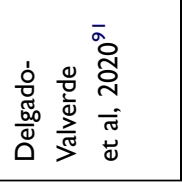 & 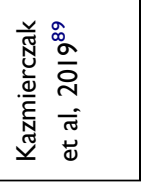 & 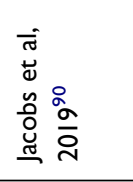 \\
\hline
\end{tabular}


found. In regard to cefiderocol, there is only one case $\mathrm{e}^{87}$ that reported its use for the management of a secondary BSI caused by carbapenem-resistant $K$. pneumoniae coproducing OXA-48-like and New Delhi metallo-beta-lactamase-1 (NDM-1). Microbiological cure was proven, and the patient died because of an ischaemic colitis secondary to Clostridium difficile infection. Besides, several in vitro studies $^{88-91}$ support the good activity of cefiderocol against OXA-48 producing Enterobacterales, with $\mathrm{MIC}_{50}$ and $\mathrm{MIC}_{90}$ ranging $0.25-0.5 \mathrm{mg} / \mathrm{L}$, and $0.5-4 \mathrm{mg} / \mathrm{L}$, respectively.

\section{Metallo-Beta-Lactamase (MBL)-Positive Enterobacterales}

Recommendations are depicted in Figure 2, panel A.6. Ceftazidime-avibactam (2.5g LD followed by $2.5 \mathrm{~g}$ q $8 \mathrm{~h}$ over $8 \mathrm{~h} \mathrm{CI}$ ) plus aztreonam ( $2 \mathrm{~g}$ q $8 \mathrm{~h}$ over $8 \mathrm{~h} \mathrm{CI}$ after $2 \mathrm{~g} \mathrm{LD}$ ) is the recommended first-line therapy for the management of BSIs, cIAIs, and cUTIs caused by New-Delhi metallo beta-lactamases (NDM)-producing Enterobacterales. Cefiderocol (2g $\mathrm{LD}$ followed by $2 \mathrm{~g}$ q $8 \mathrm{~h}$ over $8 \mathrm{~h} \mathrm{CI}$ ) is recommended for the treatment of infections caused by Verona Integron-encoded metallo-beta-lactamase (VIM)-producing and/or imipenemase (IMP)-producing Enterobacterales. Fosfomycin (6g LD followed by $16 \mathrm{~g} \mathrm{CI}$ ) plus high-dose meropenem (1.5$2 \mathrm{~g}$ q6h over $6 \mathrm{~h} \mathrm{CI}$ after $2 \mathrm{~g} \mathrm{LD}$ ) could be an alternative option for both NDM-producing and VIM-IMP-producing Enterobacterales. MBLs are associated with extremelydrug-resistant (XDR) phenotypes, as they may hydrolyse the vast majority of currently available beta-lactams. ${ }^{92} \mathrm{~A}$ summary of the studies evaluating the efficacy of these antibiotics in the setting is provided in Table 6 . One prospective observational study, two case series and five case reports suggest the efficacy of the combination therapy ceftazidime-avibactam plus aztreonam in the treatment of critically ill patients affected by NDM infections (mainly expressed by Klebsiella pneumoniae). ${ }^{93-100}$ Falcone et al ${ }^{93}$ compared in a prospective observational study 52 patients receiving the combination ceftazidime-avibactam plus aztreonam with 50 subjects receiving other active antibiotics in the management of BSIs due to NDM-producing and/or VIM-producing Enterobacterales. Patients treated with ceftazidime-avibactam plus aztreonam showed significantly lower mortality rate $(19.2 \%$ vs $44 \% ; \mathrm{p}=0.007)$ and clinical failure rate $(25 \%$ vs $52 \% ; \mathrm{p}=0.005)$. Shaw et $\mathrm{al}^{94}$ reported a case series of ceftazidime-avibactam plus aztreonam for the treatment of an outbreak caused by NDM-1/OXA-48-producing Klebsiella pneumoniae strain. Among 10 treated patients, 4 were solid organ transplant recipients, and half had bacteraemic infections (including cUTIs and cIAIs). Overall clinical cure rate at 30 -day was $60 \%$, and three patients died. Cairns et al ${ }^{101}$ reported a case series of four immunocompromised patients affected by IMP-4-producing Enterobacter cloacae infections successfully treated with ceftazidime-avibactam plus aztreonam, and relapse occurred only in one case. In regard to cefiderocol, only one case reported its role in the management of a secondary BSI caused by carbapenem-resistant $K$. pneumoniae co-producing NDM-1 and OXA-48-like betalactamases. ${ }^{87}$ However, several in vitro studies support the good activity of cefiderocol against NDM-producing Enterobacterales ( $\mathrm{MIC}_{50}: 1-4 \mathrm{mg} / \mathrm{L}$ and $\mathrm{MIC}_{90}: 4-8 \mathrm{mg} / \mathrm{L}$, susceptibility rate of 41-72.1\%), and IMP-VIM-positive isolates $\left(\mathrm{MIC}_{50} 1 \mathrm{mg} / \mathrm{L}, \mathrm{MIC}_{90} 4 \mathrm{mg} / \mathrm{L}\right.$, susceptibility rate of 80.9-95.7\%). ${ }^{88,89,102}$ In regard to the combination of fosfomycin with high-dose meropenem, only one case documented the efficacy of this combo in a kidney transplant recipient affected by bacteraemic cUTI due to NDM-1-producing Morganella morganii. ${ }^{103}$ An in vitro study showed the synergistic effect of this combination against 10 NDM-producing Klebsiella pneumoniae strains (including five isolates coproducing OXA-48 carbapenemases). ${ }^{104}$

\section{Overview of Recommendations}

The widespread diffusion of CRE isolates and the different genotypes of resistance of Enterobacterales requires careful attention for the right place in therapy of novel beta-lactams and the prompt adoption of strategies for sparing the broadest-spectrum antibiotics whenever possible. Ampicillin-sulbactam and ceftriaxone still represent the first-choice treatment of BSIs, cIAIs, or cUTIs caused by multi-susceptible Enterobacterales. Piperacillin-tazobactam should be recommended for the treatment of BSIs, cIAIs, or cUTIs caused by ESBL-producing Enterobacterales with an MIC $\leq 8 \mathrm{mg} / \mathrm{L}$, especially in low-risk patients. ${ }^{105}$ In this scenario, altered dosing strategies based on high-doses administered by CI may maximize clinical efficacy of piperacillin-tazobactam against ESBL isolates, and this approach has been recently suggested by the EUCAST as well. ${ }^{105}$ Ertapenem was not recommended for the treatment of ESBL-producing infections in critically ill patients, as some studies ${ }^{106,107}$ showed that among patients affected by septic shock treatment with ertapenem was associated with higher mortality rate compared to other carbapenems. Cefepime may represent an effective "carbapenem-sparing" strategy for the treatment of infections caused by AmpC-producing 


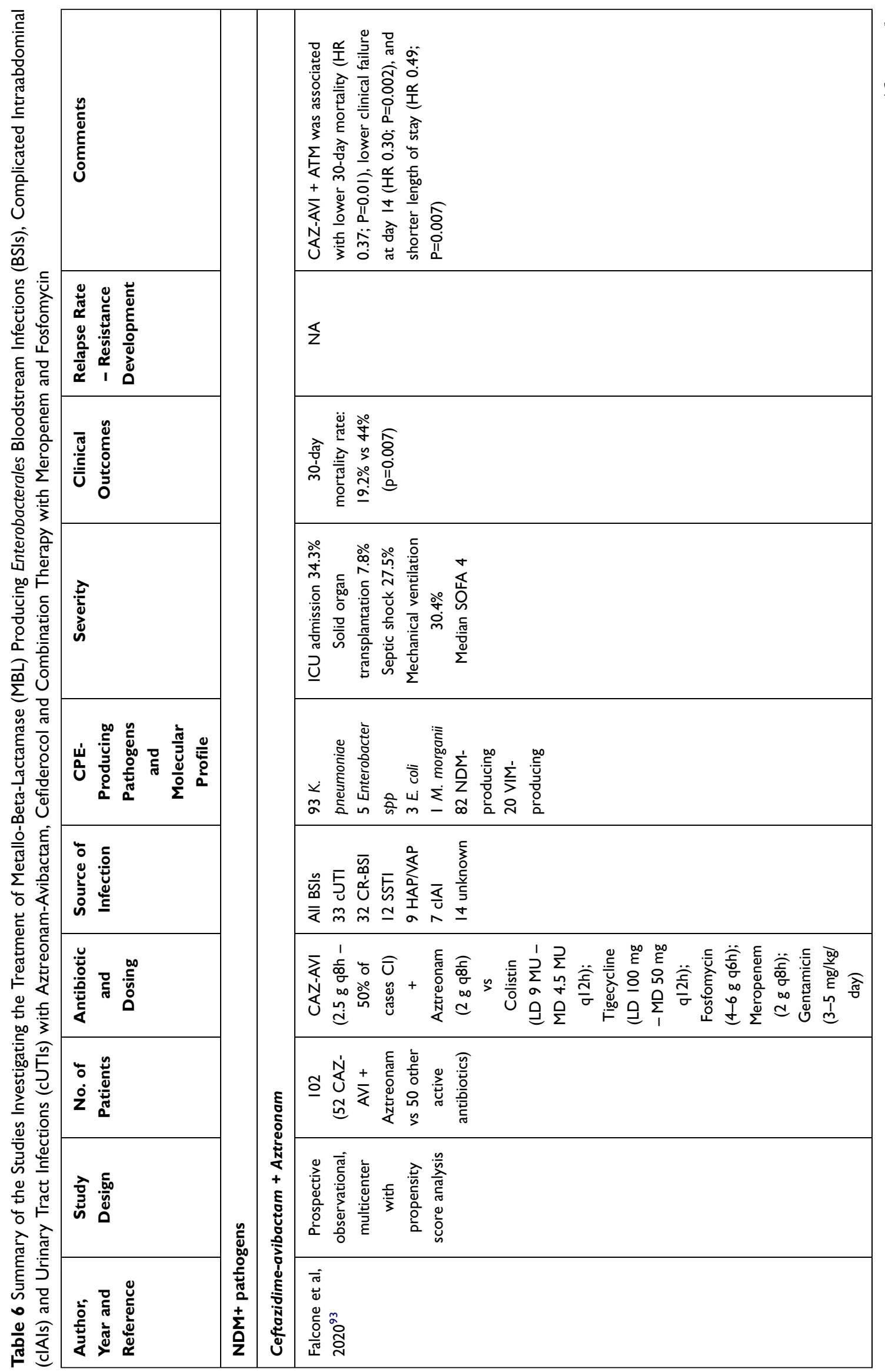




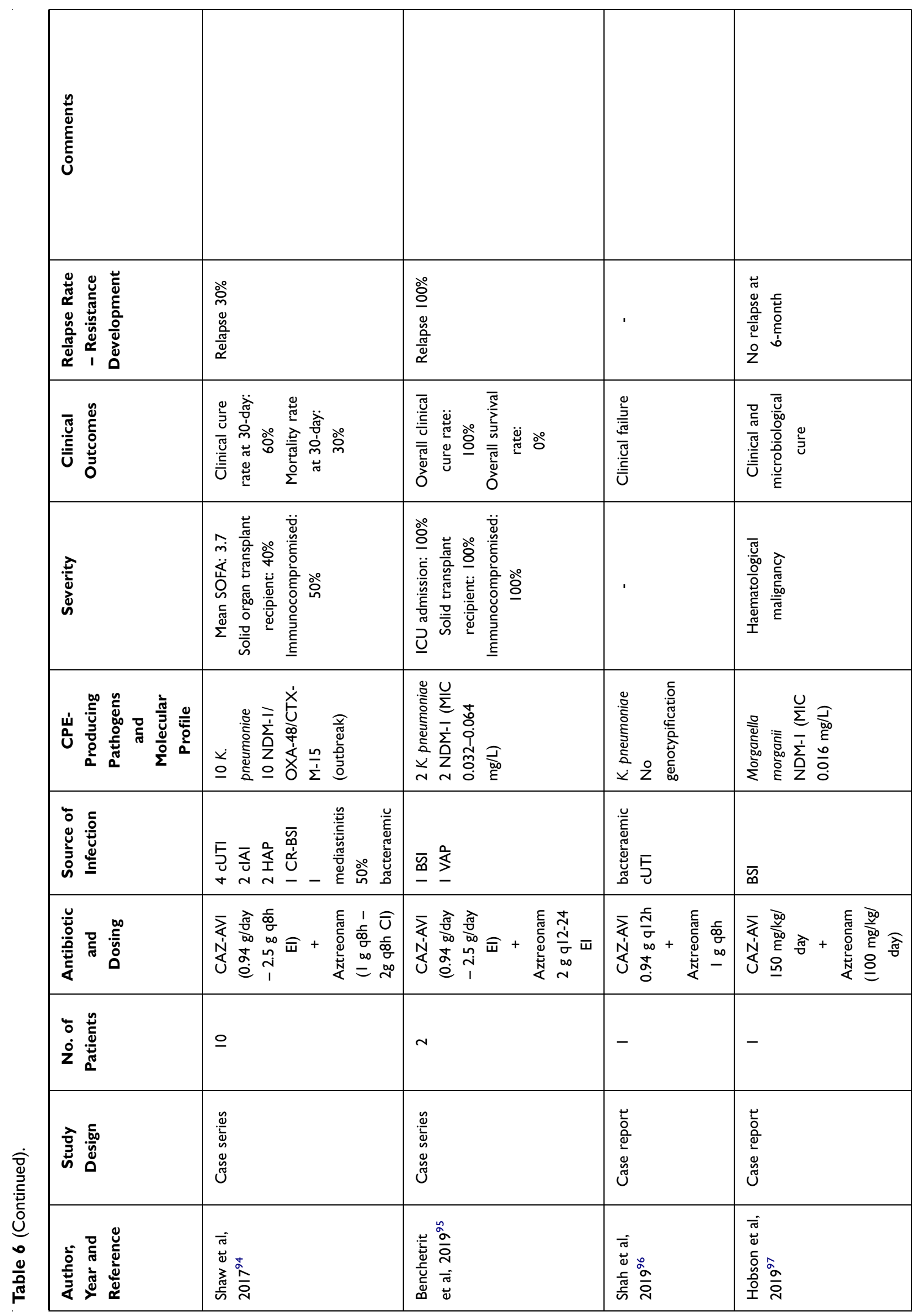




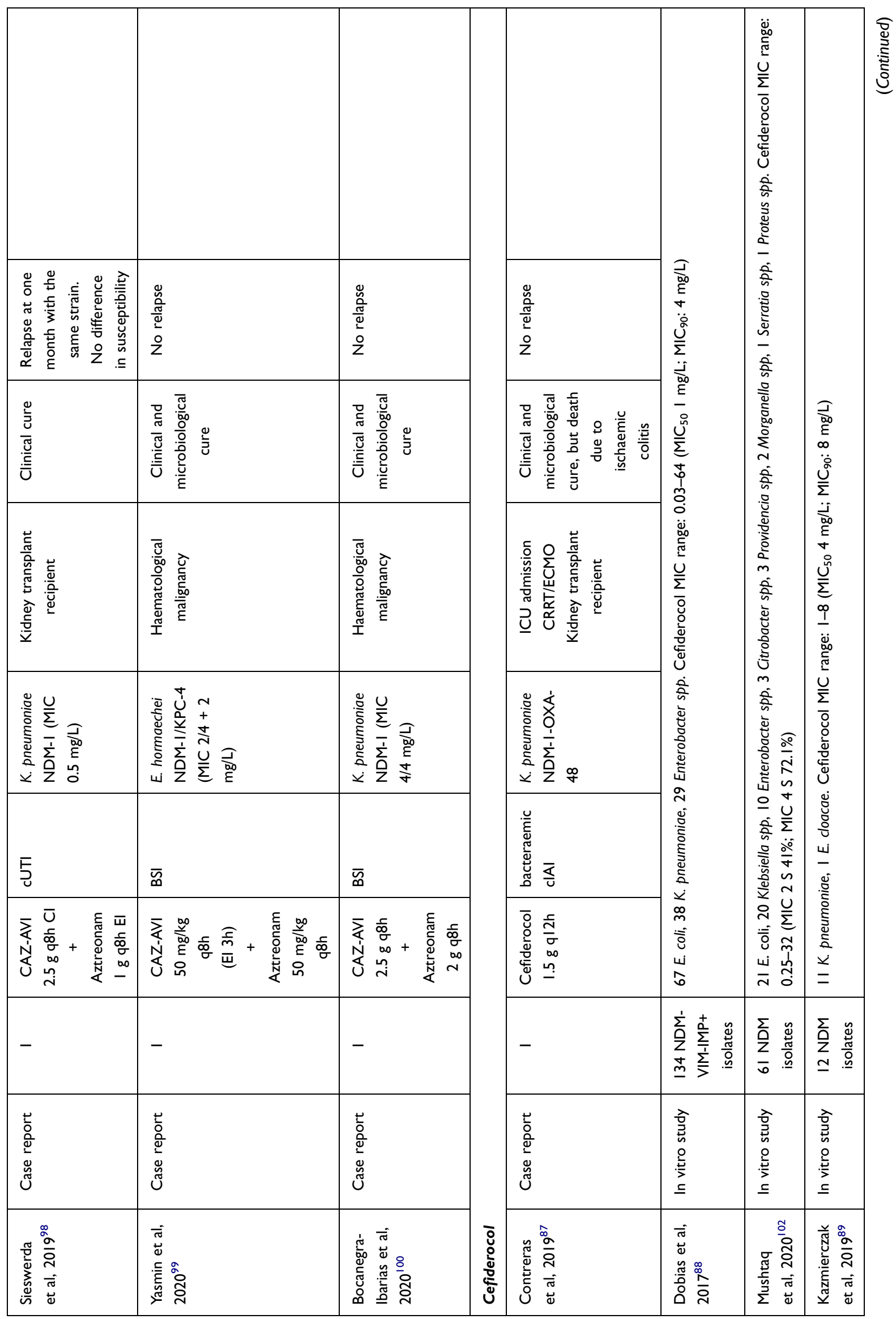




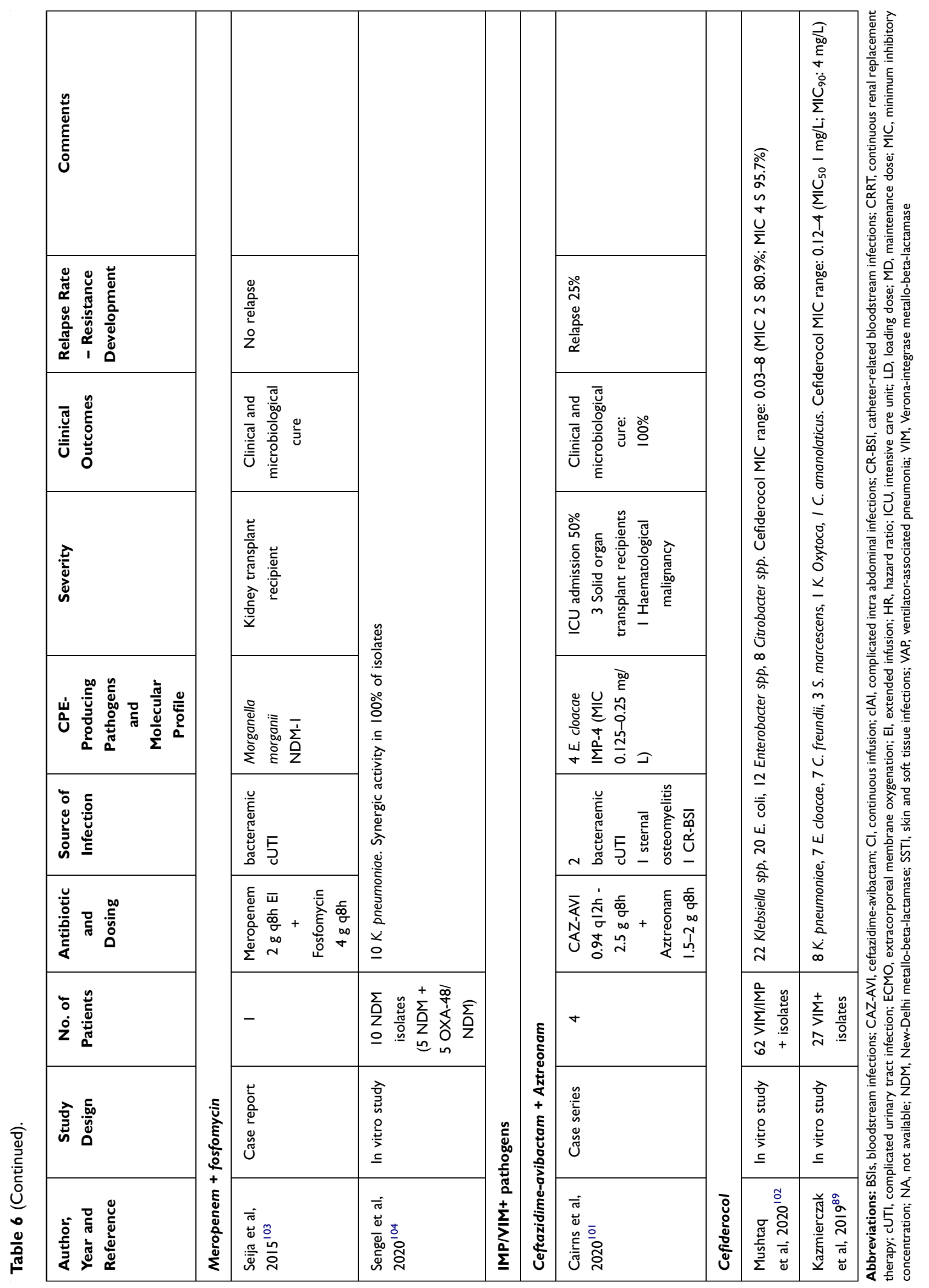


Enterobacterales with an MIC $\leq 1 \mathrm{mg} / \mathrm{L} .{ }^{108}$ Hopefully, some ongoing studies (namely MERINO-2, MERINO-3, PETERPEN, and FOREST studies) could better clarify the role of old and novel BL/BLIs in the management of critically ill patients affected by ESBL-producing Enterobacterales infections. Ceftazidime-avibactam, meropenem-vaborbactam, imipenem-relebactam, and cefiderocol may represent first-line choices for the management of critically ill patients affected by CRE infections expressing class A (eg, KPC) or D (eg, OXA-48) beta-lactamases. ${ }^{76}$ These agents should be administered in prolonged or continuous infusion as well to maximize efficacy and clinical outcome. $^{75,109}$ Monotherapy with novel BL and BL/BLIs should always be pursued in these settings considering that comparative studies showed no significant advantage in efficacy of combination therapy vs monotherapy. ${ }^{110,111}$ Against MBL-producers, the use of ceftazidime-avibactam plus aztreonam may represent an effective strategy for the management of NDM-producing Enterobacterales infections, whereas cefiderocol should be reserved mainly to VIM- or IMP-producing Enterobacterales infections. ${ }^{76}$ This latter recommendations is justified by the fact that the overall susceptibility rate of cefiderocol against NDMproducing Enterobacterales is $<70 \%$, and $\mathrm{MIC}_{50} / \mathrm{MIC}_{90}$ are 2-4-fold higher compared to VIM- or IMP-producing strains. $^{89,102,112,113}$ Fosfomycin combined with high-dose meropenem could represent a valuable alternative against both types of MBL-producing Enterobacterales infections.

Overall, altered dosing strategies of beta-lactams based on CI administration are strongly recommended for attaining very aggressive $\mathrm{PK} / \mathrm{PD}$ target of $100 \% \mathrm{fT}_{>4-8 \mathrm{xMIC}} .^{51,105}$ This approach may both maximize clinical efficacy and prevent the development of resistance. ${ }^{105} \mathrm{CI}$ is feasible with a unique daily solution infused over 24 hours for those drugs that are stable in aqueous solution at room temperature for $\geq 1$ day (eg, piperacillin-tazobactam, ceftolozane-tazobactam). Otherwise, for those drugs that are stable in aqueous solution at room temperature for 6-12h, CI may be granted through reconstitution of the aqueous solution every 6-8h and infusion over 6-8h (eg, meropenem, ampicillin/sulbactam, ceftazidime/avibactam).

Clinicians must be aware that the antibiotic dosing regimens that we recommended throughout the manuscript are focused only on treatment of patients with normal renal function. It should not be overlooked that the pharmacokinetics of hydrophilic antimicrobial agents, namely betalactams and fosfomycin, may be affected among critically ill patients by several pathophysiological conditions that may alter volume of distribution and/or renal clearance. Consequently, dose adjustments are needed in critically ill renal patients, especially among those with transient acute kidney injury, augmented renal clearance, and/or undergoing renal replacement therapy. ${ }^{114}$ Finally, it should be mentioned that for the treatment of patients with well-documented life-threatening beta-lactam allergies, alternative agents s should be considered. Fluoroquinolones, aminoglycosides and colistin could be helpful in these cases depending on the susceptibility pattern of the isolated Enterobacterales.

\section{Conclusions}

In an era characterized by the widespread diffusion of MDR Gram-negative pathogens and by the incremental spread of antibiotic resistance, implementation of a multidisciplinary approach focused at targeted therapy in critically ill patients has become a real necessity. This could simultaneously allow to promptly revise inappropriate/ unnecessary antibiotic regimens, to implement "carbapenem-sparing" strategies based on monotherapy with traditional and/or novel beta-lactams and, whenever applicable, to optimize antibiotic exposure in each single patient by means of real-time TDM guided approach. It is expected that these strategies could be helpful either in improving clinical outcome or in containing the spread of antimicrobial resistance in the ICU setting. It should be noted that the availability of rapid diagnostic technologies, based on molecular methods, that can reveal the presence of clinically-relevant resistance determinants such as the main ESBL and carbapenemase genes can be very useful to shorten the time for revision of empiric therapy according to the proposed algorithms.

\section{Disclosure}

B. Viaggi participated in advisory boards and in speaker's bureau for, and received research contracts, contributions and study events from Abbott, Accelerate Diagnostics, Ada, Alifax, Angelini, Becton Dickinson, Bellco, Biomerieux, Biotest, Cepheid, Correvio, Gilead, Menarini, MSD Italia, Nordic Pharma, Pfizer, Shionogi, Thermo Fisher Scientific; G.M. Rossolini participated in advisory boards and speaker's bureau for, and received research contracts, contributions and travel grants from Accelerate, Angelini, Arrow, Beckman Biomedical Service, Coulter, Becton-Dickinson, bioMérieux, Cepheid, Hain Life Sciences, Menarini, Meridian, MSD, Nordic Pharma, Pfizer, Qiagen, Q-linea, Qpex, Quidel, Qvella, Roche, Seegene, Set-Lance, 
Shionogi, Symcel, ThermoFisher, VenatorX, Zambon; F. Pea participated in speaker bureau for Angelini, Basilea Pharmaceutica, Gilead, Hikma, Merck Sharp \& Dohme, Nordic Pharma, Pfizer, and Sanofi Aventis, and in advisory board for Angelini, Basilea Pharmaceutica, Correvio, Gilead, Merck Sharp \& Dohme, Nordic Pharma, Novartis, Pfizer, and Thermo-Fisher. P. Viale has served as a consultant for Biomerieux, Gilead, Merck Sharp \& Dohme, Nabriva, Nordic Pharma, Pfizer, Thermo-Fisher, and Venatorx, and received payment for serving on the speaker's bureau for Correvio, Gilead, Merck Sharp \& Dohme, Nordic Pharma and Pfizer. The authors report no other conflicts of interest in this work.

\section{References}

1. Angus DC, van der Poll T. Severe sepsis and septic shock. N Engl J Med. 2013;369(9):840-851. doi:10.1056/NEJMra1208623

2. Kaukonen K-M, Bailey M, Suzuki S, Pilcher D, Bellomo R. Mortality related to severe sepsis and septic shock among critically ill patients in Australia and New Zealand, 2000-2012. JAMA. 2014;311(13):1308-1316. doi:10.1001/jama.2014.2637

3. Vincent J-L, Sakr Y, Singer M, et al. Prevalence and outcomes of infection among patients in intensive care units in 2017. JAMA. 2020;323(15):1478-1487. doi:10.1001/jama.2020.2717

4. Alberti C, Brun-Buisson C, Burchardi H, et al. Epidemiology of sepsis and infection in ICU patients from an international multicentre cohort study. Intensive Care Med. 2002;28(2):108-121. doi:10.1007/s00134-001-1143-z

5. MacVane SH. Antimicrobial resistance in the intensive care unit: a focus on gram-negative bacterial infections. $J$ Intensive Care Med. 2017;32(1):25-37. doi:10.1177/0885066615619895

6. Sader HS, Farrell DJ, Flamm RK, Jones RN. Antimicrobial susceptibility of Gram-negative organisms isolated from patients hospitalized in intensive care units in United States and European hospitals (2009-2011). Diagn Microbiol Infect Dis. 2014;78 (4):443-448. doi:10.1016/j.diagmicrobio.2013.11.025

7. Timsit J-F, Bassetti M, Cremer O, et al. Rationalizing antimicrobial therapy in the ICU: a narrative review. Intensive Care Med. 2019;45(2):172-189. doi:10.1007/s00134-019-05520-5

8. Detsis M, Karanika S, Mylonakis E. ICU acquisition rate, risk factors, and clinical significance of digestive tract colonization with extended-spectrum beta-lactamase-producing enterobacteriaceae: a systematic review and meta-analysis. Crit Care Med. 2017;45(4):705-714. doi:10.1097/CCM.0000000000002253

9. Thabit AK, Crandon JL, Nicolau DP. Antimicrobial resistance: impact on clinical and economic outcomes and the need for new antimicrobials. Expert Opin Pharmacother. 2015;16(2):159-177. doi:10.1517/14656566.2015.993381

10. Kumar A, Ellis P, Arabi Y, et al. Initiation of inappropriate antimicrobial therapy results in a fivefold reduction of survival in human septic shock. Chest. 2009;136(5):1237-1248. doi:10.1378/chest.09-0087

11. Ulldemolins M, Nuvials X, Palomar M, Masclans JR, Rello J. Appropriateness is critical. Crit Care Clin. 2011;27(1):35-51. doi:10.1016/j.ccc.2010.09.007

12. Rhodes A, Evans LE, Alhazzani W, et al. Surviving Sepsis campaign: international guidelines for management of sepsis and septic shock: 2016. Intensive Care Med. 2017;43(3):304377. doi:10.1007/s00134-017-4683-6
13. Pea F, Viale P. Bench-to-bedside review: appropriate antibiotic therapy in severe sepsis and septic shock-does the dose matter? Crit Care. 2009;13(3):214. doi:10.1186/cc7774

14. Viale P, Tedeschi S, Scudeller L, et al. Infectious Diseases Team for the Early Management of Severe Sepsis and Septic Shock in the Emergency Department. Clin Infect Dis. 2017;65(8):12531259. doi:10.1093/cid/cix548

15. Gatti M, Gasparini LE, Laratta M, et al. Intensive multidisciplinary management in critical care patients affected by severe necrotizing soft tissue infections: a cooperative method to improve the efficacy of treatment. Eur J Clin Microbiol Infect Dis. 2019;38 (6):1153-1162. doi:10.1007/s10096-019-03521-2

16. Chen $M$, Zhang $M$, Huang $P$, et al. Novel $\beta$-lactam $/ \beta$-lactamase inhibitors versus alternative antibiotics for the treatment of complicated intra-abdominal infection and complicated urinary tract infection: a meta-analysis of randomized controlled trials. Expert Rev Anti Infect Ther. 2018;16(2):111-120. doi:10.1080/ 14787210.2018.1429912

17. Gudiol C, Cuervo G, Carratalà J. Optimizing therapy of bloodstream infection due to extended-spectrum $\beta$-lactamase-producing Enterobacteriaceae. Curr Opin Crit Care. 2019;25(5):438-448. doi:10.1097/MCC.0000000000000646

18. Delgado-Valverde M, Torres E, Valiente-Mendez A, et al. Impact of the MIC of piperacillin/tazobactam on the outcome for patients with bacteraemia due to Enterobacteriaceae: the BacteraemiaMIC project. J Antimicrob Chemother. 2016;71(2):521-530. doi: $10.1093 / \mathrm{jac} / \mathrm{dkv} 362$

19. Murad MH, Asi N, Alsawas M, Alahdab F. New evidence pyramid. Evid Based Med. 2016;21(4):125-127. doi:10.1136/ebmed2016-110401

20. Eckmann C, Dryden M, Montravers P, Kozlov R, Sganga G. Antimicrobial treatment of "complicated" intra-abdominal infections and the new IDSA guidelines? a commentary and an alternative European approach according to clinical definitions. Eur J Med Res. 2011;16(3):115-126. doi:10.1186/2047783x-16-3-115

21. Solomkin JS, Mazuski JE, Bradley JS, et al. Diagnosis and management of complicated intra-abdominal infection in adults and children: guidelines by the Surgical Infection Society and the Infectious Diseases Society of America. Clin Infect Dis. 2010;50 (2):133-164. doi:10.1086/649554

22. Walker AP, Nichols RL, Wilson RF, et al. Efficacy of a betalactamase inhibitor combination for serious intraabdominal infections. Ann Surg. 1993;217(2):115-121. doi:10.1097/00000658199302000-00004

23. McKinnon PS, Neuhauser MM. Efficacy and cost of ampicillin-sulbactam and ticarcillin-clavulanate in the treatment of hospitalized patients with bacterial infections. Pharmacotherapy. 1999;19(6):724-733. doi:10.1592/phco.19.9. 724.31537

24. Rubinstein E, Lode H, Grassi C. Ceftazidime monotherapy vs. ceftriaxone/tobramycin for serious hospital-acquired gram-negative infections. Antibiotic Study Group. Clin Infect Dis. 1995;20 (5):1217-1228. doi:10.1093/clinids/20.5.1217

25. Tomera KM, Burdmann EA, Reyna OGP, et al. Ertapenem versus ceftriaxone followed by appropriate oral therapy for treatment of complicated urinary tract infections in adults: results of a prospective, randomized, double-blind multicenter study. Antimicrob Agents Chemother. 2002;46(9):2895-2900. doi:10.1128/aac.46. 9.2895-2900.2002

26. Jimenez-Cruz F, Jasovich A, Cajigas J, et al. A prospective, multicenter, randomized, double-blind study comparing ertapenem and ceftriaxone followed by appropriate oral therapy for complicated urinary tract infections in adults. Urology. 2002;60 (1):16-22. doi:10.1016/s0090-4295(02)01664-3 
27. Wells WG, Woods GL, Jiang Q, Gesser RM. Treatment of complicated urinary tract infection in adults: combined analysis of two randomized, double-blind, multicentre trials comparing ertapenem and ceftriaxone followed by appropriate oral therapy. $J$ Antimicrob Chemother. 2004;53(Suppl 2):ii67-74. doi:10.1093/ jac/dkh208

28. Park DW, Peck KR, Chung MH, et al. Comparison of ertapenem and ceftriaxone therapy for acute pyelonephritis and other complicated urinary tract infections in Korean adults: a randomized, double-blind, multicenter trial. J Korean Med Sci. 2012;27 (5):476-483. doi:10.3346/jkms.2012.27.5.476

29. Paterson DL, Henderson A, Harris PNA. Current evidence for therapy of ceftriaxone-resistant Gram-negative bacteremia. Curr Opin Infect Dis. 2020;33(1):78-85. doi:10.1097/QCO.0000000000000623

30. Papadimitriou-Olivgeris M, Bartzavali C, Lambropoulou A, et al. Reversal of carbapenemase-producing Klebsiella pneumoniae epidemiology from blaKPC- to blaVIM-harbouring isolates in a Greek ICU after introduction of ceftazidime/avibactam. J Antimicrob Chemother. 2019;74(7):2051-2054. doi:10.1093/jac/dkz125

31. Harris PNA, Tambyah PA, Lye DC, et al. Effect of Piperacillintazobactam vs meropenem on 30-day mortality for patients with $\mathrm{E}$ coli or Klebsiella pneumoniae bloodstream infection and ceftriaxone resistance: a randomized clinical trial. JAMA. 2018;320 (10):984-994. doi:10.1001/jama.2018.12163

32. Rodríguez-Baño J, Gutiérrez-Gutiérrez B, Kahlmeter G. Antibiotics for Ceftriaxone-resistant gram-negative bacterial bloodstream infections. JAMA. 2019;321(6):612-613. doi:10.1001/jama.2018.19345

33. Livermore DM, Day M, Cleary P, et al. OXA-1 $\beta$-lactamase and non-susceptibility to penicillin/ $\beta$-lactamase inhibitor combinations among ESBL-producing Escherichia coli. J Antimicrob Chemother. 2019;74(2):326-333. doi:10.1093/jac/dky453

34. Sfeir MM, Askin G, Christos P. Beta-lactam/beta-lactamase inhibitors versus carbapenem for bloodstream infections due to extended-spectrum beta-lactamase-producing Enterobacteriaceae: systematic review and meta-analysis. Int $J$ Antimicrob Agents. 2018;52(5):554-570. doi:10.1016/j.ijantimicag.2018.07.021

35. Rodríguez-Baño J, Navarro MD, Retamar P, Picón E, Á P. Extended-Spectrum Beta-Lactamases-Red Española de Investigación en Patología Infecciosa/Grupo de Estudio de Infección Hospitalaria Group. $\beta$-Lactam $/ \beta$-lactam inhibitor combinations for the treatment of bacteremia due to extended-spectrum $\beta$-lactamase-producing Escherichia coli: a post hoc analysis of prospective cohorts. Clin Infect Dis. 2012;54(2):167-174. doi:10.1093/cid/cir790

36. Kang C-I, Park SY, Chung DR, Peck KR, Song J-H. Piperacillintazobactam as an initial empirical therapy of bacteremia caused by extended-spectrum $\beta$-lactamase-producing Escherichia coli and Klebsiella pneumoniae. $J$ Infect. 2012;64(5):533-534 doi:10.1016/j.jinf.2012.01.008

37. Harris PNA, Yin M, Jureen R, et al. Comparable outcomes for $\beta$ lactam/ $\beta$-lactamase inhibitor combinations and carbapenems in definitive treatment of bloodstream infections caused by cefotaxime-resistant Escherichia coli or Klebsiella pneumoniae. Antimicrob Resist Infect Control. 2015;4:14. doi:10.1186/ s13756-015-0055-6

38. Gutiérrez-Gutiérrez B, Pérez-Galera S, Salamanca E, et al. A multinational, preregistered cohort study of $\beta$-Lactam $/ \beta$-lactamase inhibitor combinations for treatment of bloodstream infections due to extended-spectrum- $\beta$-lactamase-producing enterobacteriaceae. Antimicrob Agents Chemother. 2016;60(7):4159-4169. doi:10.1128/AAC.00365-16

39. Ng TM, Khong WX, Harris PNA, et al. Empiric Piperacillintazobactam versus carbapenems in the treatment of bacteraemia due to extended-spectrum beta-lactamase-producing enterobacteriaceae. PLoS One. 2016;11(4):e0153696. doi:10.1371/journal. pone. 0153696
40. Gudiol C, Royo-Cebrecos C, Abdala E, et al. Efficacy of $\beta$ Lactam/ $\beta$-lactamase inhibitor combinations for the treatment of bloodstream infection due to extended-spectrum- $\beta$-lactamase-producing enterobacteriaceae in hematological patients with neutropenia. Antimicrob Agents Chemother. 2017;61:8. doi:10.1128/ AAC.00164-17

41. Yoon YK, Kim JH, Sohn JW, Yang KS, Kim MJ. Role of piperacillin/tazobactam as a carbapenem-sparing antibiotic for treatment of acute pyelonephritis due to extended-spectrum $\beta$ lactamase-producing Escherichia coli. Int $J$ Antimicrob Agents. 2017;49(4):410-415. doi:10.1016/j.ijantimicag.2016.12.017

42. Ko J-H, Lee NR, Joo E-J, et al. Appropriate non-carbapenems are not inferior to carbapenems as initial empirical therapy for bacteremia caused by extended-spectrum beta-lactamase-producing Enterobacteriaceae: a propensity score weighted multicenter cohort study. Eur J Clin Microbiol Infect Dis. 2018;37(2):305311. doi:10.1007/s10096-017-3133-2

43. Benanti GE, Brown ART, Shigle TL, et al. Carbapenem versus cefepime or piperacillin-tazobactam for empiric treatment of bacteremia due to extended-spectrum- $\beta$-lactamase-producing Escherichia coli in patients with hematologic malignancy. Antimicrob Agents Chemother. 2019;63:2. doi:10.1128/AAC.01813-18

44. John R, Colley P, Nguyen HL, Berhe M. Outcomes analysis in patients with extended-spectrum beta-lactamase bacteremia empirically treated with piperacillin/tazobactam versus carbapenems. Proc (Bayl Univ Med Cent). 2019;32(2):187-191. doi:10.1080/08998280.2019.1582466

45. Nasir N, Ahmed S, Razi S, Awan S, Mahmood SF. Risk factors for mortality of patients with ceftriaxone resistant E. coli bacteremia receiving carbapenem versus beta lactam/beta lactamase inhibitor therapy. BMC Res Notes. 2019;12(1):611. doi:10.1186/ s13104-019-4648-7

46. Sharara SL, Amoah J, Pana ZD, Simner PJ, Cosgrove SE, Tamma PD. Is Piperacillin-Tazobactam Effective for the Treatment of Pyelonephritis Caused by Extended-Spectrum $\beta$-LactamaseProducing Organisms? Clin Infect Dis. 2020;71(8):e331-e337. doi:10.1093/cid/ciz1205

47. Seo YB, Lee J, Kim YK, et al. Randomized controlled trial of piperacillin-tazobactam, cefepime and ertapenem for the treatment of urinary tract infection caused by extended-spectrum beta-lactamase-producing Escherichia coli. BMC Infect Dis. 2017;17(1):404. doi:10.1186/s12879-017-2502-x

48. Tamma PD, Han JH, Rock C, et al. Carbapenem therapy is associated with improved survival compared with piperacillin-tazobactam for patients with extended-spectrum $\beta$-lactamase bacteremia. Clin Infect Dis. 2015;60(9):1319-1325. doi:10.1093/cid/civ003

49. Ofer-Friedman H, Shefler C, Sharma S, et al. Carbapenems versus piperacillin-tazobactam for bloodstream infections of nonurinary source caused by extended-spectrum beta-lactamase-producing enterobacteriaceae. Infect Control Hosp Epidemiol. 2015;36 (8):981-985. doi:10.1017/ice.2015.101

50. Vardakas KZ, Voulgaris GL, Maliaros A, Samonis G, Falagas ME. Prolonged versus short-term intravenous infusion of antipseudomonal $\beta$-lactams for patients with sepsis: a systematic review and meta-analysis of randomised trials. Lancet Infect Dis. 2018;18(1):108-120. doi:10.1016/S1473-3099(17)30615-1

51. Thabit AK, Hobbs ALV, Guzman OE, Shea KM. the pharmacodynamics of prolonged infusion $\beta$-lactams for the treatment of pseudomonas aeruginosa infections: a systematic review. Clin Ther. 2019;41 (11):2397-2415.e8. doi:10.1016/j.clinthera.2019.09.010

52. Wagenlehner FM, Umeh O, Steenbergen J, Yuan G, Darouiche RO. Ceftolozane-tazobactam compared with levofloxacin in the treatment of complicated urinary-tract infections, including pyelonephritis: a randomised, double-blind, Phase 3 trial (ASPECTcUTI). Lancet. 2015;385(9981):1949-1956. doi:10.1016/S01406736(14)62220-0 
53. Solomkin J, Hershberger E, Miller B, et al. Ceftolozane/ Tazobactam plus metronidazole for complicated intra-abdominal infections in an era of multidrug resistance: results from a randomized, double-blind, phase 3 trial (ASPECT-cIAI). Clin Infect Dis. 2015;60(10):1462-1471. doi:10.1093/cid/civ097

54. Wagenlehner FM, Sobel JD, Newell P, et al. Ceftazidime-avibactam Versus doripenem for the treatment of complicated urinary tract infections, including acute pyelonephritis: RECAPTURE, a Phase 3 randomized trial program. Clin Infect Dis. 2016;63 (6):754-762. doi:10.1093/cid/ciw378

55. Mazuski JE, Gasink LB, Armstrong J, et al. Efficacy and safety of ceftazidime-avibactam plus metronidazole versus meropenem in the treatment of complicated intra-abdominal infection: results from a randomized, controlled, double-blind, phase 3 program. Clin Infect Dis. 2016;62(11):1380-1389. doi:10.1093/cid/ciw133

56. Qin X, Tran BG, Kim MJ, et al. A randomised, double-blind, phase 3 study comparing the efficacy and safety of ceftazidime/avibactam plus metronidazole versus meropenem for complicated intra-abdominal infections in hospitalised adults in Asia. Int $J$ Antimicrob Agents. 2017;49(5):579-588. doi:10.1016/j.ijantimicag.2017.01.010

57. Carmeli Y, Armstrong J, Laud PJ, et al. Ceftazidime-avibactam or best available therapy in patients with ceftazidime-resistant Enterobacteriaceae and Pseudomonas aeruginosa complicated urinary tract infections or complicated intra-abdominal infections (REPRISE): a randomised, pathogen-directed, phase 3 study. Lancet Infect Dis. 2016;16(6):661-673. doi:10.1016/S1473-3099(16)30004-4

58. Meini S, Tascini C, Cei M, Sozio E, Rossolini GM. AmpC $\beta$ lactamase-producing Enterobacterales: what a clinician should know. Infection. 2019;47(3):363-375. doi:10.1007/s15010-01901291-9

59. Harris PNA, Wei JY, Shen AW, et al. Carbapenems versus alternative antibiotics for the treatment of bloodstream infections caused by Enterobacter, Citrobacter or Serratia species: a systematic review with meta-analysis. J Antimicrob Chemother. 2016;71(2):296-306. doi:10.1093/jac/dkv346

60. Blanchette LM, Kuti JL, Nicolau DP, Nailor MD. Clinical comparison of ertapenem and cefepime for treatment of infections caused by AmpC beta-lactamase-producing Enterobacteriaceae. Scand J Infect Dis. 2014;46(11):803-808. doi:10.3109/ 00365548.2014.954262

61. Tamma PD, Girdwood SCT, Gopaul R, et al. The use of cefepime for treating AmpC $\beta$-lactamase-producing Enterobacteriaceae. Clin Infect Dis. 2013;57(6):781-788. doi:10.1093/cid/cit395

62. Siedner MJ, Galar A, Guzmán-Suarez BB, et al. Cefepime vs other antibacterial agents for the treatment of Enterobacter species bacteremia. Clin Infect Dis. 2014;58(11):1554-1563. doi:10. 1093/cid/ciu182

63. Hilty M, Sendi P, Seiffert SN, et al. Characterisation and clinical features of Enterobacter cloacae bloodstream infections occurring at a tertiary care university hospital in Switzerland: is cefepime adequate therapy? Int J Antimicrob Agents. 2013;41(3):236-249. doi:10.1016/j.ijantimicag.2012.10.022

64. Tan SH, Ng TM, Chew KL, et al. Outcomes of treating AmpCproducing Enterobacterales bacteraemia with carbapenems vs. non-carbapenems. Int J Antimicrob Agents. 2020;55(2):105860. doi:10.1016/j.ijantimicag.2019.105860

65. Lee N-Y, Lee -C-C, Li C-W, et al. Cefepime therapy for monomicrobial enterobacter cloacae bacteremia: unfavorable outcomes in patients infected by cefepime-susceptible dose-dependent isolates. Antimicrob Agents Chemother. 2015;59(12):7558-7563. doi:10.1128/AAC.01477-15

66. Cheng L, Nelson BC, Mehta M, et al. Piperacillin-tazobactam versus other antibacterial agents for treatment of bloodstream infections due to AmpC $\beta$-lactamase-producing enterobacteriaceae. Antimicrob Agents Chemother. 2017;61:6. doi:10.1128/ AAC.00276-17
67. van Duin D, Lok JJ, Earley M, et al. Colistin versus ceftazidimeavibactam in the treatment of infections due to carbapenem-resistant enterobacteriaceae. Clin Infect Dis. 2018;66(2):163-171. doi: $10.1093 / \mathrm{cid} / \mathrm{cix} 783$

68. Castón JJ, Lacort-Peralta I, Martín-Dávila P, et al. Clinical efficacy of ceftazidime/avibactam versus other active agents for the treatment of bacteremia due to carbapenemase-producing Enterobacteriaceae in hematologic patients. Int $J$ Infect Dis. 2017;59:118-123. doi:10.1016/j.ijid.2017.03.021

69. Tumbarello M, Trecarichi EM, Corona A, et al. Efficacy of ceftazidime-avibactam salvage therapy in patients with infections caused by klebsiella pneumoniae carbapenemase-producing $\mathrm{K}$. pneumoniae. Clin Infect Dis. 2019;68(3):355-364. doi:10.1093/ cid/ciy492

70. Shields RK, Nguyen MH, Chen L, et al. Ceftazidime-Avibactam is superior to other treatment regimens against carbapenem-resistant klebsiella pneumoniae bacteremia. Antimicrob Agents Chemother. 2017;61:8. doi:10.1128/AAC.00883-17

71. Temkin E, Torre-Cisneros J, Beovic B, et al. CeftazidimeAvibactam as salvage therapy for infections caused by carbapenem-resistant organisms. Antimicrob Agents Chemother. 2017;61:2. doi:10.1128/AAC.01964-16

72. King M, Heil E, Kuriakose S, et al. Multicenter study of outcomes with ceftazidime-avibactam in patients with carbapenemresistant enterobacteriaceae infections. Antimicrob Agents Chemother. 2017;61:7. doi:10.1128/AAC.00449-17

73. Shields RK, Potoski BA, Haidar G, et al. Clinical outcomes, drug toxicity, and emergence of ceftazidime-avibactam resistance among patients treated for carbapenem-resistant enterobacteriaceae infections. Clin Infect Dis. 2016;63(12):1615-1618. doi:10.1093/cid/ciw636

74. Shields RK, Nguyen MH, Chen L, Press EG, Kreiswirth BN, Clancy CJ. Pneumonia and renal replacement therapy are risk factors for ceftazidime-avibactam treatment failures and resistance among patients with carbapenem-resistant enterobacteriaceae infections. Antimicrob Agents Chemother. 2018;62:5. doi:10.1128/AAC.02497-17

75. Tumbarello M, Raffaelli F, Giannella M, et al. Ceftazidime-avibactam use for KPC-Kp infections: a retrospective observational multicenter study. Clin Infect Dis. 2021. doi:10.1093/cid/ciab176

76. Yahav D, Giske CG, Grāmatniece A, Abodakpi H, Tam VH, New LL. $\beta$-Lactam- $\beta$-Lactamase Inhibitor Combinations. Clin Microbiol Rev. 2020;34:1. doi:10.1128/CMR.00115-20

77. Wunderink RG, Giamarellos-Bourboulis EJ, Rahav G, et al. Effect and safety of meropenem-vaborbactam versus best-available therapy in patients with carbapenem-resistant enterobacteriaceae infections: the TANGO II randomized clinical trial. Infect Dis Ther. 2018;7(4):439-455. doi:10.1007/s40121-018-0214-1

78. Ackley R, Roshdy D, Meredith J, et al. Meropenem-Vaborbactam versus Ceftazidime-Avibactam for Treatment of CarbapenemResistant Enterobacteriaceae Infections. Antimicrob Agents Chemother. 2020;64:5. doi:10.1128/AAC.02313-19

79. Alosaimy S, Jorgensen SCJ, Lagnf AM, et al. Real-world multicenter analysis of clinical outcomes and safety of meropenemvaborbactam in patients treated for serious gram-negative bacterial infections. Open Forum Infect Dis. 2020;7(3):ofaa051. doi:10.1093/ofid/ofaa051

80. Shields RK, McCreary EK, Marini RV, et al. Early experience with meropenem-vaborbactam for treatment of carbapenem-resistant enterobacteriaceae infections. Clin Infect Dis. 2020;71 (3):667-671. doi:10.1093/cid/ciz1131

81. Motsch J, Murta de Oliveira C, Stus V, et al. RESTORE-IMI 1: a Multicenter, Randomized, Double-blind Trial Comparing Efficacy and Safety of Imipenem/Relebactam vs Colistin Plus Imipenem in Patients With Imipenem-nonsusceptible Bacterial Infections. Clin Infect Dis. 2020;70(9):1799-1808. doi:10.1093/cid/ciz530 
82. Bassetti M, Echols R, Matsunaga Y, et al. Efficacy and safety of cefiderocol or best available therapy for the treatment of serious infections caused by carbapenem-resistant Gram-negative bacteria (CREDIBLE-CR): a randomised, open-label, multicentre, pathogen-focused, descriptive, phase 3 trial. Lancet Infect Dis. 2021;21(2):226-240. doi:10.1016/S1473-3099(20)30796-9

83. Stewart A, Harris P, Henderson A, Paterson D. Treatment of Infections by OXA-48-Producing Enterobacteriaceae. Antimicrob Agents Chemother. 2018;62:11. doi:10.1128/ AAC.01195-18

84. Alraddadi BM, Saeedi M, Qutub M, Alshukairi A, Hassanien A, Wali G. Efficacy of ceftazidime-avibactam in the treatment of infections due to Carbapenem-resistant Enterobacteriaceae. BMC Infect Dis. 2019;19(1):772. doi:10.1186/s12879-019-4409-1

85. De la Calle C, Rodríguez O, Morata L, et al. Clinical characteristics and prognosis of infections caused by OXA-48 carbapenemase-producing Enterobacteriaceae in patients treated with ceftazidime-avibactam. Int $J$ Antimicrob Agents. 2019;53 (4):520-524. doi:10.1016/j.ijantimicag.2018.11.015

86. Sousa A, Pérez-Rodríguez MT, Soto A, et al. Effectiveness of ceftazidime/avibactam as salvage therapy for treatment of infections due to OXA-48 carbapenemase-producing Enterobacteriaceae. J Antimicrob Chemother. 2018;73(11):31703175. doi: $10.1093 /$ jac/dky295

87. Contreras DA, Fitzwater SP, Nanayakkara DD, et al. Coinfections of Two Strains of NDM-1- and OXA-232-Coproducing Klebsiella pneumoniae in a Kidney Transplant Patient. Antimicrob Agents Chemother. 2020;64:4. doi:10.1128/AAC.00948-19

88. Dobias J, Dénervaud-Tendon V, Poirel L, Nordmann P. Activity of the novel siderophore cephalosporin cefiderocol against multidrug-resistant Gram-negative pathogens. Eur J Clin Microbiol Infect Dis. 2017;36(12):2319-2327. doi:10.1007/s10096-0173063-z

89. Kazmierczak KM, Tsuji M, Wise MG, et al. In vitro activity of cefiderocol, a siderophore cephalosporin, against a recent collection of clinically relevant carbapenem-non-susceptible Gramnegative bacilli, including serine carbapenemase- and metallo- $\beta$ lactamase-producing isolates (SIDERO-WT-2014 Study). Int $J$ Antimicrob Agents. 2019;53(2):177-184. doi:10.1016/j. ijantimicag.2018.10.007

90. Jacobs MR, Abdelhamed AM, Good CE, et al. ARGONAUT-I: activity of Cefiderocol (S-649266), a Siderophore Cephalosporin, against Gram-Negative Bacteria, Including Carbapenem-Resistant Nonfermenters and Enterobacteriaceae with Defined ExtendedSpectrum $\beta$-Lactamases and Carbapenemases. Antimicrob Agents Chemother. 2019;63:1. doi:10.1128/AAC.01801-18

91. Delgado-Valverde M, Conejo MDC, Serrano L, FernándezCuenca F, Pascual Á. Activity of cefiderocol against high-risk clones of multidrug-resistant Enterobacterales, Acinetobacter baumannii, Pseudomonas aeruginosa and Stenotrophomonas maltophilia. J Antimicrob Chemother. 2020;75(7):1840-1849. doi:10.1093/jac/dkaa117

92. Boyd SE, Livermore DM, Hooper DC, Hope WW. Metallo- $\beta$ Lactamases: structure, function, epidemiology, treatment options, and the development pipeline. Antimicrob Agents Chemother. 2020;64:10. doi:10.1128/AAC.00397-20

93. Falcone M, Daikos GL, Tiseo G, et al. Efficacy of ceftazidimeavibactam plus aztreonam in patients with bloodstream infections caused by MBL- producing Enterobacterales. Clin Infect Dis. 2020. doi:10.1093/cid/ciaa586

94. Shaw E, Rombauts A, Tubau F, et al. Clinical outcomes after combination treatment with ceftazidime/avibactam and aztreonam for NDM-1/OXA-48/CTX-M-15-producing Klebsiella pneumoniae infection. J Antimicrob Chemother. 2018;73(4):1104-1106. doi:10.1093/jac/dkx496
95. Benchetrit L, Mathy V, Armand-Lefevre L, Bouadma L, Timsit JF. Successful treatment of septic shock due to NDM-1-producing Klebsiella pneumoniae using ceftazidime/avibactam combined with aztreonam in solid organ transplant recipients: report of two cases. Int $J$ Antimicrob Agents. 2020;55(1):105842. doi:10.1016/j.ijantimicag.2019.10.023

96. Shah PJ, Tran T, Emelogu F, Tariq F. Aztreonam, Ceftazidime/ Avibactam, and Colistin Combination for the Management of Carbapenemase-Producing Klebsiella Pneumoniae Bacteremia: a Case Report. J Pharm Pract. 2019;897190019882262. doi:10.1177/0897190019882262

97. Hobson CA, Bonacorsi S, Fahd M, et al. Successful Treatment of Bacteremia Due to NDM-1-Producing Morganella morganii with Aztreonam and Ceftazidime-Avibactam Combination in a Pediatric Patient with Hematologic Malignancy. Antimicrob Agents Chemother. 2019;63:2. doi:10.1128/AAC.02463-18

98. Sieswerda E, van den Brand $M$, van den Berg RB, et al. Successful rescue treatment of sepsis due to a pandrug-resistant, NDM-producing Klebsiella pneumoniae using aztreonam powder for nebulizer solution as intravenous therapy in combination with ceftazidime/avibactam. $J$ Antimicrob Chemother. 2020;75 (3):773-775. doi:10.1093/jac/dkz495

99. Yasmin M, Fouts DE, Jacobs MR, et al. Monitoring CeftazidimeAvibactam and Aztreonam Concentrations in the Treatment of a Bloodstream Infection Caused by a Multidrug-Resistant Enterobacter sp. Carrying Both Klebsiella pneumoniae Carbapenemase-4 and New Delhi Metallo- $\beta$-Lactamase-1. Clin Infect Dis. 2020;71(4):1095-1098. doi:10.1093/cid/ciz1155

100. Bocanegra-Ibarias P, Camacho-Ortiz A, Garza-González E, Flores-Treviño S, Kim H, Perez-Alba E. Aztreonam plus ceftazidime-avibactam as treatment of NDM-1-producing Klebsiella pneumoniae bacteraemia in a neutropenic patient: last resort therapy? J Glob Antimicrob Resist. 2020;23:417-419. doi:10.1016/j. jgar.2020.10.019

101. Cairns KA, Hall V, Martin GE, et al. Treatment of invasive IMP-4 Enterobacter cloacae infection in transplant recipients using ceftazidime/avibactam with aztreonam: a case series and literature review. Transpl Infect Dis. 2020. doi:10.1111/tid.13510

102. Mushtaq S, Sadouki Z, Vickers A, Livermore DM, Woodford N. In Vitro Activity of Cefiderocol, a Siderophore Cephalosporin, against Multidrug-Resistant Gram-Negative Bacteria. Antimicrob Agents Chemother. 2020;64:12. doi:10.1128/AAC.01582-20

103. Seija V, Medina Presentado JC, Bado I, et al. Sepsis caused by New Delhi metallo- $\beta$-lactamase (blaNDM-1) and qnrD-producing Morganella morganii, treated successfully with fosfomycin and meropenem: case report and literature review. Int J Infect Dis. 2015;30:20-26. doi:10.1016/j.ijid.2014.09.010

104. Erturk Sengel B, Altinkanat Gelmez G, Soyletir G, Korten V. In vitro synergistic activity of fosfomycin in combination with meropenem, amikacin and colistin against OXA-48 and/or NDMproducing Klebsiella pneumoniae. $J$ Chemother. 2020;32 (5):237-243. doi:10.1080/1120009X.2020.1745501

105. Tamma PD, The R-BJ. Use of noncarbapenem $\beta$-lactams for the treatment of extended-spectrum $\beta$-lactamase infections. Clin Infect Dis. 2017;64(7):972-980. doi:10.1093/cid/cix034

106. Collins VL, Marchaim D, Pogue JM, et al. Efficacy of ertapenem for treatment of bloodstream infections caused by extended-spectrum- $\beta$-lactamase-producing Enterobacteriaceae. Antimicrob Agents Chemother. 2012;56(4):2173-2177. doi:10.1128/ AAC.05913-11

107. Gutiérrez-Gutiérrez B, Bonomo RA, Carmeli Y, et al. Ertapenem for the treatment of bloodstream infections due to ESBL-producing Enterobacteriaceae: a multinational pre-registered cohort study. J Antimicrob Chemother. 2016;71(6):1672-1680. doi:10. 1093/jac/dkv502 
108. Karaiskos I, Carbapenem-Sparing GH. Strategies for ESBL producers: when and how. Antibiotics (Basel). 2020;9:2. doi:10.3390/ antibiotics 9020061

109. Pilmis B, Petitjean G, Lesprit P, et al. Continuous infusion of ceftolozane/tazobactam is associated with a higher probability of target attainment in patients infected with Pseudomonas aeruginosa. Eur J Clin Microbiol Infect Dis. 2019;38(8):1457-1461. doi:10.1007/s10096-019-03573-4

110. Meini S, Viaggi B, Tascini C. Mono vs. combo regimens with novel beta-lactam/beta-lactamase inhibitor combinations for the treatment of infections due to carbapenemase-producing Enterobacterales: insights from the literature. Infection. 2021. doi:10.1007/s15010-021-01577-x

111. Onorato L, Di Caprio G, Signoriello S, Coppola N. Efficacy of ceftazidime/avibactam in monotherapy or combination therapy against carbapenem-resistant Gram-negative bacteria: a meta-analysis. Int J Antimicrob Agents. 2019;54(6):735-740. doi:10.1016/ j.ijantimicag.2019.08.025
112. Giacobbe DR, Ciacco E, Girmenia C, et al. Evaluating Cefiderocol in the Treatment of Multidrug-Resistant GramNegative Bacilli: a Review of the Emerging Data. Infect Drug Resist. 2020;13:4697-4711. doi:10.2147/IDR.S205309

113. Kohira N, Hackel MA, Ishioka Y, et al. Reduced susceptibility mechanism to cefiderocol, a siderophore cephalosporin, among clinical isolates from a global surveillance programme (SIDEROWT-2014). J Glob Antimicrob Resist. 2020;22:738-741. doi:10. 1016/j.jgar.2020.07.009

114. Gatti M, Pea F. Pharmacokinetic/pharmacodynamic target attainment in critically ill renal patients on antimicrobial usage: focus on novel beta-lactams and beta lactams/beta-lactamase inhibitors. Expert Rev Clin Pharmacol. 2021. doi:10.1080/17512433. 2021.1901574

\section{Publish your work in this journal}

Infection and Drug Resistance is an international, peer-reviewed openaccess journal that focuses on the optimal treatment of infection (bacterial, fungal and viral) and the development and institution of preventive strategies to minimize the development and spread of resistance. The journal is specifically concerned with the epidemiology of antibiotic resistance and the mechanisms of resistance development and diffusion in both hospitals and the community. The manuscript management system is completely online and includes a very quick and fair peerreview system, which is all easy to use. Visit http://www.dovepress.com/ testimonials.php to read real quotes from published authors. 